\title{
A taxonomic revision of Henckelia (Gesneriaceae) in South India with a new species, one new combination and seven lectotypifications
}

\author{
Janeesha A.P. ${ }^{1}$ \& S. Nampy²* \\ 'Department of Botany, Providence Women's College, Malaparamba P.O., Kozhikode, Kerala - 673 009, India. \\ 2Department of Botany, University of Calicut, Calicut University P.O., \\ Malappuram, Kerala - 673 635, India. \\ *E-mail: santhoshnampy2019@gmail.com
}

\begin{abstract}
The genus Henckelia Spreng. (Gesneriaceae) in South India is revisited based on field and herbarium data. Fifteen species are recognized. Henckelia wayanadensis is described new to science while Didymocarpus lyratus Wight var. protractus C.B.Clarke and Henckelia sivagiriensis (Rajakumar, Selvak., S.Murug. \& Chellap.) E.S.S.Kumar are reduced to synonymy of Henckelia lyrata (Wight) A.Weber \& B.L.Burtt and Henckelia wightii (C.B.Clarke) A.Weber \& B.L.Burtt respectively. Lectotypification of seven names are carried out in this paper. Detailed descriptions, illustration/ photographic images and key for the identification of South Indian species are provided.
\end{abstract}

Keywords: Conservation assessments, Endemic, $H$. wayanadensis, Lectotypifications, New combination.

\section{Introduction}

The genus Henckelia Spreng. was first recognized as Roettlera by Vahl in 1804. Since this name was preoccupied with another genus, it was renamed Henckelia by Sprengel (1817). Wallich (1819) described Didymocarpus Wall. and transferred all epithets under Henckelia to Didymocarpus. Later taxonomists followed this treatment for almost 180 years. Weber and Burtt (1998 [“1997”]) resurrected the former name Henckelia from synonymy and included about 180 species. In 2011, Weber et al. (2011) remodelled Henckelia to include 56 species formerly belonging to Henckelia sect. Henckelia, Chirita Buch.-Ham. sect. Chirita (excluding species

Received: 10.12.2019; Revised \& Accepted: 26.03.2020

Published Online: 11.05.2020 belonging to Damrongia Kerr ex Craib), the monotypic genus Hemiboeopsis W.T.Wang and excluded the species of Henckelia sect. Loxocarpus (R.Br.) A.Weber \& B.L.Burtt, sect. Didymanthus (C.B.Clarke) A.Weber \& B.L.Burtt, sect. Heteroboea (Benth.) A.Weber \& B.L.Burtt and sect. Glossadenia A.Weber \& B.L.Burtt. The genus under its current definition is distributed in Sri Lanka, India, Myanmar, Nepal, Bhutan, southern China, northern Vietnam, northern Laos, and northern Thailand. A few species were recently described bringing the total number to $c .70$ species worldwide (Manudev et al., 2012; Middleton et al., 2013; Kumar, 2014; Janeesha \& Nampy, 2015; Ranasinghe et al., 2016; Krishna \& Lakshminarasimhan, 2018; Borah et al., 2019; Cai et al., 2019; Sirimongkol et al., 2019; Yang et al., 2019; Kanthraj et al., this issue). It is the eighth largest genus in tribe Trichosporeae and tenth largest in subfamily Didymocarpoideae (Möller \& Clark, 2013). Thirty five species and one variety have so far been reported from India (Sinha \& Datta, 2016; Möller et al., 2017; Krishna \& Lakshminarasimhan, 2018; Borah et al., 2019; Kanthraj et al., this issue). Lectotypification of some species of this genus has been carried out by Vitek et al. (2000), Janeesha and Nampy (2016) and Ranasinghe et al. (2019). Henckelia is the largest genus of Gesneriaceae in India with a high proportion of endemism, particularly in South India. However, since Clarke's (1885[“1884”]) pioneering work in Hooker's Flora of British India, there has been no real revision of this genus in India. 
The purpose of the present study is to understand the diversity, distribution and taxonomy of the South Indian species of Henckelia, based on extensive field work and consultation of specimens and digital images deposited in major herbaria. Fifteen species are recognized in South India while two names are reduced to synonymy.

\section{Materials and Methods}

The materials for this study were obtained through field trips in various parts of South India. Data sheets were prepared for each population studied. Herbarium voucher specimens were prepared and deposited at Calicut University Herbarium (CALI). The specimens consulted, including types, are held at BLAT, BSD, BSI, CAL, CALI, DD, DEV, E, FRC, JCB, KFRI, MH, RHT, SKU, TBGT and online databases (https://plants.jstor.org; https:// www.ipni.org; https://www.tropicos.org; https:// www.biodiversitylibrary.org; https://www. wcsp.science.kew.org; https://www.samlinger. snm.ku.dk; https://www.linnean-online.org; http:// apps.kew.org/herbcat/navigator.do; https:// science.mnhn.fr; https://botanik.snm.ku.dk; https:// www.nhm.ac.uk). The acronyms of herbaria are as per Thiers (continuously updated). Detailed morphological descriptions were prepared after studying fresh and herbarium specimens using the terminology of Weber and Burtt (1998[“1997”]) and Simpson (2006). The genus description, preceding those of the species from South India, is applicable to the entire genus. Each species was identified by consulting types, and protologues. The nomenclature was updated with the latest literature. Colour photographs of the dissected floral parts were taken with a Leica EZ4HD stereo microscope (Mannheim, Germany). The distribution maps were prepared using the software QGIS 3.12.1-Bucuresti (QGIS Development Team, 2020) and conservation assessments were done using IUCN Standards and Petitions Subcommittee Criteria (2019). The information on phenology, ecology, habitat and distribution of each species was based on field studies and herbarium specimens consulted. The chromosome numbers and medicinal uses cited were gathered from the literature and referenced in the text.

\section{Taxonomic Treatment}

Henckelia Spreng., Anleit. Kenntn. Gew., ed. 2. 2(1): 402. 1817. Henckelia sect. Henckelia A.Weber \& B.L.Burtt, Beitr. Biol. Pflanzen 70: 334. 1998[“1997”]. Didymocarpus sect. Orthoboea Benth. in Bentham \& Hooker, Gen. Pl. 2(2): 1022. 1876. Type: Henckelia incana (Vahl) Spreng.

Chirita Buch.-Ham. ex D.Don, Edinburgh Philos. J. 7: 83. 1822. Chirita sect. Euchirita C.B.Clarke in A.DC. \& C.DC., Monogr. Phan. 5(1): 11. 1883, nom. illeg. Roettlera sect. Euchirita (C.B.Clarke) Fritsch in Engler \& Prantl, Nat. Pflanzenfam. IV/ 3b: 148. 1895. Didymocarpus sect. Euchirita (C.B.Clarke) Chun, Sunyatsenia 6: 294. 1946. Lectotype (designated by Burtt, 1954): Chirita urticifolia Buch.-Ham. ex D.Don (=Henckelia urticifolia (Buch.-Ham. ex D.Don.) A.Dietr.).

Perennial or annual herbs or subshrubs. Stem reduced (rosette-forming) or erect, ascending, rarely creeping. Leaves opposite, whorled or alternate by reduction of one leaf at a node in creeping species, sometimes one or two (rarely three) in a plant, short to long petiolate; laminae variable. Cymes axillary (or sometimes appearing terminal by reduction of the vegetative shoot); flowers (1)-10-38; bracts paired, ovate to linear, narrowly ovate or narrowly triangular, sometimes early caducous. Calyx of 5 sepals, free or fused into a tube for part of the length; lobes linear, lanceolate, ovate or triangular to narrowly triangular. Corolla zygomorphic, variously shaped, infundibuliform to campanulate, sometimes ventricose, 2-lipped, with upper lip of 2-lobes and lower lip of 3-lobes, colouration variable, often with yellow markings in throat. Stamens 2, usually included; filaments long, slightly bent (in South Indian species) to geniculate; anthers cohering, glabrous or pubescent; staminodes slightly bent, rarely straight or curved, with or without a prominent antherode, glabrous or pubescent. Disk cylindrical or cup shaped (absent 
in South Indian species). Ovary conical or ovoid, straight in relation to pedicel or with an angle between the pedicel and the ovary (usually between $120-135^{\circ}$ in South Indian Henckelia); stigma chiritoid or obliquely subcapitate (in South Indian species, except $H$. pradeepiana and $H$. repens). Capsules linear, rarely globose, stipitate or sessile, dehiscing loculicidally, splitting into 2 valves or opening only along the dorsal side (mostly in plagiocarpic ones), stipitate or sessile, orthocarpic or plagiocarpic (plagiocarpic in South Indian species). Seeds minute, numerous, ellipsoid.

Distribution: Sri Lanka, southern and northeastern India, Nepal, Myanmar, Bhutan, southern China, northern Vietnam, northern Laos and northern Thailand (Weber et al., 2011; Sirimongkol et al., 2019).

\section{Key to the species of Henckelia in South India}

1. Plants with creeping stems or tuberous herbs with 1-3 leaves ... 2

1. Rosette-forming herbs with many leaves in basal rosette ... 3

2. Tuberous herbs; leaves single (rarely up to 3); laminae broadly ovate or elliptic; capsules ovoid to sub-globose H. pradeepiana

2. Plants with creeping stems; leaves appearing alternate by reduction of one leaf in a pair at a node; laminae broadly ovate to sub-orbicular; capsules linear H. repens

3. Leaves distinctly petiolate; petioles not winged ... 4

3. Leaves sessile to ssub-sessile; if petioled, then winged ... 5

4. Peduncles scaly towards apex; filaments glabrous H. missionis

4. Peduncles not as above; filaments puberulent towards apex H. ovalifolia

5. Laminae obovate-spatulate or widely spatulate to elliptic
5. Laminae not as above, but of variable shapes 7

6. Peduncles 30-54 cm long, golden brown hairy H. macrostachya

6. Peduncles 10-18 cm long, glandular hairy H.wightii

7. Leaf margins doubly crenate or doubly serrate; ultimate segments irregular ... 8

7. Leaf margins crenate (occasionally doubly crenate in $H$. meeboldii); ultimate segments regular 11

8. Petioles lacerate; leaf bases lyrate; bracts pilose H. lyrata

8. Petioles not lacerate; leaf bases attenuate; bracts not as pilose 9

9. Leaves usually in whorls of three; calyx lobes hispid on both sides H. innominata

9. Leaves usually in whorls of four; calyx lobes tomentose outside, glabrous inside 10

10. Laminae ovate-widely ovate; bracts lanceolate H. fischeri

10. Laminae narrowly elliptic to widely elliptic; bracts ovate H. wayanadensis

11. Leaves usually in whorls of three 12

11. Leaves usually in whorls of two, decussate (occasionally whorls of three in $H$. humboldtiana) 13

12. Capsules $13-15 \mathrm{~mm}$ long, tomentose H. gambleana

12. Capsules 21-28 mm long, hispid H. incana

13. Bracts $10-21 \mathrm{~mm}$ long, elliptic to obovate; anthers bearded at the junction of the anther lobes and filament H. bracteata

13. Bracts 3-5.1 mm long, linear; anthers glabrous 14

14. Calyx lobes linear; corolla villous outside; ovary 8-9 mm long H. humboldtiana 
14. Calyx lobes ovate to lanceolate; corolla glandular hairy outside; ovary 4-5.5 mm long H. meeboldii

Henckelia bracteata Janeesha \& Nampy, Willdenowia 45(1): 53.2014. Type: INDIA, Kerala, Kottayam district, Kurisumala, near to $10^{\text {th }}$ Cross, $1100 \mathrm{~m}, 07.08 .2014$, A.P. Janeesha \& Santhosh Nampy 134270 (holo CALI!; iso CAL!, MH!) Fig. 1

Perennials, rosette-forming hairy herbs with rootstocks. Roots adventitious, thin. Leaves 8-14, basal, decussate; petioles 2-9 cm long, pubescent, winged; wings $2-3 \mathrm{~mm}$ broad; laminae ellipticovate, $5.5-12.5 \times 3-9 \mathrm{~cm}$, acute or obtuse to rounded at apex, narrowly cuneate to attenuate at base, tomentulose on both surfaces, but more so on the younger leaves and on lower veins, becoming less so with age, margins shallowly crenate, ultimate segments regular, surfaces usually rugose; veins usually 5 on each side, much branched and conspicuous beneath. Cymes axillary, dense, 1-11 per plant, 3-5(-6) times divided, 14-38flowered, pubescent; peduncles terete, $14-32 \mathrm{~cm}$ long, reddish brown, pubescent; bracts two at each branching, elliptic to obovate, 10-21 × 5-8 mm, obtuse to rounded at apex, margins crenate, tomentose; pedicels terete, $2-10 \mathrm{~mm}$ long, pubescent. Flowers 10-20 × 4-9 mm. Calyx 5- or rarely 6-partite, very deeply divided or basally connate; lobes ovate, unequal, posterior lobe smaller, $2.9-3.1 \times 1.2-1.6 \mathrm{~mm}$, others 3.3-4 × 1.6$2 \mathrm{~mm}$, slightly acute at apex, margins entire, reddish brown, white tomentose. Corolla zygomorphic, ventricose, two lipped, villous outside, glabrous inside; tube 7-9 × 5.5-7.5 mm, lilac to pale lilac with a yellow patch in the throat; lobes ovate to rounded at apex, lilac to pale lilac, upper lip of two lobes, 5.2-5.9 $\times 7.6-8.1 \mathrm{~mm}$, lower lip of three lobes, 5.6-7 × 7.4-8.3 mm. Stamens 2, adnate to the base of corolla, included; filaments $4-6 \mathrm{~mm}$ long, glabrous or sparsely pubescent towards the region of the connective, green on the top and colourless towards base; anthers reniform, 1.5-1.8 $\times 0.8-0.9 \mathrm{~mm}$, milky white, bearded at the junction of the anther lobes and filament. Staminodes 3 (sometimes 2); filaments $2.5-3 \mathrm{~mm}$ long, hyaline, glabrous; antherodes not seen. Ovary ovoid to oblong, 5-7 ×1.25-1.5 mm, green, pubescent; style terete, $2-2.5 \mathrm{~mm}$ long, green, glabrous; stigma obliquely subcapitate, $0.8-1.1 \times 0.5-0.82 \mathrm{~mm}$, papillate, greenish white. Capsules cylindrical, straight or randomly curved, $18-30 \times 1.6-2 \mathrm{~mm}$, plagiocarpic, dehiscing loculicidally along the dorsal side, reddish brown, tomentulose with persistent stigma. Seeds numerous, elliptic, 0.4-0.6 $\times 0.2-0.25 \mathrm{~mm}$, slightly acute at apex, truncate at base; testa dark brown to black, reticulate.

Flowering \& fruiting: Flowering fom July to September and fruiting from August to October.

Habitat: In wet humus in granite rock crevices, at altitudes $1100 \mathrm{~m}$ above sea level, along with Mitracarpus hirtus DC. (Rubiaceae), Porella sp. (Porellaceae) and species of moss, Selaginella sp. (Selaginellaceae) and grasses (Poaceae).

Distribution: Endemic to the southern Western Ghats, hitherto known only from the type locality and adjoining areas (Fig. 6).

Specimens examined: INDIA, Kerala, Kottayam district, Kurisumala, 17.08.2013, K.M. Manudev \& A.P. Janeesha 134206, 134209; on the way to $6^{\text {th }}$ cross, 07.08.2014, A.P. Janeesha \& A.P. Roshan 134268; near to $6^{\text {th }}$ cross, 07.08.2014, A.P. Janeesha \& A.P. Roshan 134269; near $7^{\text {th }}$ cross, 26.06.2016, P.S. Sreeja \& A.P. Janeesha 137659; near $10^{\text {th }}$ cross, 26.06.2016, P.S. Sreeja \& A.P. Janeesha 137660; on the way to Elaveezhapoonchira, 28.06.2016, A.P. Janeesha \& Santhosh Nampy 137665 (CALI).

Conservation status: The species can be found at two localities in Kurisumala and Elaveezhapoonchira about $20 \mathrm{~km}$ apart, with an estimated Extent of Occurrence (EOO) of $34 \mathrm{~km}^{2}$. Eighty two mature plants were observed across these two localities and the plants at Kurisumala and the series of crosses represent a continuous population. A continuing decline in population size has been observed over three years because the localities are tourist destinations. Therefore, this species is assessed here as Endangered (EN), B1ab(ii,iii,iv,v), B2ab 


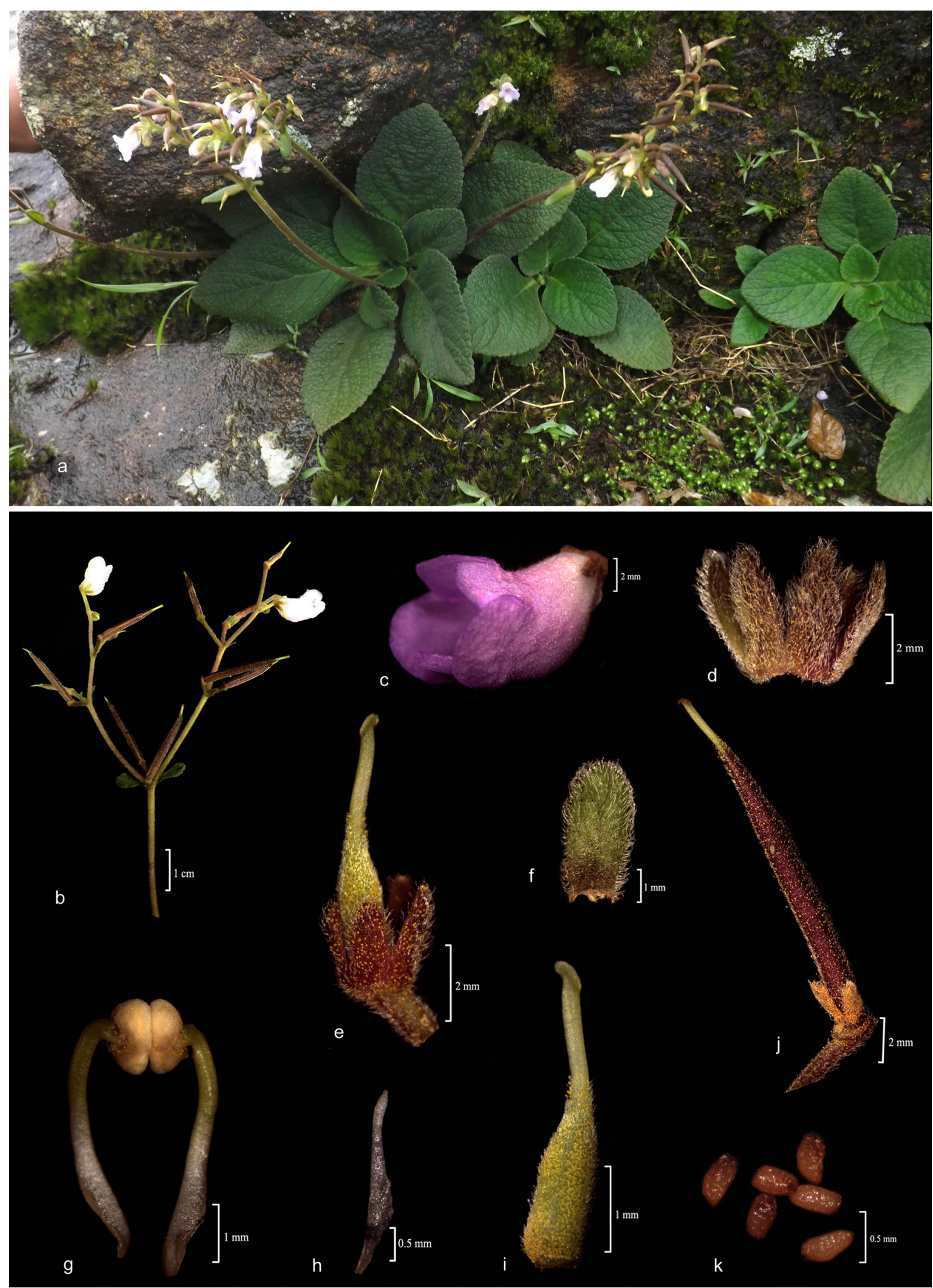

Fig. 1. Henckelia bracteata Janeesha \& Nampy: a. Habit and habitat; b. A portion of the inflorescence; c. Flower; d. Calyx; e. Calyx with pistil; f. Bract; g. Stamens; h. Staminode; i. Pistil; j. Capsule; k. Seeds (from A.P. Janeesha \& Santhosh Nampy 134270). 
(ii,iii,iv,v), D, according to IUCN's guidelines (IUCN, 2019).

Notes: This species is morphologically close to $H$. humboldtiana in the shape of leaves, presence of bracts and much longer scapes than the leaves (Janeesha \& Nampy, 2015; Janeesha, 2017). However, it is distinguishable by its thick laminae with narrowly cuneate to attenuate base, larger, elliptic to obovate bracts, ovate calyx lobes that do not reach the full length of the ovary and hairs at the junction of the anther lobes and filaments. Henckelia bracteata plants are robust and dull green throughout while the peduncles, pedicels, calyx and capsules are usually reddish brown. Although the margins of the laminae are crenate, the crenation is shallow when compared to H. humboldtiana. When grown in the greenhouse at low altitudes, the colour of the corolla changes from pale lilac to dark lilac to violet.

Henckelia fischeri (Gamble) A.Weber \& B.L.Burtt, Beitr. Biol. Pflanzen 70: 344. 1998[“1997"]; T.S.Nayar et al., Fl. Pl. Western Ghats 1: 527. 2014. Didymocarpus fischeri Gamble, Bull. Misc. Inform. Kew 1923(93): 117. 1923, Fl. Madras 2: 988. 1924; A.N.Henry et al., Fl. Tamil Nadu Ind., Ser I: Analysis 2: 132. 1987; M.Ahmedullah \& M.P.Nayar, Endemic Pl. Indian Region 1: 142. 1987; M.Mohanan \& A.N.Henry, Fl. Thiruvananthapuram 337. 1994; Sasidh. \& Sivar., Fl. Pl. Thrissur Forest 329. 1996; Sasidh., Biodivers. Doc. Kerala 6: 331. 2004; T.S.Nayar et al., Fl. Pl. Kerala 342. 2006. Lectotype (first-step designated by Vitek et al., 2000; second-step designated here): INDIA, Tamil Nadu, Coimbatore district, Anamalai hills, 500-600 m, 02.08.1915, C.E.C. Fischer s.n. (K [K000450837 digital image!]; isolecto K [K00122291 digital image!]).

Figs. $2 \& 3$

Perennials, rosette-forming hairy herbs with rootstocks. Roots adventitious, thin. Leaves 12-20, basal, in whorls of four; petioles $0.7-5 \mathrm{~cm}$ long (sometimes up to $7.5 \mathrm{~cm}$ ), pubescent, winged; wings 4-12 mm broad; laminae ovate-widely ovate, $8-23 \times 6-12 \mathrm{~cm}$, obtuse to slightly acute at apex, attenuate at base, tomentose on both surfaces, with small white patches on the upper surfaces, golden brown on younger leaves and on lower veins, becoming less so with age, margins doubly crenate, ultimate segments irregular, surfaces usually rugose; veins usually 6-8 on each side, much branched and conspicuous beneath. Cymes axillary, 3-7 per plant, 4-7 times divided, 16-37-flowered; peduncles terete, 17-38 cm long, green-pale reddish brown, glandular hairy; bracts two at each branching point, lanceolate, 2-4 × c. $1 \mathrm{~mm}$, acute at apex, margins entire, tomentose, with a tuft of hairs at the apex, green to purple; pedicels terete, 6-24 mm long, glandular hairy. Flowers 11-17 × 9-22 mm. Calyx 5 -partite, very deeply divided or basally connate; lobes linear to broadly lanceolate-ovate, unequal, posterior lobe smaller, 2.5-3.8 × 0.8-1.6, others 3.6$6 \times 1.2-1.9 \mathrm{~mm}$, slightly acute or obtuse to rounded at apex, margins entire, reddish brown hairy. Corolla zygomorphic, ventricose, two lipped, glandular hairy outside, glabrous inside; tube 6.8$12 \times 5.7-7.9 \mathrm{~mm}$, dark to pale lilac with a yellow blotch at the throat; lobes rounded or very widely ovate with undulate margins, dark to pale lilac, upper lip of two lobes, 6.6-7.3 × 7.7-10 mm, lower lip of three lobes, $6.9-7.8 \times 8.6-10.9 \mathrm{~mm}$. Stamens 2 , adnate to the base of corolla, included; filaments 4-5.3 mm long, glabrous, yellow; anthers reniform, 1.5-2.75 × 0.75-1.16 mm, milky white to cream. Staminodes 2 (the lateral ones), sometimes 3; filaments 2.4-4 $\mathrm{mm}$ long, hyaline, glabrous; antherodes sometimes present. Ovary ovoid to oblong, 3.7-6 × 1-1.27 mm, green, glandular hairy; style terete, 1-4.5 mm long, green, glabrous towards the apex, basally glandular hairy; stigma obliquely subcapitate, $0.65-0.87 \times 0.9-1.1 \mathrm{~mm}$, slightly papillate, pale green. Capsules cylindrical, straight or randomly curved, 14-37 × 1.8-2.4 mm, plagiocarpic, dehiscing loculicidally along the dorsal side, reddish brown, glandular hairy with persistent stigma and calyx. Seeds numerous, elliptic, $0.57-0.69 \times 0.22-0.29 \mathrm{~mm}$, slightly acute at apex, truncate at base; testa dark brown, reticulate. Flowering \& fruiting: Flowering from May to October and fruiting from July to December. 


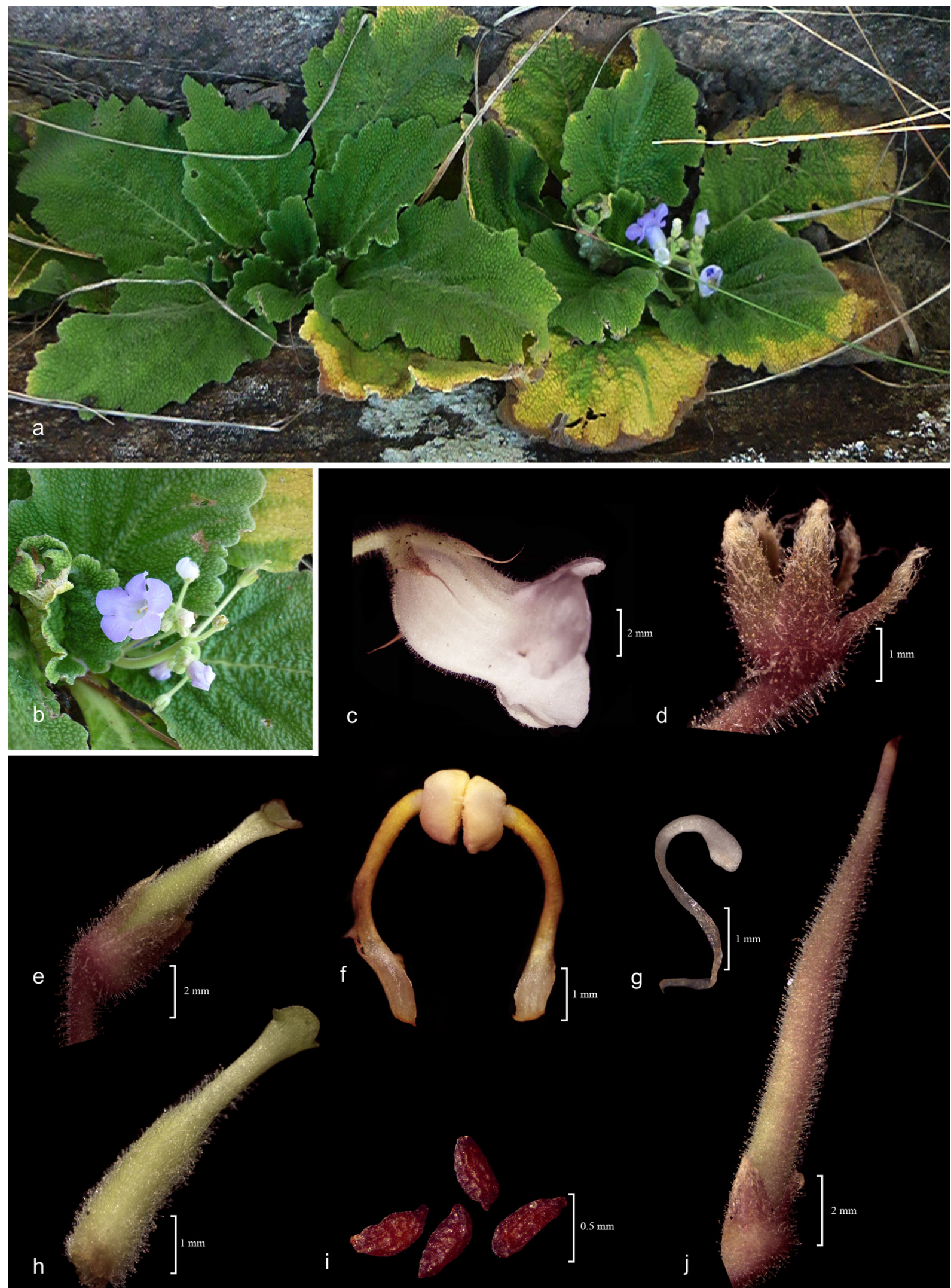

Fig. 2. Henckelia fischeri (Gamble) A.Weber \& B.L.Burtt: a. Habit and habitat; b. Inflorescence; c. Flower; d. Calyx; e. Calyx with pistil; f. Stamens; g. Staminode; h. Pistil; i. Seeds; j. Immature capsule (a \& b from A.P. Janeesha, Habeeb Rahman \& A. Kabeer 134216; c-j from A.P. Janeesha \& Santhosh Nampy 134224). 


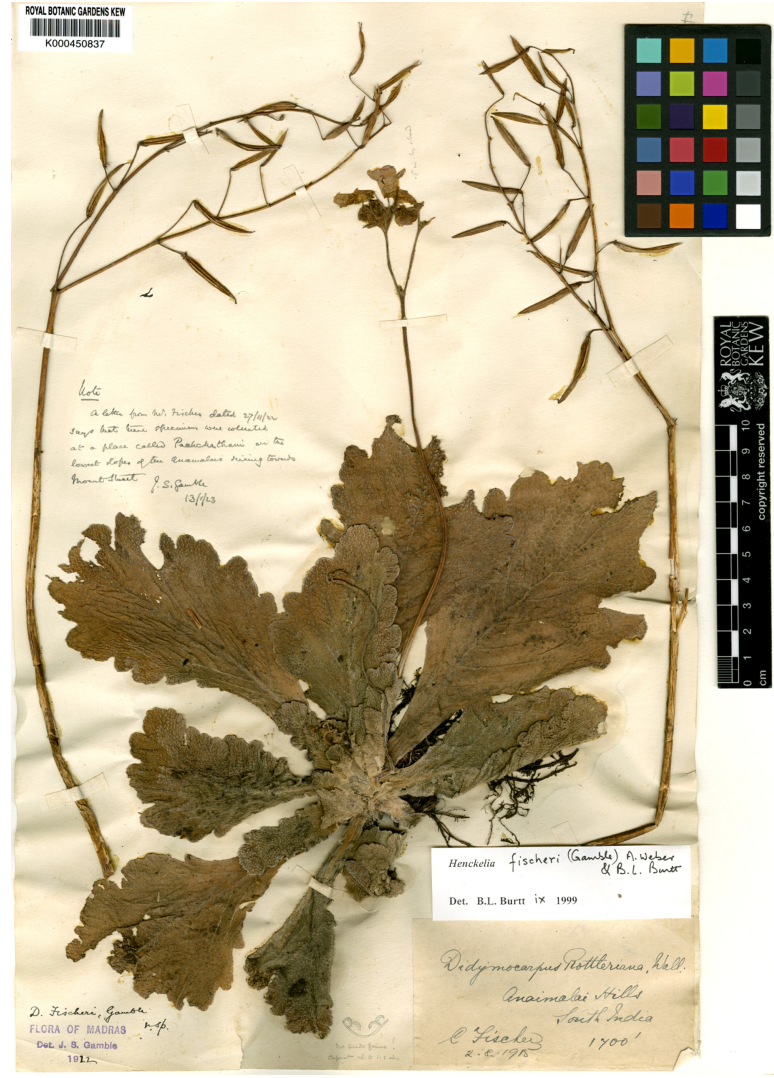

Fig. 3. Lectotype of Henckelia fischeri (Gamble) A.Weber \& B.L.Burtt. (C) The Board of Trustees for The Royal Botanic Gardens, Kew. Reproduced with permission.

Habitat: On moist slopes and in rock crevices, usually at an altitude above $850 \mathrm{~m}$, in association with Adiantum philippense L., A. concinnum Willd., Hemionitis arifolia (Burm.) T.Moore (all Pteridaceae), Spermacoce exilis (L.O.Williams) C.D.Adams, Mitracarpus hirtus DC., Oldenlandia umbellata L. (all Rubiaceae), Leucas aspera Link. (Lamiaceae) and a few grass species.

Distribution: Endemic to southern Western Ghats (Fig. 6).

Specimens examined: INDIA, Kerala, Idukki district, Kottamala, 16.05.1995, Jomy Augustine 15309 (CALI); near Idukki dam, 24.08.1981, V.S. Raju 71173 (MH); Santhanpara, 2016, P. Soumya \& A.P. Janeesha 137678 (CALI); Palakkad district, Karimala-grassland, 26.09.2013, A.P. Janeesha \& Santhosh Nampy 134227 (CALI); Nelliyampathy, 29.06.1994, A.E. Shanavas Khan \& E.S. Santhosh Kumar 20100 (TBGT); Ibid., s.d., V.P.K. Nambiar
1067 (KFRI digital image); Parambikulam-way to Thunakkadavu dam view point, 24.09.2013, A.P. Janeesha \& Santhosh Nampy 134222; Seetharkundnear the water fall, 17.09.2013, A.P. Janeesha, Habeeb Rahman \& A. Kabeer 134216; Vengoli hill top, 25.09.2013, A.P. Janeesha \& Santhosh Nampy 134224 (CALI); Pathanamthitta district, Achankovil, s.d., A.G. Pandurangan 14432 (TBGT). Tamil Nadu, Coimbatore district, Anamalai, 1857, s.coll. s.n. (MH); Nilgiri district, Kodanad, 12.11.1970, E. Vajravelu 36854 (MH); Tenkasi district, Courtallam, s.d., K. Narayana Iyer 01874 (TBGT); Theni district, Meghamalai, 25.08.1985, K. Ravikumar 2380 (MH); Tirunelveli district, Kularatti estate, 21.12.1980, E. Vajravelu $76416(\mathrm{MH})$.

Conservation status: The species is widely distributed in Kerala with a few localities in Tamil Nadu with around twelve localities. The Extent of Occurrence (EOO) is estimated to be $c .5600 \mathrm{~km}^{2}$. The Area of Occupancy (AOO) is likely less than $50 \mathrm{~km}^{2}$, since the plants occur in localized populations. For instance, in Kerala five locations were surveyed and a total of only 136 mature plants were found. The locations in Kerala and Tamil Nadu are important tourist destinations. Therefore, there is the potential of disturbance due to tourist activities with detrimental outcomes for the number of plants and localities. Even though the species is widely distributed, because of the likely small AOO the species could be categorized at present as Endangered (EN), B2ab(ii,iii,v), according to IUCN (2019) criteria.

Notes: This is a fairly large plant with deeply crenate leaves and many-flowered, long scapes. The flowers are deeply pouched and horizontally oriented. Their scapes are pale greenish-red under natural conditions, but bright green when grown in the greenhouse.

Typification: This species was described by Gamble (1924) based on specimens collected by C.E.C. Fischer from Anamalai hills. Vitek et al. (2000) cited "South India, Anamalai hills, Coimbatore district, 500-600 m alt., 02.08.1915, C.E.C. Fischer (K)” as the type. While searching for the type specimens, 
two relevant sheets were found in K (K000450837, K00122291). Both sheets were annotated, "Didymocarpus Rottlerianus, Wall., Anamalai hills, South India, 1700 ft., C. Fischer, 2.8.1915”. The sheet K000450837 has a flowering plant, and two fruiting scapes separately mounted on it, but contains a note by Gamble "a letter from Mr. Fischer dated 27.11.22 says that three specimens were collected at a place called Pachchathani on the lowest slopes of the anamalais ...... towards mount Stuart, 13.1.23" while the other sheet has two flowering plants. According to Art. 9.17 of ICN (Turland et al., 2018), we take a second step lectotypification by narrowing them to a single specimen. We chose K000450837 that agrees with the protologue and has the inscription by Gamble, while the other specimen (K00122291) is designated as isolectotype.

Henckelia gambleana (C.E.C.Fisch.) A.Weber \& B.L.Burtt, Beitr. Biol. Pflanzen 70: 345. 1998[“1997”]; T.S.Nayar et al., Fl. Pl. Western Ghats 1: 528. 2014. Didymocarpus gambleanus C.E.C.Fisch., Bull. Misc. Inform. Kew 1938: 36. 1938 (as 'gambleana'); A.N.Henry et al., Fl. Tamil Nadu Ind., Ser I: Analysis 2: 132. 1987; M.Ahmedullah \& M.P.Nayar, Endemic Pl. Indian Region 1: 143. 1987. Didymocarpus rottlerianus Wall. ex R.Br. var. lanuginosus C.B.Clarke in A.DC. \& C.DC., Monogr. Phan. 5 (1): 102. 1883. Didymocarpus tomentosus Wight var. lanuginosus (C.B.Clarke) C.B.Clarke in Hook.f., Fl. Brit. India 4: 353. (1885[“1884”]). Didymocarpus lanuginosus (C.B.Clarke) Wight ex Gamble, Fl. Madras 988. 1924, nom illeg., non Wall. ex R.Br. 1839, non Wall. ex A.DC. 1845. Lectotype (first-step designated by Vitek et al., 2000; second-step designated here): s.loc., s.d., Wight 561 (K [K000858182 digital image!]; isolecto E [E00627530!], K [K000858183 digital image!]).

Figs. 4 \& 5

Perennials, rosette-forming hairy herbs with rootstocks. Roots adventitious, thin. Leaves 6-12, basal, usually in whorl of three; petioles $0.7-1.8 \mathrm{~cm}$ long, younger leaves subsessile, pubescent, winged; wings 5-8 $\mathrm{mm}$ broad; laminae widely ovate, 4-7.7 $\times 3.2-6 \mathrm{~cm}$, acute to obtuse at apex, narrowly cuneate to attenuate at base, hispid on the upper surface, golden brown hairy on lower surface, but more so on younger leaves and lower veins, becoming fewer with age, margins crenate, ultimate segments regular, surfaces usually rugose; veins usually 4 on each side, much branched and conspicuous beneath. Cymes axillary, 2-4 per plant (rarely up to 11), 3-5 times divided, 12-36flowered; peduncles terete, 8-19 cm long, green to reddish brown, golden brown hairy; bracts two at each branching point, lanceolate to triangular, 3-3.5 $\times 0.36-0.4 \mathrm{~mm}$, obtuse to slightly acute at apex, margins entire, white tomentose; pedicels terete, 4-14 mm long, pubescent. Flowers 8-11 $\times$ 5-6.5 mm. Calyx 5-partite, very deeply divided or basally connate; lobes lanceolate to narrowly oblong, unequal, posterior lobe smaller, 2.7-3 $\times$ $0.42-0.53 \mathrm{~mm}$, others $2.9-3.2 \times 0.5-0.68 \mathrm{~mm}$, obtuse at apex, margins entire, reddish brown, white hairy. Corolla zygomorphic, ventricose, two lipped, villous outside, glabrous inside; tube 7-8 $\times$ 5.8-6.4 mm, lilac to pale lilac with a yellow patch in the throat; lobes widely ovate, rounded to truncate at apex, lilac to pale lilac, upper lip of two lobes, $3.8-4.3 \times 3.5-4.1 \mathrm{~mm}$, lower lip of three lobes, 4.2-5.1 $\times 4-5 \mathrm{~mm}$. Stamens 2 , adnate to the base of corolla, included; filaments $2.2-2.7 \mathrm{~mm}$ long, glabrous, green on the top and colourless towards base; anthers reniform, 1.3-1.5 × 0.6-0.7 $\mathrm{mm}$, milky white, glabrous. Staminodes 2 ; filaments 1.6-1.8 mm long, hyaline, glabrous; antherodes not seen. Ovary narrowly ovoid to oblong, 4-4.7 ×0.9$1 \mathrm{~mm}$, green, pubescent; style terete, $2.6-2.9 \mathrm{~mm}$ long, green, glabrous; stigma obliquely subcapitate, $0.21 \times 0.5 \mathrm{~mm}$, papillate, pale green. Capsules cylindrical, straight, 13-15 mm long, plagiocarpic, dehiscing loculicidally along the dorsal side, reddish brown, tomentose with persistent stigma and calyx. Seeds numerous, elliptic, reticulate.

Flowering \& fruiting: Flowering from July to late September and fruiting from September to November (sometimes up to March). 

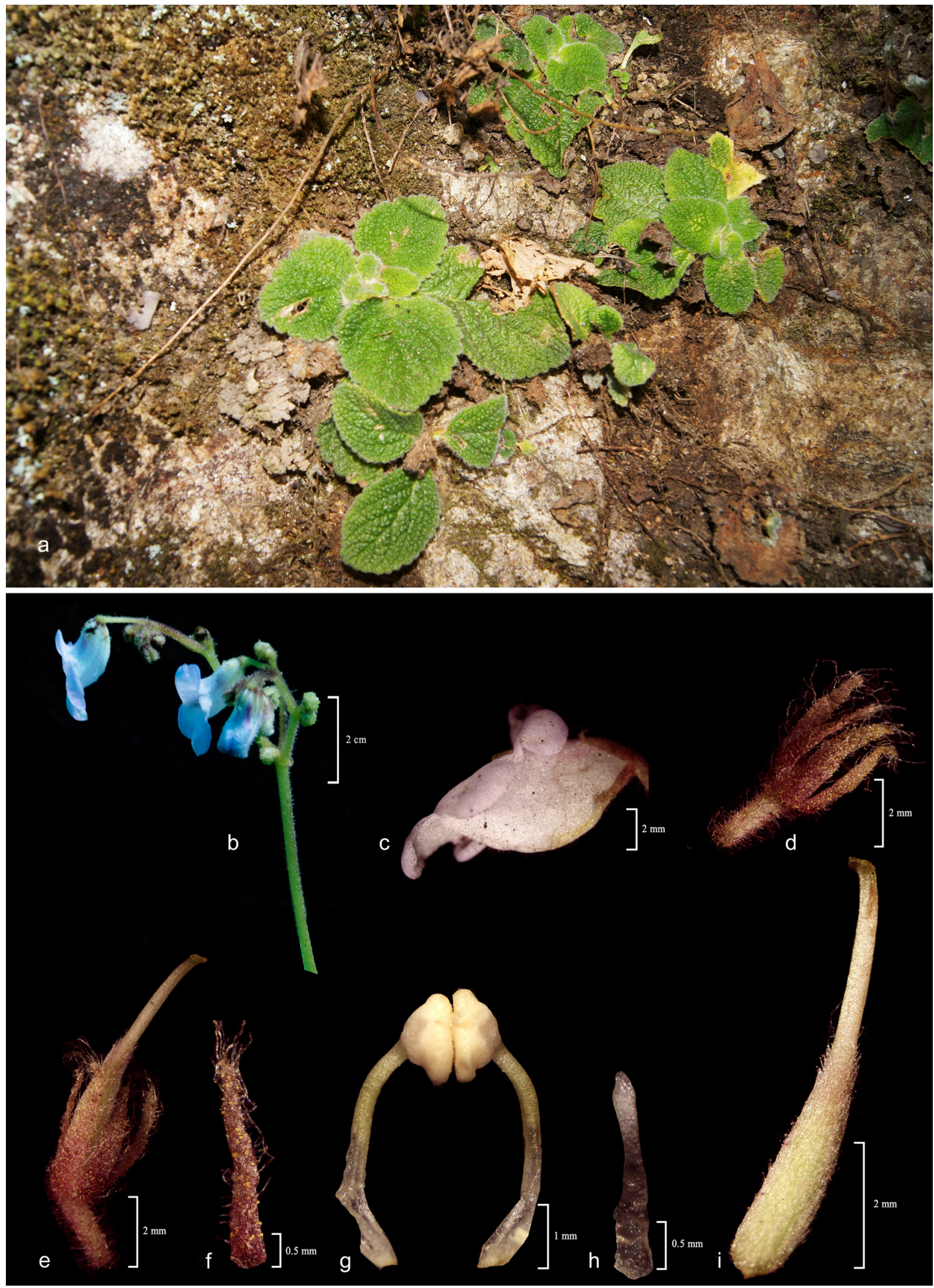

Fig. 4. Henckelia gambleana (C.E.C.Fisch.) A.Weber \& B.L.Burtt: a. Habit and habitat; b. A portion of the inflorescence; c. Flower; d. Calyx; e. Calyx with pistil; f. Bract; g. Stamens; h. Staminode; i. Pistil (from A.P. Janeesha \& Santhosh Nampy 137677). 


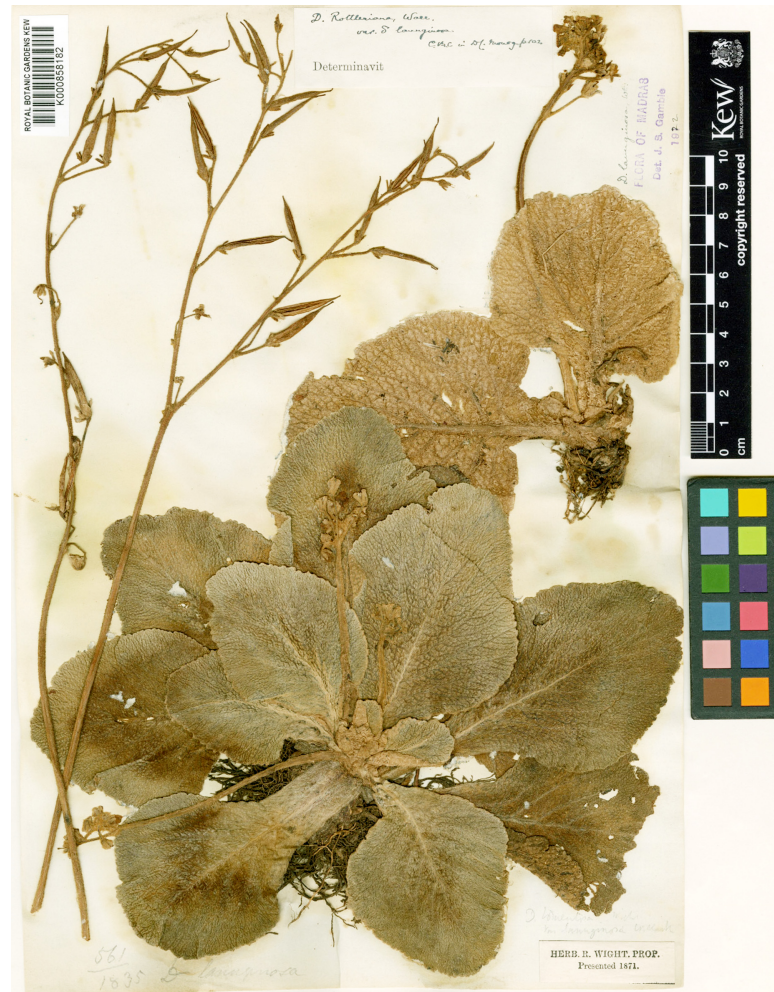

Fig. 5. Lectotype of Henckelia gambleana (C.E.C.Fisch.) A.Weber \& B.L.Burtt. (C) The Board of Trustees for The Royal Botanic Gardens, Kew. Reproduced with permission.

Habitat: On moist hill slopes, along with Adiantum raddianum C.Presl (Pteridaceae) and a few species of moss.

Distribution: Endemic to the southern Western Ghats (Fig. 6).

Specimens examined: INDIA, Tamil Nadu, Coimbatore district, Bolampatti valley, 24.02.1917, C.E.C. Fischer 4059 (FRC); Kanyakumari district, Muthukuzhivayal grass land, 15.03.1979, A.N. Henry 60703 (MH); Tenkasi district, CourtallamThekkumalai, 11.11.1984, Jolly Jacob 4072; Ibid., 11.11.1984, A.R. Sheela 3677; Ibid., 11.11.1984, K. Leela 4344; Ibid., 11.11.1984, A.M. Rema 4878 (CALI); Tirunelveli district, Agasthyamalai peak, 01.07.1964, A.N. Henry \& M. Chandrabose 19215; Ibid., 24.08.1963, A.N. Henry 17317; Kannikathi, 13.06.1899, C.A. Barber 506; Neelikkattu, 11.10.1992, R. Gopalan 99487 (MH); on the way to Kakkachi-near Manjolai estate board, 21.07.2016, A.P. Janeesha \& Santhosh Nampy 137677 (CALI); Sevgaltai, 24.09.1915, s.coll. 12477 (MH);
Upper Kodayar, 02.09.1981, K.M. Matthew 17831 (RHT); way to Kannikkatti, 05.07.1964, A.N.Henry \& M. Chandrabose $19292(\mathrm{MH})$. s.loc., s.d., T. Surendran 5105 (CALI).

Conservation status: The species occurs in several populations in three to four localities widely disjunct in the SW and NW of Tamil Nadu. Because of this disjunction, the Extent of Occurrence (EOO) is with of c. $3500 \mathrm{~km}^{2}$ large. The authors have observed only 27 mature plants in one population in Kakkachi, in the Tirunelveli district in Tamil Nadu. This might suggests that the populations in general are rather small. However, since the status of other localities is unknown and potentially intervening localities may exist, the species is best categorised as Data deficient (DD).

Notes: This species can be easily differentiated from the morphologically allied $H$. incana by its broadly winged petioles, and much shorter (13-15 mm long), tomentose capsules.

Typification: Henckelia gambleana was originally described as a variety of Didymocarpus rottlerianus Wall. ex R.Br. by Clarke (1883) based on Wight's manuscript name D. lanuginosus. Clarke (1885[“1884”]), subsequently treated it as a variety of D. tomentosus Wight (as D. tomentosus var. lanuginosus). Gamble (1924), however, elevated it to a distinct species (as D. lanuginosus). Gamble's D. lanuginosus, being a later homonym of $D$. lanuginosus Wallich ex Candolle (1845), was renamed as D. gambleanus $(=H$. gambleana) by Fischer (1938). While searching for the type specimens, three sheets were found, two in K (K000858182, K000858183) and one in E (E00627530). The sheet K000858182 with HRWP label is annotated "D. lanuginosa, 561/1835" by Wight. This sheet has two plants and two scapes separately mounted on it. The other sheet K000858183 has a field label, Courtallam 561. There is one plant in vegetative stage and a scape separately mounted on it. The specimen in $\mathrm{E}$ (E00627530) has three plants, two in vegetative stage and another flowering plant and a separate 


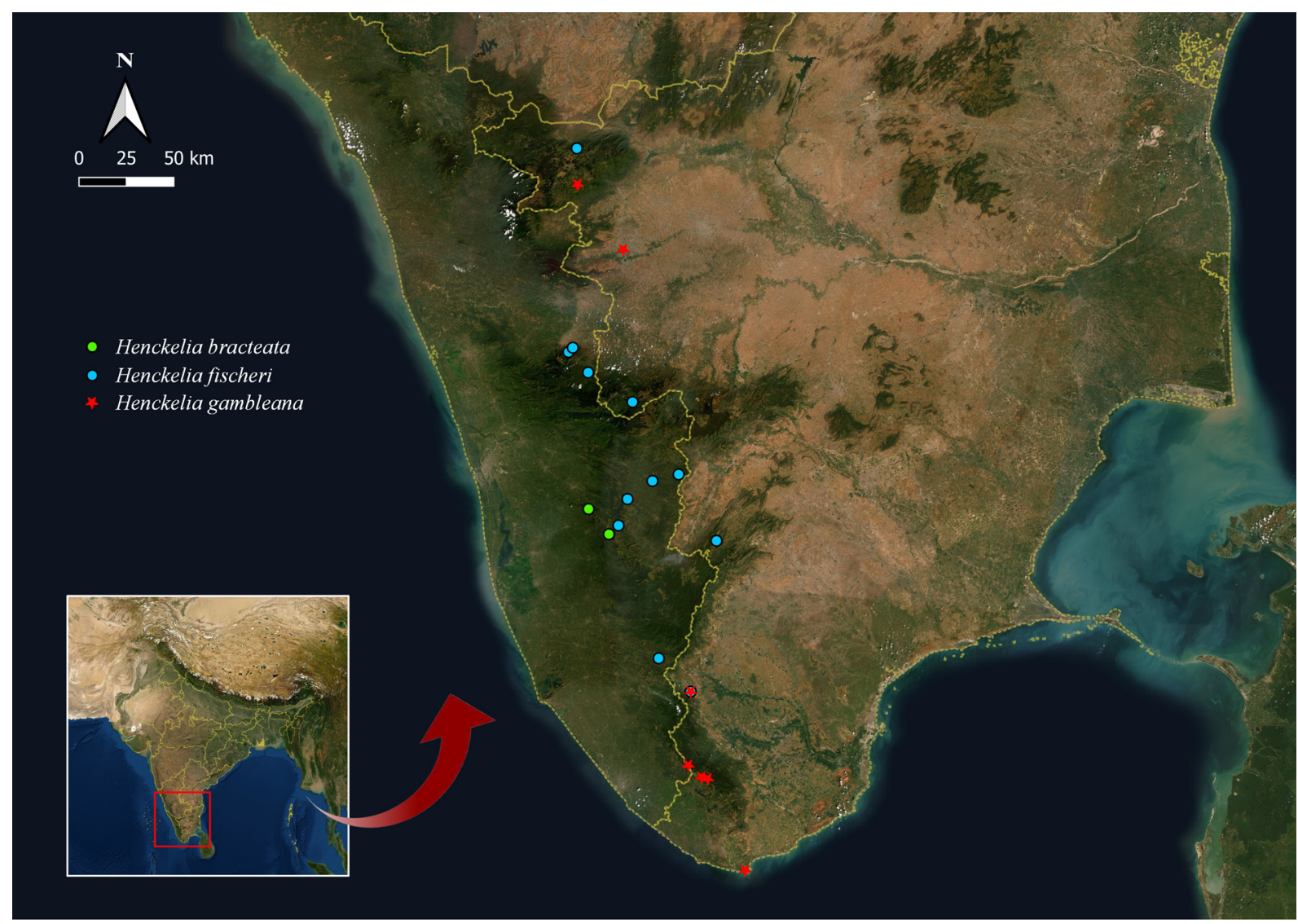

Fig. 6. Distribution of Henckelia bracteata Janeesha \& Nampy, H. fischeri (Gamble) A.Weber \& B.L.Burtt and H. gambleana (C.E.C.Fisch.) A.Weber \& B.L.Burtt in South India (drawn using QGIS v.3.12.1).

scape. This sheet also has a field label 561, Courtallam July 1835. Vitek et al. (2000) cited "Wight $561(\mathrm{~K})$ " as the type. According to Art.9.17 of ICN (Turland et al., 2018), we take a secondstep lectotypification by narrowing them to a single specimen at K (K000858182) that agrees with the protologue and designate the rest of the specimens as isolectotypes.

Henckelia humboldtiana (Gardner) A.Weber \& B.L.Burtt, Beitr. Biol. P flanzen 70: 346. 1998 [“1997”]; T.S.Nayar et al., Fl. Pl. Western Ghats 1: 528. 2014. Didymocarpus humboldtianus Gardner, Calcutta J. Nat. Hist. 6: 477. 1846; C.B.Clarke in Hook.f., Fl. Brit. India 4: 353. 1885[“1884”]; Gamble, Fl. Madras 988. 1924; W.L.Theob. \& Grupe in Dassan. \& Fosberg, Rev. Handb. Fl. Ceylon 3: 84. 1981; A.N.Henry et al., Fl. Tamil Nadu Ind., Ser I: Analysis 2: 132. 1987; Manil., Fl. Silent Valley 200. 1988; Sasidh., Biodivers. Doc. Kerala Part 6: Fl. Pl. 332. 2004; N.Anilkumar et al.,
Fl. Pathanamthitta Dist. 370. 2005; T.S.Nayar et al., Fl. Pl. Kerala 342. 2006. Roettlera humboldtiana (Gardner) Kuntze, Revis. Gen. Pl. 2: 476. 1891. Lectotype (designated by Janeesha \& Nampy, 2015): SRI LANKA (CEYLON), Rambodde, Gardner 600 (K [K000858189 digital image!]; isolecto K [K000858188 digital image!]).

Didymocarpus primulifolius Gardner, Calcutta J. Nat. Hist. 6: 478. 1846, nom. illeg. Lectotype (designated by Ranasinghe et al., 2019): SRI LANKA, Hantane range, Near Candy, s.d., Gardner 601 (K [K000858191 digital image!]; isolecto BM [BM000617482 digital image!], K [K000858192 digital image!]).

Fig. 7

Perennial, rosette-forming hairy herbs with rootstocks. Roots adventitious, thin. Leaves 4-12, basal, decussate, rarely in whorls of three; petioles 2-6 cm long, longer in older leaves, narrowly winged, pubescent; wings $0.5-2 \mathrm{~mm}$ broad; 


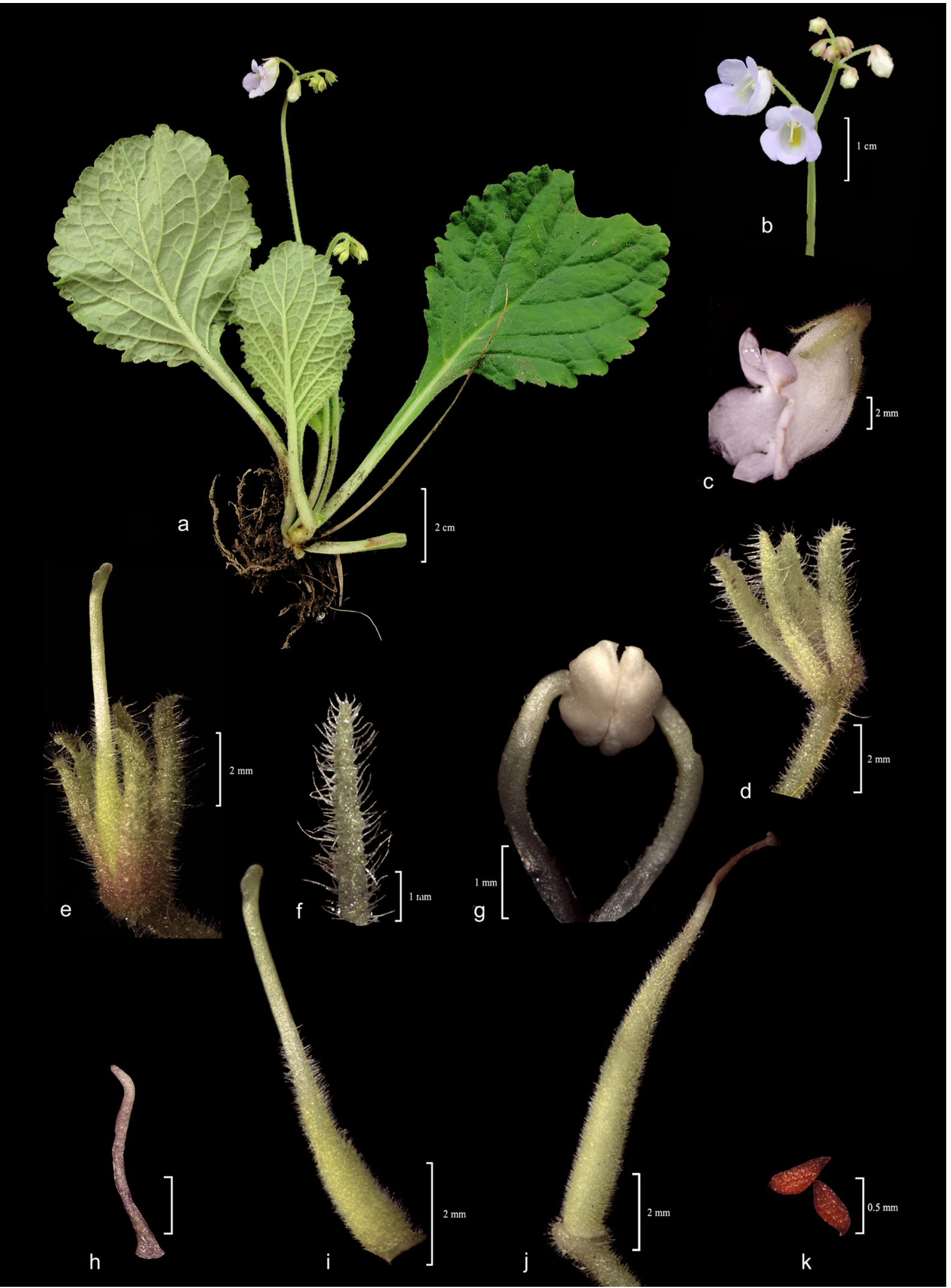

Fig. 7. Henckelia humboldtiana (Gardner) A.Weber \& B.L.Burtt: a. Habit; b. A portion of the inflorescence; c. Flower; d. Calyx; e. Calyx with pistil; f. Bract; g. Stamens; h. Staminode; i. Pistil; j. Immature capsule; k. Seeds (a from A.P. Janeesha \& A.J. Robi 134201; b-k from A.P. Janeesha, Habeeb Rahman \& S. Syam Radh 134221). 
laminae elliptic-ovate, $5-8 \times 2.1-5.9 \mathrm{~cm}$, slightly acute at apex, attenuate at base, margins crenate, ultimate segments regular, slightly undulate, surfaces rugose, tomentulose on both surfaces, but more so on the younger leaves and on lower veins, becoming less so with age; veins 5-6 pairs on each side, much branched and conspicuous beneath. Cymes axillary, lax, 1-4 per plant, 2-3 times divided, 6-10-flowered, pubescent; peduncles terete, 8-12 cm long, pale green, pubescent; bracts two at each branching point, linear to oblong, 4.2$5.1 \mathrm{~mm}$ long, obtuse at apex, tomentose; pedicels terete, 5-10 mm long, slender, pubescent. Flowers 10-12 × 6-7.5 mm. Calyx 5-partite, free or very deeply divided; lobes linear, unequal, posterior lobe smaller, 4.3-4.8 × 0.6-0.75 mm, others 5.1-6 $\times$ $0.72-0.9 \mathrm{~mm}$, slightly acute at apex, margins entire, pale green with a pinch of reddish brown at the base, tomentose. Corolla, zygomorphic, ventricose, two lipped, villous outside, glabrous inside; tube 5-9 × 4.2-5.5 mm, pale lilac to white with a yellow patch in the throat; lobes rounded, rounded at apex, white to pale lilac, upper lip of two lobes, 3.1-3.4 $\times$ 3.2-3.7 mm, lower lip of three lobes, 3.3-4.4 × 3.5$5.6 \mathrm{~mm}$. Stamens 2, adnate to the base of corolla, included; filaments $5 \mathrm{~mm}$ long, slightly bearded, pale green at the top, base hyaline; anthers reniform, c. $1.3 \times 0.8 \mathrm{~mm}$, milky white, glabrous. Staminodes 2. Ovary ovoid to oblong, 8-9 × 1-1.1 mm, green, pubescent; style terete, 3.5-4 mm long, green, base slightly hairy; stigma obliquely subcapitate, 0.65$0.8 \times 0.34-0.42 \mathrm{~mm}$, papillate, pale green. Capsules linear, straight or slightly curved, 12-21 × 1.1-1.6 $\mathrm{mm}$, plagiocarpic, dehiscing loculicidally along the dorsal side, pale green, tomentulose with persistent stigma. Seeds numerous, elliptic, 0.32-0.5 × $0.1-$ $0.19 \mathrm{~mm}$, slightly acute at apex, truncate at base; testa dark brown to black, reticulate.

Flowering \& fruiting: Flowering from May to September and fruiting from July to November.

Habitat: On moist slopes or on rocks, at medium elevations of $350 \mathrm{~m}$, along with Selaginella sp. (Selaginellaceae), Pteris sp. (Pteridaceae), Impatiens gardneriana Wight, I. acaulis Arn. (both Balsaminaceae) and a few grass species.
Distribution: South India and Sri Lanka (Fig. 11).

Specimens examined: INDIA, Kerala, Ernakulam district, Neriyamangalam, 20.08.1965, K.M. Sebastine 25087 (MH); Idukki district, Munnar, 08.11.1985, P.C. Binoy 05047 (TBGT); Kollam district, Thenmala, 01.10.1960, Joseph 2, 3 (BSI, E); Kottayam district, Pambanar, 24.05.1964, K. Vivekananthan 23998; Pullupara, 24.09.1964, K. Vivekananthan 21314 (MH); Vagamon, 03.10.2015, C. Pramod \& A.P. Janeesha 137644, 137647 (CALI); Kozhikode district, Kakkayam, 20.07.2013, A.P. Janeesha \& A.J. Robi 134201; Ibid., 26.05.2015, S. Resmi \& A.P. Janeesha 137618; Ibid., 26.05.2015, A.P. Janeesha, P.K. Dilna \& Santhosh Nampy 137620; Kakkayam-Ghat road side, 03.10.2016, A.P. Janeesha \& K. Haseem 137687; way to Kakkayam forest office, after $12^{\text {th }}$ turn, 19.09.2013, A.P. Janeesha, Habeeb Rahman \& S. Syam Radh 134221 (CALI); Palakkad district, Koomankundu, 18.09.1982, K. Prasannan 10991; Pulippara, 18.09.1982, T. Sabu 10999 (CALI); Wayanad district, Boys town-Kannur Mananthavadi route, 15.09.2016, Shimi Cheriyan, P.K. Dilna \& A.P. Janeesha 137687 (CALI); Travancore, s.d., C.C. Calder \& M.S. Ramaswami 1312 (CAL). Tamil Nadu, Coimbatore district, Aliyar Dam, 24.08.1963, K.N. Subramanian 782 (FRC); Anamalai, 04.11.1897, s.coll. s.n.; Bolamapatty hills, s.d., s.coll. $14019(\mathrm{MH})$; Dindigul district, Kodaikanal hills, 14.10.1919, K.C. Jacob 16114 (MH); Kodaikanal-Pulney, 20.10.1898, A.G. Bourne 193 (CAL); Perumal malai, 24.10.1977, M. Chandrabose 51666; Poombarai, 18.10.1977, M. Chandrabose 51365 (CAL, MH); way to Pannaikadu, 15.1968, s.coll. 30849 (MH); Kanyakumari district, Kurathividuthi estate, 11.11.1989, S.R. Srinivasan 86969 (MH); Madurai district, Anna-Manalur, 06.08.1985, K.M. Matthew 41821; Dolphin's nose, 07.07.1959, K.M. Matthew 433; Ibid., 31.10.1985, K.M. Matthew 42477 (RHT); Nilgiri district, Coonoor, 27.07.1957, K.M. Sebastine 4060 (MH); Ramanthapuram district, Deviar estate, 11.06.1979, S.R. Srinivasan 63518 (CAL, MH); Virudhunagar district, Mysore medu, 22.10.1988, S.R. Srinivasan 
89452; s.loc., 11.11.1989, S.R. Srinivasan 86972 (MH). s.loc., 20.05.1913, s.coll.691; s.loc., 23.08, s.coll. 229; s.loc., s.d., s.coll. 1784 (CAL).

Conservation status: This species is widely distributed in Kerala and Tamil Nadu and in Sri Lanka and we consider it as of Least Concern (LC) according to IUCN (2019) criteria.

Notes: This species is close to $H$. bracteata and $H$. meeboldii in leaf shape, but can be easily distinguished by its linear to oblong bracts, linear calyx lobes and hairy connectives.

Henckelia incana (Vahl) Spreng., Syst. Veg. 1: 38. 1824; Pallith., Fl. Sirumalai hills 180. 2001; T.S.Nayar et al., Fl. Pl. Western Ghats 1: 528. 2014. Roettlera incana Vahl, Enum. Pl. 1: 88. 1804. Didymocarpus rottlerianus Wall., Numer. List 778. 1829. Type: INDIA, Madurai, s.d., Röttler (probably lecto C [C10012757 digital image!]; a photograph of the type from $\mathrm{C}$ is available at E [E00155176 photograph E!]).

Didymocarpus tomentosus Wight, Icon. Pl. Ind. Orient. 4: t. 1349. 1848; C.B.Clarke in Hook.f., Fl. Brit. India 4: 353. 1885[“1884”], excluding var. lanuginosa; Rama Rao, Fl. Pl. Travancore 295. 1914; Gamble, Fl. Madras 989. 1924; S.V.Ramaswamy \& Razi, Fl. Bangalore Dist. 545. 1973; Vajr., Fl. Palghat Dist. 332. 1981; K.M.Matthew, Material Fl. Tamil Nadu 287. 1981; A.N.Henry et al., Fl. Tamil Nadu Ind., Ser I: Analysis 2: 133. 1987; K.M.Matthew, Excursion Fl. Central Tamil Nadu 359; M.Mohanan \& A.N.Henry, Fl. Thiruvananthapuram Dist. 338. 1994; Pull. \& Ali Moulali, Fl. Andhra Pradesh 2: 680. 1997; Sasidh., Biodivers. Doc. Kerala Part 6: Fl. Pl. 332. 2004; T.S.Nayar et al., Fl. Pl. Kerala 343. 2006; Pull. et al., Fl. Eastern Ghats 4: 636. 2011. Didymocarpus rottlerianus Wall. ex R.Br. var. tomentosus (Wight) C.B.Clarke in A.DC. \& C.DC., Monogr. Phan. 5: 101. 1883. Lectotype (first-step designated by Vitek et al., 2000; second-step designated here): INDIA, Tamil Nadu, Jamalay near Coimbatore, 11.1847, Wight 2352 (K [K000858196 digital image!]; isolecto K [K000858193, K000858195 digital images!]).

Fig. 8
Vernacular name: Elichuzhien (Malayalam).

Perennials, rosette-forming hairy herbs with rootstocks. Roots adventitious, thin. Leaves 6-18, basal, usually in whorls of three, rarely two; petioles 0.8-3 cm long, pubescent, winged; wings 2.3-3.4 $\mathrm{mm}$ broad; laminae elliptic-widely ovate, 5.5-11 $\times$ 3-9 cm, acute to obtuse at apex, attenuate at base, margins shallowly crenate, ultimate segments regular, surfaces usually rugose, pilose on the upper surface, tomentose on lower surface, but more so on the younger leaves and on lower veins, becoming less so with age; veins usually 5 on each side, much branched and conspicuous beneath. Cymes axillary, dense, 2-6 per plant, 2-4 times divided, 8-17-flowered, pubescent; peduncles terete, 6-23 cm long, reddish brown, pubescent; bracts two at each branching, ovate to lanceolate 2.2-2.8 $\times 0.5-0.6 \mathrm{~mm}$, obtuse to narrowly acute at apex, margins crenate, hispid along the margin; pedicels 7-15 mm long, terete, narrow, glandular hairy. Flowers 10-15 × 6-10 mm. Calyx 5-partite, very deeply divided; lobes lanceolate, unequal, posterior lobe small, 3.4-4.4 × 1-1.23 mm, others $4.1-5.2 \times 1.2-1.3 \mathrm{~mm}$, slightly acute to obtuse at apex, margins entire, reddish brown, hispid. Corolla zygomorphic, ventricose, two lipped, glandular hairy outside, glabrous inside; tube 6.7-7.3 × 5.4$7.1 \mathrm{~mm}$, lilac to pale lilac with a yellow patch in the throat; lobes oblate to widely, rounded to slightly acute at apex, lilac to pale lilac, upper lip of two lobes, 3.6-4.2 × 2.7-4.5 mm, lower lip of three lobes, $4.3-4.8 \times 3.9-5.2 \mathrm{~mm}$. Stamens 2 , adnate to the base of the corolla, included; filaments 4-5.4 mm long, glabrous, rarely few (3-5) hairs towards the top, green on the top and colourless towards base; anthers reniform, 1.6-1.8 ×0.7-0.9 mm, milky white, glabrous. Staminodes 2; filaments 2.4-3.1 mm long, hyaline, glabrous; antherodes globose or not prominent, glabrous, if globose rarely with 1 or 2 hairs. Ovary ovoid to oblong, 3.8-4.3 × 1.16$1.46 \mathrm{~mm}$, green, glandular hairy; style terete, 3.23$3.86 \mathrm{~mm}$ long, green, glabrous; stigma obliquely subcapitate, $0.4-0.6 \times 0.74-1 \mathrm{~mm}$, papillate, pale green. Capsules cylindrical, straight or slightly 


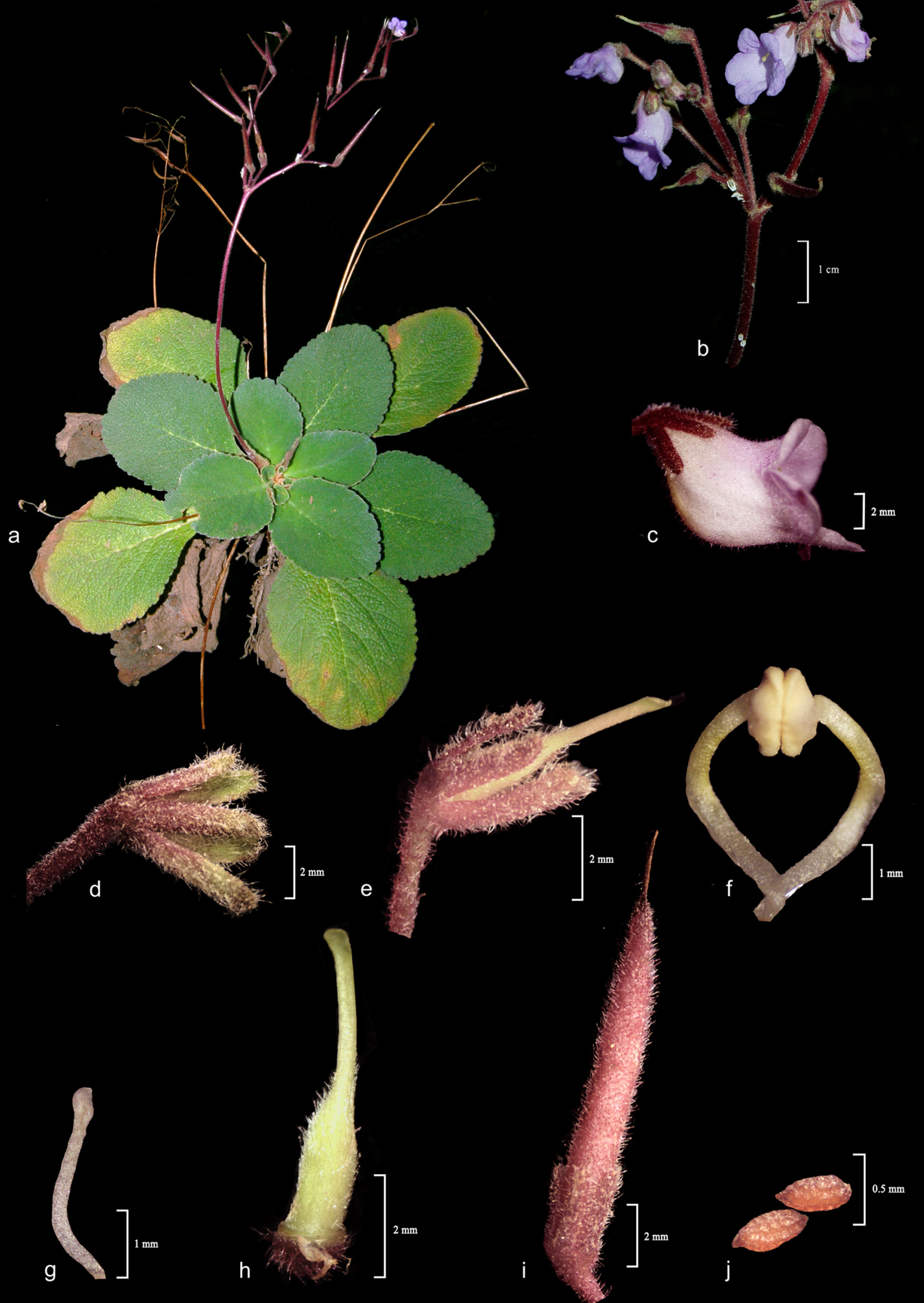

Fig. 8. Henckelia incana (Vahl) Spreng.: a. Habit; b. A portion of the inflorescence; c. Flower; d. Calyx; e. Calyx with pistil; f. Stamens; g. Staminode; h. Pistil; i. Immature capsule; j. Seeds (from A.P. Janeesha, P.M. Shahina \& K.M. Manudev 134255). 
curved, 21-28 × 2-2.4 mm, plagiocarpic, dehiscing loculicidally along the dorsal side, reddish brown, hispid, with persistent stigma and calyx. Seeds numerous, elliptic, $0.5-0.56 \times 0.22-0.26 \mathrm{~mm}$, slightly acute at apex, truncate at base; testa dark brown to black, reticulate.

Chromosome numbers: $n=27$ (Thathachar, 1942), $n$ $= \pm 45,2 n= \pm 90$ (Ratter \& Prentice, 1967).

Flowering \& fruiting: Flowering from May to October and fruiting from July to December.

Habitat: On moist slopes and in rock crevices, together with Begonia floccifera Bedd. (Begoniaceae), Justicia japonica Thunb. (Acanthaceae), species of moss and grass.

Distribution: Endemic to the southern Western Ghats (Fig. 11).

Specimens examined: INDIA, Karnataka, Bangalore district, Savandurga hilltop, 14.08.1979, K.P. Sreenath 8954 (CAL); Mysore district, Devagiri betta-Beduguli, 08.09.1961, Seshagiri Rao Rolla 73794; Ibid., 17.04.1962, A.S. Rao 80020 (E, CAL). Kerala, Idukki district, Alampetty, Marayur, 30.07.1982, V.P.K. Nambiar \& N. Sasidharan 2168 (KFRI digital image); Calvary mount, 13.11.1981, V.S. Raju 71239 (CAL); Devikulam, 14.07.1993, A. Nazarudeen 17834 (TBGT); Idukki dam, 24.08.1981, V.S. Raju 71172; Kattappana, 23.08.1977, K. Vivekananthan 50486; Ibid., 23.08.1981, V.S. Raju 71162; Ibid., 27.09.1981, C.N. Mohanan 72013 (MH); Kulamavu, 12.10.1982, C.N. Mohanan 74583 (CAL, MH); Marakkanam, 23.08.1977, K. Vivekananthan 50486 (CAL); Munnar forest division, Suryanelli, 19.10.1986, B. Gurudev Singh \& K.R. Sasidharan 12427 (FRC); on the hill between Cheruthoni and Idukki dam, 24.08.1981, V.S. Raju 71172 (CAL); way to Calvary mount, 13.11.1981, V.S. Raju s.n. (MH); Kollam district, Aryankavu, 08.06.1977, E. Sarada Amma 21894 (CALI); Kazhuthuruthy-Thenmala, 20.12.1978, C.N. Mohanan 59572; Ottakkal hills near Arnarkkad, 23.12.1969, M.V. Viswanathan MVV307 (MH); Thenmala, 13.07.2010, Geethakumary 69602 (TBGT); Kottayam district,
Kumarakam, 25.12.1980, A.S. Suletha 92205 (DEV); Palakkad district, Nelliyampathy, 16.09.1998, K. Radhakrishnan 29060 (TBGT); Ibid., 2016, P. Soumya \& A.P. Janeesha 137691; Parambikulam, 20.04.1976, Rugmini Devi 12129; Ibid., 28.04.1976, M.P. Ramani s.n.; Ibid., 26.05.2014, A. Kabeer \& A.P. Janeesha 134262 (CALI); Walayar forest, 10.07.1963, J. Joseph 17049 (E, $\mathrm{MH})$; Thiruvananthapuaram district, Arivikarai, 10.10.1928, V. Narayanaswami 934 (MH); Ponmudi, 19.09.1968, K.M. Matthew 9190; Ibid., 12.09.1970, K.M. Matthew 12411 (RHT); Ibid., 16.08.1980, M. Mohanan 69225; Ibid., 17.11.1977, M. Mohanan 52549; Ibid., 25.05.1979, M. Mohanan 63280 (MH); Ibid., s.d., M. Abdul Jabbar 13777; Ibid., 29.10.1983, Satheesh Kumar 5921 (TBGT); Ibid., 28.11.2012, A.P. Janeesha, P.M. Shahina \& K.M. Manudev 134255; Ibid., 31.07.2014, Santhosh Nampy \& A.P. Janeesha 134146, 134148; Ibid., 31.07.2014, S. Syam Radh, Santhosh Nampy \& A.P. Janeesha 134152, 134165, 134168 (CALI); Ponmudi-way to upper sanatorium, 25.05.1979, M. Mohanan 63280; Ibid., 16.08.1980, M. Mohanan 69225 (CAL); Wayanad district, Edakkal cave-inside, 08.07.2016, A.P. Janeesha \& P.G. Arunkumar 137674 (CALI). Tamil Nadu, Coimbatore district, Aliyar submergible area, 28.07.1962, K.M. Sebastine 14686 (CAL, MH); Anamalai, 08.10.1901, C.A. Barber 3629; Ibid., 10.10.1901, C.A. Barber 3659 (MH); Ibid., 26.06.1973, R.N. Kajal \& G.N. Tribedi 567 (CAL); Andiparai shola R.F., 26.06.1973, E. Vajravelu $44302(\mathrm{MH})$; around Attakatti, s.d., J. Joseph 12685 (CAL, MH); Bolampetty valley, 24.02.1917, C.E.C. Fischer 4059 (CAL); Hassanur, 25.08.1914, s.coll. 10634; Kurudimalai, 27.09.1956, K. Subramanyam 865; Ibid., 17.11.1968, J.L. Ellis \& S. Karthikeyan 31325; Ibid., 20.01.1970, M.V. Viswanathan MVV375 (MH); Kuridimalainorthern slopes, 27.09.1956, K. Subramanyam 865 (CAL); Marudamalai, 24.06.1930, V. Narayanaswami 3072 (MH); Ibid., 09.08.2014, K. Thoiba \& A.P. Janeesha 134280; ValparaiShivamalai, 13.12.2013, A.P. Janeesha \& Santhosh Nampy 134244; Velliangiri hills-way to temple, 29.05.2014, S. Syam Radh, R. Rajeevan \& A.P. 
Janeesha 138187, 138189, 138191; Ibid., 30.09.2016, Nikhil Krishna \& A.P. Janeesha 137689 (CALI); Dindigul district, Kodaikanal, 20.11.2012, K.M. Manudev, A.P. Janeesha \& Santhosh Nampy 134254 (CALI); Perumparai, 22.10.1977, M. Chandrabose 51612 (CAL); Ratter water fall, 25.02.2014, A. Kabeer, K. Shinoj \& A.P. Janeesha 134258 (CALI); Sirumalai, 16.05.1958, J.M. Pallithanam 3356 (BLAT); Kanyakumari district, Kooladai, 08.09.1969, B.V. Shetty 32293; Maranthuvalmalai, 18.10.1985, R. Gopalan 83374; Sevgaltai, 24.09.1915, s.coll. 12447 (MH); Thekkumalai, 19.11.1956, K.M Sebastine 1368 (CAL, MH); Madurai district, Amaravathi sagaram-lower camp, 22.06.1968, D.B. Deb 30121; Cumbum valley, 20.06.1959, K. Subramanyam 8048 (MH); High way hut, 05.1917, E. Blatter \& Hallberg 355 (BLAT, CAL); Sirumalai, 23.08.1913, C.A. Barber 9058 (MH); Vengayapparai, 21.11.1985, K.M. Matthew, A. Usha \& N. Rajendran 42987 (RHT); way to Perumparai, 22.10.1977, M. Chandrabose 51612 (MH); Namakkal district, Kolli hills, 01.08.1978, N. Venugopal 15984 (CAL); Nilgiris district, Bimaka shola, 26.08.1970, G.V. Subba Rao 36346 (MH); Kil Kotagiri-on the way, 25.10.1956, K. Subramanyan 1171 (CAL); Kodanad view point, 24.07.1970, E. Vajravelu 35084 (MH); Mudumalai, 03.06.1994, S.K. Mandal 7214 (CAL); Naduvattam, 28.06.1970, B.V. Shetty 34457; Ibid., 30.08.1970, B.D. Sharma 35926; way to Anakkatai from Ebanad, 27.07.1972, G.V. Subba Rao 41563; way to Kilkotagiri, 25.10.1972, K. Subramanyam 1171 (MH); way to Naduvattam from Devar shola, 21.07.1960, K. Subramanyam 10517 (CAL, MH); Pykara falls, 08.1884, J.S. Gamble 15292 (CAL, BM digital image); Ibid., 06.1884, J.S. Gamble 14241 (BM digital image); Pudukkottai district, Ponnamaravathi, 22.07.1984, C. Arulappan s.n. (MH); Ramnad district, Kendiparai-Ayyanar koil, 23.09.1971, E. Vajravelu 38704; Mudaliarattu, 21.09.1917, P.S. Jivanna Rao 15096 (MH); Salem district, Kolli hills, 10.09.1992, A.A. Ansari 97066; Retreat area-Yercaud, 24.07.1965, S. Karthikeyan 26853 (MH); Thiruchirapalli district, Trichy, 18.10.1985, P.C. Jose Kutty 1146 (DEV); Tenkasi district, Courtallam, 04.07.1974, T.N. Reetha Bai 30815 (CALI); Tirunelveli district, Chunkankada hills, near Sree Ayyappa College, 20.07.2016, A.P. Janeesha, S. Resmi \& P.G. Arunkumar 137676 (CALI); Karayar Dam, 30.07.1989, Shanavas Khan 5853 (TBGT); Kohimalai, 02.11.1914, s.coll. 11396; Kulurathumottai grassland, 16.10.1992, R. Gopalan 9997; Mahendragiri, 17.09.1916, s.coll.13139 (MH); near Kodayar power house, 19.07.2016, A.P. Janeesha, S. Resmi \& P.G. Arunkumar 137675 (CALI); Neelikkathu R.F., 03.05.1989, R. Gopalan 90532 (MH); Papanasam project, 07.11.1959, K.M. Sebastian 9560 (CAL); Thulukkam parai, 28.11.1969, B.V. Shetty 32947; Vasudevanallur R.F., 03.10.1971, E. Vajravelu 38847 (MH); Vellore district, slopes of Konamalai, 18.07.1961, C.P. Sreemadhavan CPS741; s.loc., 27.09.1986, S.R. Raja \& Nagavath s.n. (MH). State unknown, s.loc., 06.1883, J.S. Gamble 11780 (CAL). Peninsular India, s.d., R. Wight 2352 (CAL).

Conservation status: This species is widely distributed in Karnataka, Kerala and Tamil Nadu and the thresholds for Extent of Occurrence (EOO) and Area of Occupancy (AOO) are not met by this species to fall into one of the threat categories. We therefore consider it as of Least Concern (LC) according to IUCN (2019) criteria.

Notes: Wallich (1829) in his catalogue listed Didymocarpus rottlerianus and quoted Roettlera incana as a synonym. D. rottlerianus Wall. is thus validated by Roettlera incana and became nomenclaturally superfluous (for more information, see Burtt, 1954) and both names have Rottler's specimen as type.

Typification: Vahl (1804) described Roettlera incana (H. incana (Vahl) Spreng.) based on Röttler's specimen from mountains in Madurai, India. Vitek et al. (2000) cited the sheet at C (C10012757 digital image!) as the holotype. A further search revealed a photograph of the above sheet from $\mathrm{C}$ at $\mathrm{E}$ with the barcode E00155176 (photograph E!). The sheet C10012757 contains two flowering specimens mounted separately but do not bear any field label or collector's information to ascertain it as a type material. A further search revealed one sheet with 
two barcodes at K (K001129365, K001111886 digital images!) collected by Röttler. Except the one specimen mounted on the upper side of the sheet, all others agree with $H$. incana. However, the field label attached at the top of the sheet indicated as locality "ooty". Hence, this specimen can also not be considered for typification. According to Stafleu and Cowan (1983), Röttler's main collections are kept at $\mathrm{K}$ and LIV and other materials are at C, ER, FI, H, H-Aeh, JE, LE, M, MH, MO, MW and NY. Even though a lectotypification is necessary, access to some herbaria material has been impossible in time, lectotypification is postponed here and will be carried out in a separate paper.

Wight (1848) described Didymocarpus tomentosus Wight and included an illustration of his collection from Kaitie falls, on the Neilgherries. It was further mentioned in the protologue "I have since received many specimens from hills near Coimbatore, and have often met with it in similar situations". All of this is original material and available for lectotypification. Vitek et al. (2000) cited "Wight (K)" as the type. We traced three relevant specimens at K (K000858193, K000858195, K000858196 digital images!). One sheet K000858193 with an HRWP label is annotated "Did. tomentosa?, Jamalay Coimbatore" by Wight. The sheets K000858195 and K000858196 have the annotations "2352 Didymocarpus tomentosus Wt", with field labels, one with "Jamalay, June 1848" and the other "Jamalay near Coimbatore, November 1847" respectively. Specimens from Kaitie falls are, however, not available in any herbaria. The sheet K000858196, earliest among them with five mature plants and scapes is chosen here as the second-step lectotype while the others (K000858193, K000858195) are consequently isolectotypes.

Henckelia innominata (B.L.Burtt) A.Weber \& B.L.Burtt, Beitr. Biol. Pflanzen 70: 347. 1998[“1997"]; T.S.Nayar et al., Fl. Pl. Western Ghats 1: 528. 2014. Didymocarpus innominatus B.L.Burtt, Notes Roy. Bot. Gard. Edinburgh 21: 201. 1954; B.D.Sharma et al., Fl. Karnataka Analysis 195. 1984; A.N.Henry et al., Fl. Tamil Nadu Ind., Ser I: Analysis 2: 132. 1987; M.Ahmedullah \&
M.P.Nayar, Endemic Pl. Indian Region 1: 143. 1987; N.P.Singh, Fl. Eastern Karnataka 2: 475. 1988; Pull. \& N.Yesoda, Fl. Anantapur Dist. 182. 1989; Sasidh. \& Sivar., Fl. Pl. Thrissur Forest 329. 1996; Pull. \& Ali Moulali, Fl. Andhra Pradesh 2: 679. 1997; Sasidh., Biodivers. Doc. Kerala Part 6: Fl. Pl. 332. 2004; T.S.Nayar et al., Fl. Pl. Kerala 342. 2006; Pull. et al., Fl. Eastern Ghats 4: 361. 2011. Lectotype (first-step designated by Vitek et al., 2000; second-step designated here): s.loc., s.d., Wallich 778 (K [K001111885 (778B) digital image!]; isolecto K [K00085184, K001111884 digital images!]).

Figs. $9 \& 10$

Perennials, rosette-forming hairy herbs with rootstocks. Roots adventitious, thin. Leaves 6-15, basal, usually in whorls of three; petioles $2.5-9 \mathrm{~cm}$ long, pubescent, winged; wings $1-3 \mathrm{~mm}$ broad; laminae elliptic, 8.5-12 × 4.7-6.7 cm, acute at apex, narrowly cuneate to attenuate at base, margins doubly serrate, first serration deep and ultimate segments irregular, surfaces usually rugose; hispid on both surfaces, but more so on the younger leaves and on lower veins, becoming less so with age, veins usually 5 on each side, much branched and conspicuous beneath. Cymes axillary, 2-7 per plant, 3-5 times divided, 12-22-flowered, pubescent; peduncles terete, $18-24 \mathrm{~cm}$ long, green, pubescent; bracts two at each branching point, linear, 3-5 $\times$ 1.3-2.4 mm, obtuse at apex, margins entire, hispid; pedicels terete, 4-14 $\mathrm{mm}$ long, narrow, pubescent. Flowers 10-20 × 4-9 mm. Calyx 5-partite, very deeply divided or basally connate; lobes elliptic to oblong, unequal, posterior lobe small, 2-2.2 ×0.68$0.77 \mathrm{~mm}$, others $2.3-2.9 \times 0.71-1 \mathrm{~mm}$, obtuse at apex, margins entire, reddish brown towards the base and green towards the apex, hispid on both sides. Corolla zygomorphic, ventricose, two lipped, glandular hairy outside, glabrous inside; tube $6.8-$ $7.9 \times 5.3-6.2 \mathrm{~mm}$, pale lilac with a yellow patch in the throat; lobes widely ovate to orbicular, rounded at apex, slightly undulate, pale lilac, upper lip of two lobes, $4.5-5.2 \times 5.3-5.9 \mathrm{~mm}$, lower lip of three lobes, 5.4-5.8 × 5.3-6.2 mm. Stamens 2, adnate to the base of the corolla, included; filaments 3.3-3.8 $\mathrm{mm}$ long, glabrous, green on the top and colourless 

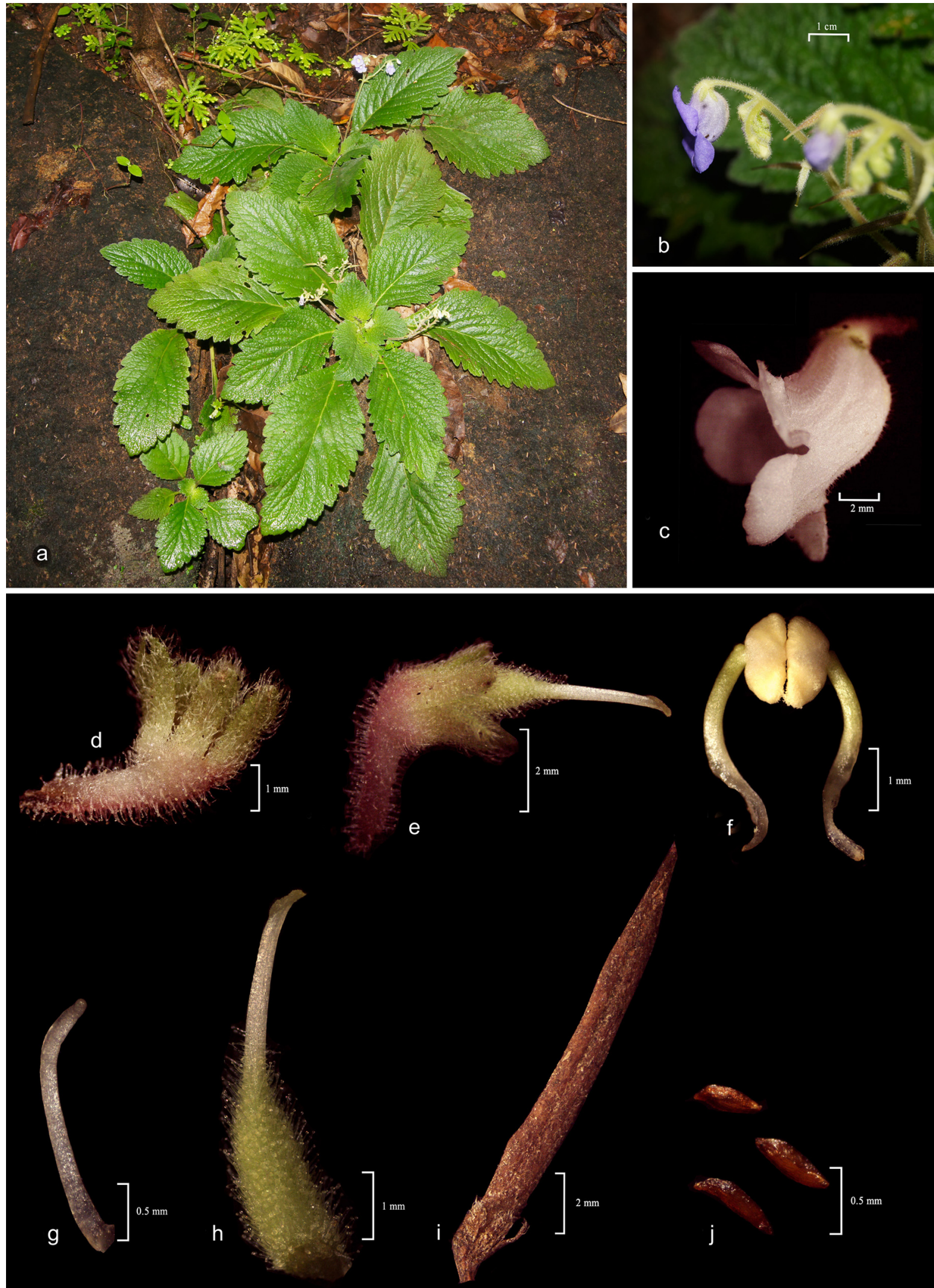


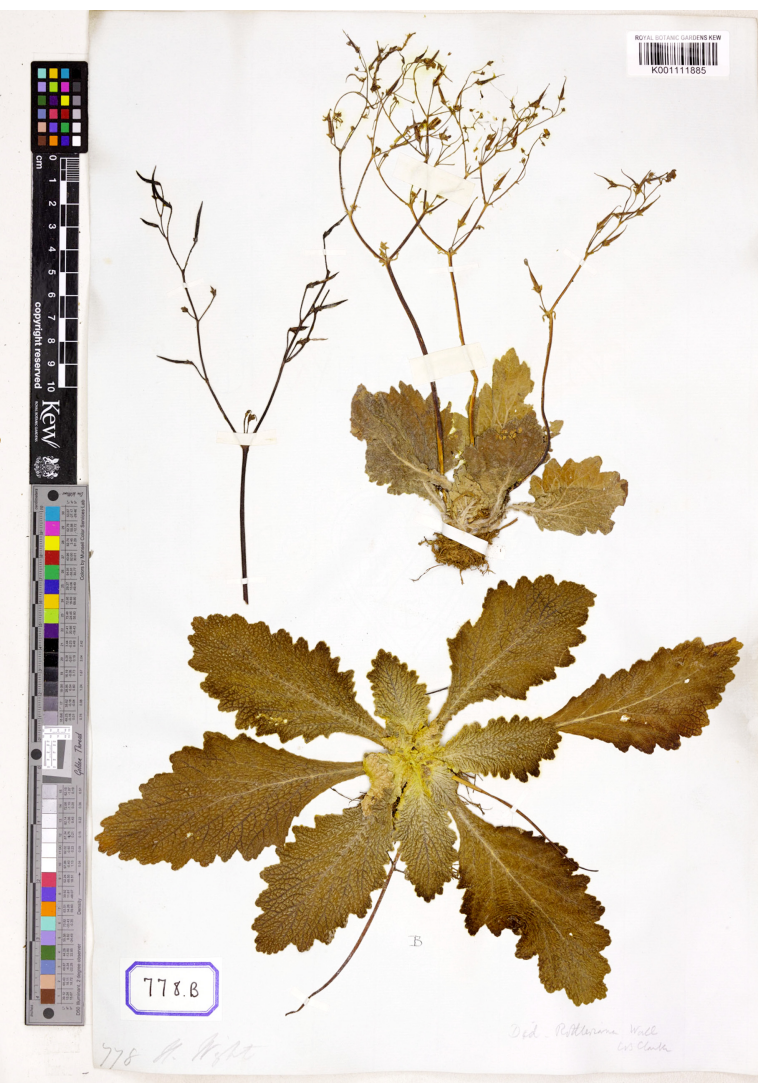

Fig. 10. Lectotype of Henckelia innominata (B.L.Burtt) A.Weber \& B.L.Burtt. (C) The Board of Trustees for The Royal Botanic Gardens, Kew. Reproduced with permission.

towards base; anthers reniform, 1.45-1.6 × 0.62$0.7 \mathrm{~mm}$, milky white, glabrous. Staminodes 2; filaments $1.8-2 \mathrm{~mm}$ long, hyaline, glabrous; antherodes a small knob like structure. Ovary ovoid to oblong, 3.1-3.5 × 1.15-1.3 mm, green, glandular hairy; style terete, 2.4-2.6 mm long, green, glabrous; stigma obliquely subcapitate, $0.53-0.58$ $\times 0.3 \mathrm{~mm}$, papillate, pale green. Capsules cylindrical, straight, $12-19 \times 1.4-1.6 \mathrm{~mm}$, plagiocarpic, dehiscing loculicidally along the dorsal side, reddish brown, tomentulose with persistent stigma and calyx. Seeds numerous, elliptic, 0.43-0.56 ×0.2 mm, slightly acute at both ends; testa dark brown to black, reticulate.

Chromosome numbers: $n=16,2 n=32$ (Ratter \& Prentice, 1967).

Flowering \& fruiting: Flowering from (May) June to September and fruiting from August to December.
Habitat: On moist slopes and in rock crevices, along with Selaginella sp. (Selaginellaceae), Spermacoce alata Aubl. (Rubiaceae) and a few grass species.

Distribution: Endemic to the southern Western Ghats (Fig. 11).

Specimens examined: INDIA, Karnataka, Coorg district, s.loc., s.d., s.coll. s.n. (CAL). Kerala, Idukki district, Chinnar, 19.01.1986, B. Gurudev Singh \& K.R. Sasidharan 12428 (FRC); Ibid., 07.10.1994, E.S. Santhosh Kumar 17595 (TBGT); Kollam district, Achencoil, 22.05.1979, C.N. Mohanan 63025 (MH); Moonnamra-Thenmala, 17.08.2016, A.P. Janeesha \& Santhosh Nampy 137681; Moonnamra-near constructing railway track, 17.08.2016, A.P. Janeesha, S. Resmi, Dani Francis \& K.S. Arathy 137682 (CALI); Palakkad district, Dhoni reserve forest, 20.07.1963, J. Joseph 17220 (MH); Pathanamthitta district, Achankovil to Mekkarai, 05.09.1913, M. Rama Rao 1652 (CAL). Tamil Nadu, Coimbatore district, Attakatti, 04.07.1961, J. Joseph 12685 (MH); Ibid., 21.11.1980, M. Chandrabose \& V. Chandrasekaran 69044 (MH, CAL); Parappalar dam, 11.11.1986, K.M. Matthew 47293 (RHT); Siruvani, 14.08.1960, A.N. Henry 351 (MH); Siruvani-near Bungalow, 14.08.1960, A.N. Henry 350 (BLAT). Dharmapuri district, Chitteri hills, 12.08.1978, K.M. Matthew 16362; Ibid., 09.01.1980, K.M. Matthew 25618 (RHT); Kanyakumari district, s.loc., 29.10.2015, K. Thoiba \& A.P. Janeesha 137651, 137652 (CALI); Nilgiri district, s.loc., s.d., G. Thomson 1351 (CAL); Ramnad district, Ayyanarkoil, 23.09.1971, E. Vajravelu 38715; Cumbummedu, 14.03.1970, E. Vajravelu 33767; Yanaimutti rocks, 13.12.1972, E. Vajravelu 39412 (MH); Salem district, Krishnagiri, 24.09.1917, s.coll. 14935 (MH); Yercaud, 28.06.1980, V. Sainaba 28364 (CALI); Tenkasi district, Courtallam, 18.07.1901, C.A. Barber 3374; Ibid., 16.07.1907, C.A. Barber 8378; Ibid., 23.07.1957, K. Subramanyam 3745 (MH); Ibid., 29.06.1980, K. Indira Devi 5760; Ibid., 30.06.1980, K.S. Hema 8143; Ibid., 30.06.1980, T. Usha 30144; Ibid., 30.06.1980, M.S. Baby Usha Kiran 3783 (CALI); Tirunelveli district, around Shembagadevi, 27.09.1975, K.K.N. 


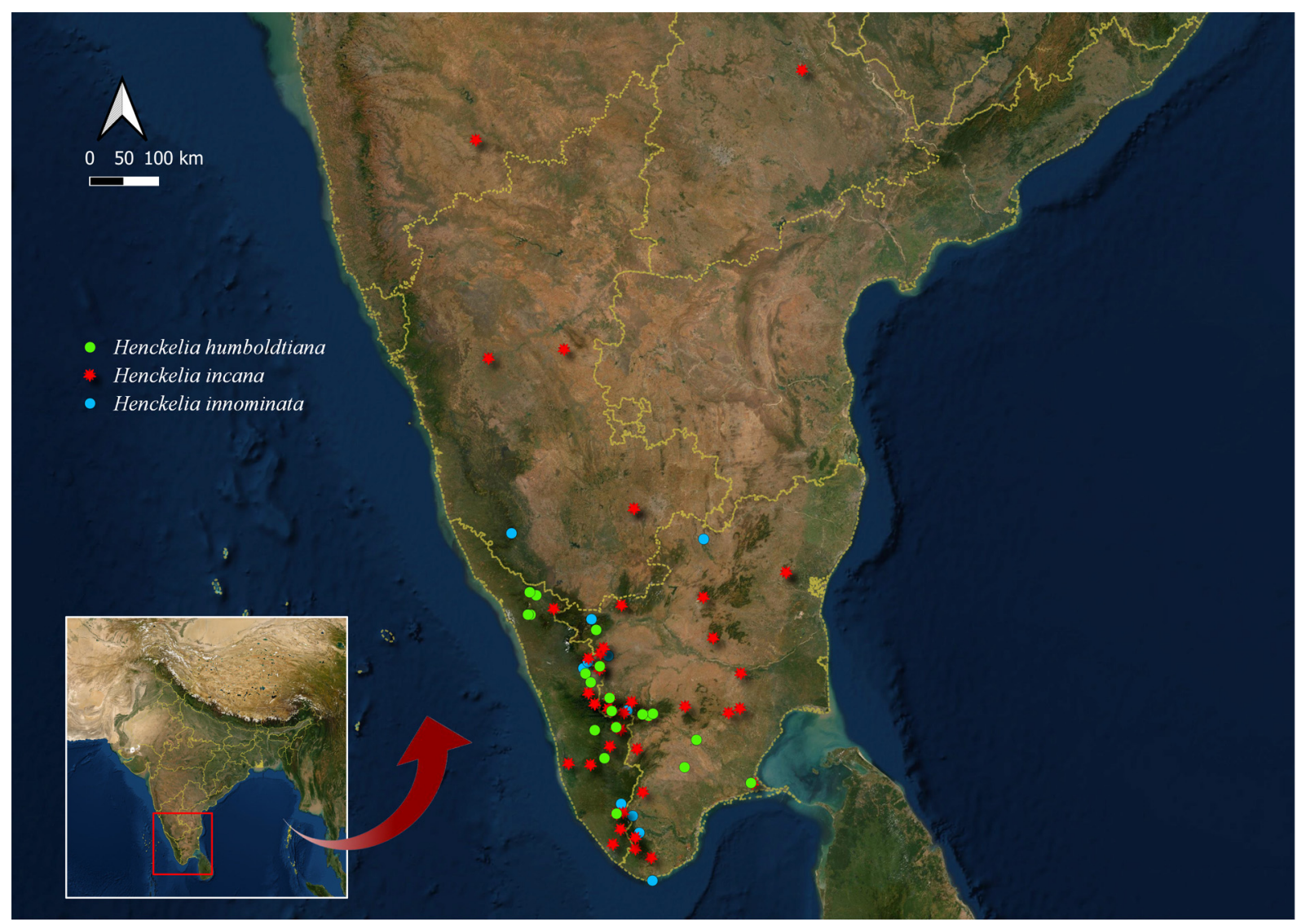

Fig. 11. Distribution of Henckelia humboldtiana (Gardner) A.Weber \& B.L.Burtt, H. incana (Vahl) Spreng. and H. innominata (B.L.Burtt) A.Weber \& B.L.Burtt in South India (drawn using QGIS v.3.12.1).

Nair 1167 (CAL); forest near Papanasam project, 07.11.1959, K.M. Sebastine 9560; Kannakattii, 13.06.1899, C.A. Barber 505 (MH); Mekkarai-on rock, 06.09.1913, C.C. Calder \& M.S. Ramaswami 620 (CAL); Naterikal, 21.09.1914, s.coll. 10952 (MH); Sengalten, 16.02.1913, D. Hooper \& M.S. Ramaswami 39219 (CAL); Vasudevanallur, 13.11.1925, S.R. Raju 17801; Ibid., 03.10.1971, E. Vajravelu 38845 (MH); s.loc., 01.09.1913, C.C. Calder \& M.S. Ramaswami 507. s.loc., 01.06.1905, C.E.C. Fischer 22; s.loc., s.d., M.S. Ramaswami 607; s.loc., s.d., s.coll. 67; s.loc., s.d., R. Wight 2678 (CAL, E); s.loc., s.d., s.coll. 778 (CAL).

Conservation status: The species occurs widely in 13 districts in three states, Karnataka, Kerala and Tamil Nadu. The Extent of Occurrence (EOO) is estimated to be $c .34100 \mathrm{~km}^{2}$. However, during field surveys of two localities, only 106 mature plants were observed, suggesting that the populations are not large. Not all of the localities are in protected areas and those outside face high risks of habitat destruction, particularly one that is located very near to a railway track construction site. However, because of the number of populations and localities, the species can be considered at present of Least Concern (LC) according to IUCN (2019) criteria.

Typification: Wallich (1829) in his catalogue named this species Didymocarpus rottlerianus Wall. but quoted Roettlera incana Vahl as its synonym. The name $D$. rottlerianus thus got validated and became nomenclaturally superfluous (for more information, see Burtt, 1954). Burtt (1954) named Wallich's specimen 778 as D. innominatus B.L.Burtt. Vitek et al. (2000) cited "Wallich 778 [K]" as the type. We have traced five Wallichian collections at $\mathrm{K}$ (K001129365, K00085184, K001111885, K001111884, K001111886 digital images!). In order to narrow down the choice to a single specimen, we carried out a second-step lectotypification here. The sheets K001129365 (labelled 777C \& D) and 
K001111886 are mixed collections, and the specimens mounted on the upper side alone belong to H. innominata. K00085184, K001111885, K001111884 are labelled 778, 778B \& 778.1 respectively and all belong to $H$. innominata. Of these, sheet K001111885 with a complete plant and inflorescence is chosen here as the lectotype while the other specimens (K00085184, K001111884) are consequently isolectotypes.

Henckelia lyrata (Wight) A.Weber \& B.L.Burtt, Beitr. Biol. Pflanzen 70: 349. 1998[“1997”]; T.S.Nayar et al., Fl. Pl. Western Ghats 1: 528. 2014; Janeesha \& Nampy, Phytotaxa 268(1): 80. 2016; M.P.Geethakumary et al., Phytotaxa 284(2): 147. 2016. Didymocarpus lyratus Wight, Icon. Pl. Ind. Orient. 4: t. 1350. 1848; C.B.Clarke in Hook.f., Fl. Brit. India 4: 353. 1885["1884"]; Rama Rao, Fl. Pl.Travancore 295. 1914; Gamble, Fl. Madras 2: 988. 1924; A.N.Henry et al., Fl. Tamil Nadu Ind., Ser I: Analysis 2: 132. 1987; M.Ahmedullah \& M.P.Nayar, Endemic Pl. Indian region 1: 143. 1987; Pull. et al., Fl. Eastern Ghats 4: 361. 2011. Roettlera lyrata (Wight) Kuntze, Revis. Gen. Pl. 2: 476. 1891. Lectotype (designated by Janeesha \& Nampy, 2016): INDIA, Tamil Nadu, Courtallam, 09.1835, Wight 559 (E [E00155173 digital image!]; isolecto E [E00155174, E00155173 digital images!], K [K000858187 digital image!]).

Didymocarpus lyratus Wight var. protractus C.B.Clarke in A.DC. \& C.DC., Monogr. Phan. 5: 102. 1883, syn. nov.; A.N.Henry et al., Fl. Tamil Nadu Ind., Ser I: Analysis 2: 132. 1987. Lectotype (designated by Vitek et al., 2000): INDIA, Pulney hills, Beddome 8505 (BM).

Fig. 12

Perennials, rosette-forming hairy herbs with rootstocks. Roots adventitious, thin. Leaves 6-9, basal, usually in whorls of three; petioles $4.5-14 \mathrm{~cm}$ long, pubescent, lacerate; laminae widely ellipticorbicular, $4.5-7.5 \times 4.5-6.3 \mathrm{~cm}$, rounded to obtuse at apex, lyrate at base, margins doubly crenate to serrate, sometimes the first crenation is very deep and irregular, surfaces usually rugose, pilose on both surfaces, but more so on the younger leaves and on lower veins, becoming less so with age; veins usually
5 on each side, much branched and conspicuous beneath. Cymes axillary, 3-9 per plant, 3-5 times divided, 12-21-flowered, pubescent; peduncles terete, 13-29 cm long, green, pubescent; bracts two at each branching point, linear, 3-5 × c. $1 \mathrm{~mm}$, obtuse to rounded at apex, margins entire, pilose; pedicels terete, 4-25 mm long, narrow, pubescent. Flowers 10-14 × 5-7 mm. Calyx 5-partite, very deeply divided; lobes broadly lanceolate, unequal, posterior lobe small, 2-4.5 × 0.5-1.3 mm, others 4-11 × 0.7-3 mm, obtuse at apex, margins entire, green, pilose. Corolla zygomorphic, ventricose, two lipped, minutely villous outside, glabrous inside; tube 8-10 × 5-6.7 mm, white to pale lilac with a yellow patch in the throat; lobes widely ovate, ovate to rounded at apex, white to pale lilac, upper lip of two lobes, 3.7-4.1 × 4-4.8 mm, lower lip of three lobes, 4-5 × 4.2-5.3 mm. Stamens 2, adnate to the base of the corolla, included; filaments $3.5-4.2 \mathrm{~mm}$ long, glabrous; anthers reniform, 1.5-1.6 × c. 0.62 mm, milky white. Staminodes 2; filaments 3.1-3.5 $\mathrm{mm}$ long, glabrous; antherodes highly reduced. Ovary ovoid to oblong, 2.6-3 × 0.78-0.86 mm, pubescent; style terete, $3-3.8 \mathrm{~mm}$ long, glabrous; stigma obliquely subcapitate, c. $0.34 \mathrm{~mm}$ diam., papillate, white to pale green. Capsules cylindrical, straight or slightly curved, 12-36 mm long, plagiocarpic, dehiscing loculicidally along the dorsal side, green, glandular hairy with persistent stigma and calyx. Seeds numerous, oblong-ovoid to elliptic, $0.4-0.51 \times 0.17-0.21 \mathrm{~mm}$, slightly acute at apex; testa dark brown to black, reticulate.

Flowering \& fruiting: Flowering from May to October and fruiting from August to December.

Habitat: On moist rocks, in association with Porella sp. (Porellaceae).

Distribution: Endemic to the southern Western Ghats (Fig. 17).

Specimens examined: INDIA, Kerala, Idukki district, Neymakkad gap, N $10^{\circ} 08246.322^{\prime}$, E $77^{\circ} 052$ 16.93', 1784 m, 22.10.2004, M.P. Geethakumary \& A.G. Pandurangan 55339 (TBGT); Kollam district, Aryankavu, 02.12.1961, K.N. Subramanian 77355 (BSI); Palakkad district, Dhoni forest-Korakkallu 

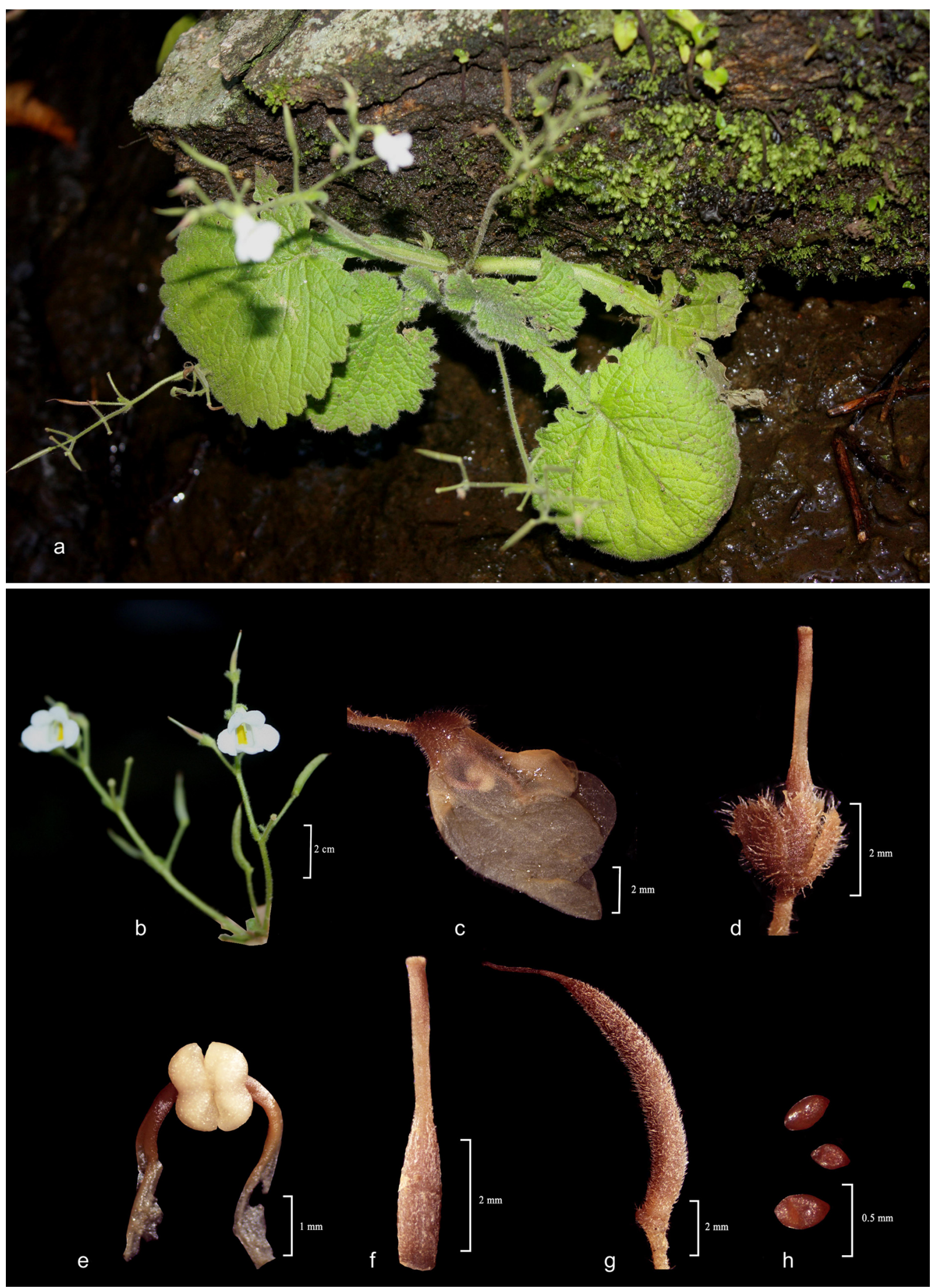

Fig. 12. Henckelia lyrata (Wight) A.Weber \& B.L.Burtt: a. Habit and habitat; b. A portion of the inflorescence; c. Flower; d. Calyx with pistil; e. Stamens; f. Pistil; g. Immature capsule; h. Seeds (from K.M. Prabhukumar 7559). 
region, 27.09.2013, K.M. Prabhukumar 7559 (CALI). Tamil Nadu, Ramnad district, Kendiparai slopes-Ayyanarkoil, 23.09.1971, E. Vajravelu 38715; Kumbumedu, 14.03.1970, E. Vajravelu 33767; Yanimutti Rock-Mudaliaruthu, 13.12.1972, E. Vajravelu 39412 (MH); Virudhunagar district, Srivilliputhur Wildlife sanctuary, 24.10.2013, Kabeer \& G. Gnanashekaran 130400 (MH).

Conservation status: The species occurs in Kerala and Tamil Nadu with around five localities in five districts, with one isolated disjunct locality in the North. The estimated Extent of Occurrence (EOO) is $c .7800 \mathrm{~km}^{2}$. The authors were able to study three localities where they found a total of 26 mature plants. Of the three populations, two populations are located in unprotected areas and one in a wildlife sanctuary. The situation at Aryankavu and Mudaliaruthu are currently unknown. With view to the location and small size of the populations a further decline is likely. Thus, we assess the species as Endangered (EN), B2ab(iii,iv,v) according to IUCN (2019) criteria.

Notes: The lyrate, pilose leaves, shorter scapes and small flowers identify this species in the field. Clarke (1883) described another variety, Didymocarpus lyratus var. protractus, differentiating from the typical variety by its elliptic, narrowly winged lyrate, less pilose, crenate leaves, $8-12 \mathrm{~cm}$ long petioles and longer peduncles (up to $24 \mathrm{~cm}$ long). But most of these characters are within the limit of H. lyrata (see description above) and hence var. protractus is treated here as conspecific.

Henckelia macrostachya (E.Barnes) A.Weber \& B.L.Burtt, Beitr. Biol. Pflanzen 70: 349. 1998 [“1997”]; Shaju et al., Taprobanica 5(2): 138. 2013; T.S.Nayar et al., Fl. Pl. Western Ghats 1: 528. 2014. Didymocarpus macrostachya E.Barnes, Bull. Misc. Inform. Kew 1938: 37. 1938; M.Ahmedullah \& M.P.Nayar, Endemic Pl. Indian Region 1: 143. 1987; Sasidh., Biodivers. Doc. Kerala Part 6: Fl. Pl. 332. 2004; T.S.Nayar et al., Fl. Pl. Kerala 342. 2006. Lectotype (designated by Vitek et al., 2000): INDIA, Travancore, high range, on Ottapparai ridge, 5500 ft., 17.09.1935, E. Barnes 1266 (K [K000858197 digital image!]; isolecto K [K000938534, K000938535 digital images!]).

Fig. 13

Perennials, rosette-forming hairy herbs with rootstocks. Roots adventitious, thin. Leaves 9-12, basal, usually in whorls of three, sessile to sub-sessile; laminae obovate-widely spatulate, $16-27 \times 6-10$ $\mathrm{cm}$, rounded to slightly acute at apex, attenuate at base, margins crenate, surfaces usually rugose, white bullate hairy on the upper surface, with dense golden brown woolly hairs on the younger leaves and on lower veins, becoming less so with age; veins 7-8 on each side, much branched and conspicuous beneath. Cymes axillary, 1-2 per plant, 3-6 times divided, 16-28-flowered; peduncles terete, 30-54 cm long, stout, golden brown, covered with dense golden brown woolly hairs; bracts two at each branching point, broadly linear to elliptic, 1.5-4 $\times$ 1-1.9 mm, rounded at apex, basally hairy, green; pedicels terete, 3-11 mm long, slender, hairy. Flowers 10-20 × 13-18 mm. Calyx 5-partite, very deeply divided or basally connate; lobes ovate to elliptic, unequal, posterior lobe small, 2-2.7 $\times 0.8-$ $1.3 \mathrm{~mm}$, others $2.8-4.9 \times 1-1.92 \mathrm{~mm}$, slightly acute to obtuse at apex, margins entire, reddish brown, glandular hairy on the base, with golden brown woolly hairs towards the apex. Corolla zygomorphic, ventricose, two lipped, with white woolly hairs outside, glabrous inside; tube 8-12 $\times$ 4.3-5.5 mm, pale lilac to white with a yellow blotch at the throat; lobes very widely obovate with undulate margin, rounded at apex, white to pale lilac, upper lip of two lobes, 5.5-6 × 7-7.8 mm, lower lip of three lobes, $6.8-7.8 \times 7.7-8.6 \mathrm{~mm}$. Stamens 2, adnate to the base of the corolla, included; filaments $4.5-5 \mathrm{~mm}$ long, glabrous, yellow; anthers reniform, 1.5-2.75 ×0.75-1.16 mm, milky white to cream, bearded in the region of the connective. Staminodes 2; filaments $2.4-3.3 \mathrm{~mm}$ long, hyaline, glabrous; antherodes dome shaped, 0.4-0.62 × 0.5-0.7 mm, bearded in the region of connective. Ovary ovoid, 4-6 × 2.1-2.64 mm, pale green, with glandular and dense white woolly hairs all over; style terete, 1.8-3 mm long, green, glabrous; stigma obliquely subcapitate, $0.65-0.7 \times 0.8-0.86$ $\mathrm{mm}$, papillate, pale green. Capsules cylindrical, stout, 

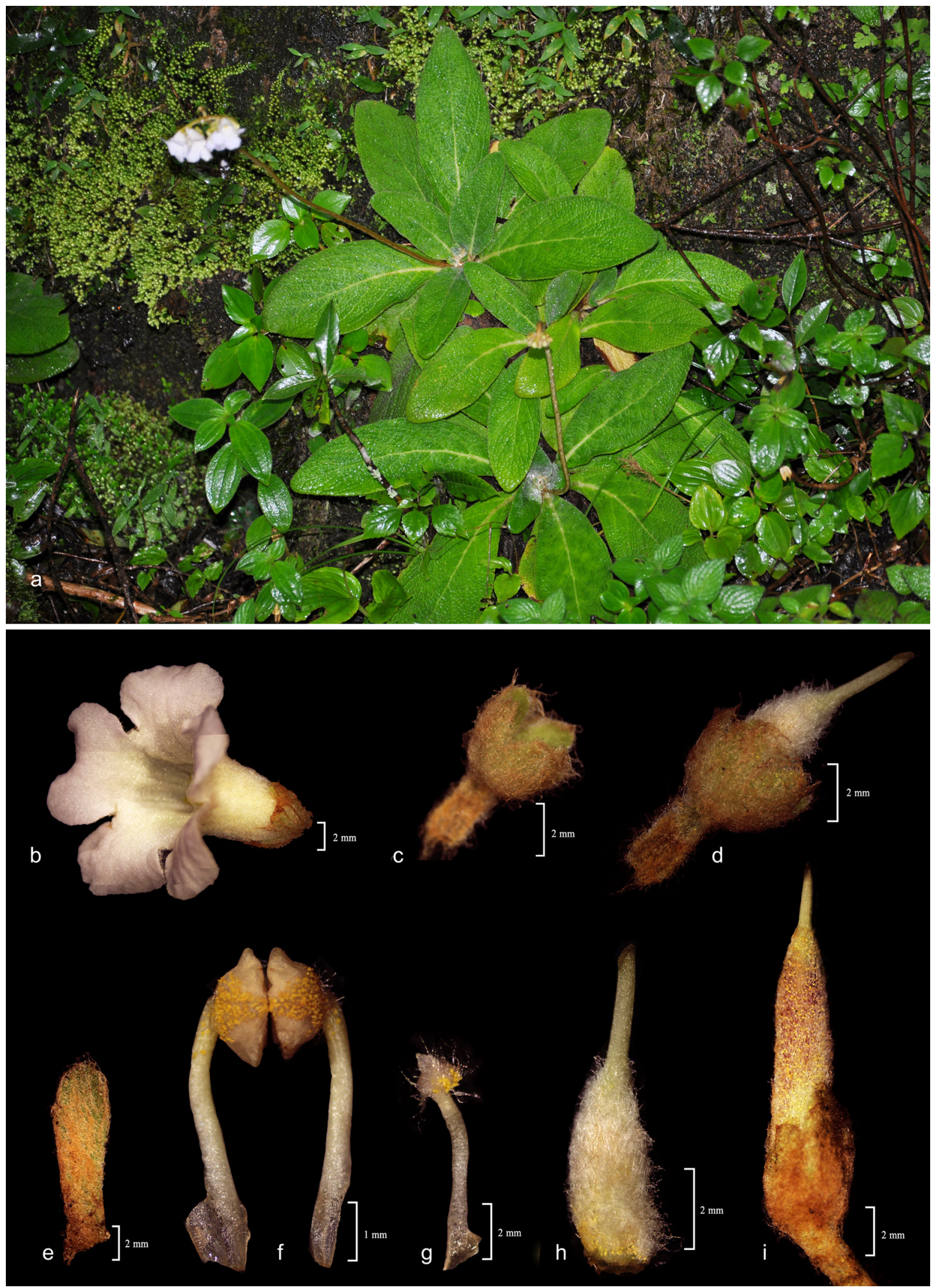

Fig. 13. Henckelia macrostachya (E.Barnes) A.Weber \& B.L.Burtt: a. Habit and habitat; b. Flower; c. Calyx; d. Calyx with pistil; e. Bract; f. Stamens; g. Staminode; h. Pistil; i. Immature capsule (from A.P. Janeesha \& Santhosh Nampy 137616). 
13-28 × 2.2-2.7 mm, plagiocarpic, dehiscing loculicidally along the dorsal side, reddish brown, glandular hairy with persistent stigma and calyx. Seeds numerous, elliptic, 0.57-0.69 × 0.22-0.29 mm, slightly acute at apex, truncate at base; testa dark brown, reticulate.

Flowering \& fruiting: Flowering from July to early September and fruiting from late August to October.

Habitat: On moist slopes and in rock crevices, in association with Cyanotis obtusa Trim. (Commelinaceae), Christella sp. (Thelypteridaceae) and some grass species.

Distribution: Endemic to the southern Western Ghats (Fig. 17).

Specimens examined: INDIA, Kerala, Idukki district, Devikulam, Pallivasal, 01.07.1944, N.R. Yull 236 (CAL); Eravikulam National Park, 22.08.2014, A.P. Janeesha \& Santhosh Nampy 137612; Ibid., 22.08.2014, S. Syam Radh, Prashob \& A.P. Janeesha 134192; Ibid., 19.05.2015, A.P. Janeesha, S. Resmi \& V. Veena 137615; Eravikulam National Park, after view point, 19.05.2015, A.P. Janeesha \& Santhosh Nampy 137616 (CALI); near Idukki dam, 24.08.1981, V.S. Raju 71173 (MH); Pettimudi, 30.10.2012, Shaju \& Shareef, 74028; Rajamalai, 16.09. 1998, S.D. Biju 35857; Ibid., 27.10.1998, S.D. Віји 38393 (TBGT).

Conservation status: The species is currently known only from four localities in one district outside protected areas with an Extent of Occurrence (EOO) of $c .74 \mathrm{~km}^{2}$. The authors observed 58 plants of different growth stages, of which 41 were mature at two localities. The species is possibly surviving as fragmented populations in a narrow range of distribution, being subjected to habitat alteration/ destruction due to plantation activities and the growing tourism in those areas. It is therefore assessed here as Endangered (EN), B1ab(iii,iv,v), D (IUCN, 2019).

Notes: This is a robust plant with spatulate leaves and long scapes (about $50 \mathrm{~cm}$ long). The scapes, calyx and capsules are completely covered with golden brown woolly hairs.

Henckelia meeboldii (W.W.Sm. \& Ramaswami) A.Weber \& B.L.Burtt, Beitr. Biol. Pflanzen 70: 349. 1998 [“1997”]; T.S.Nayar et al., Fl Pl. Western Ghats 1: 528. 2014; Janeesha \& Nampy, Phytotaxa 268(1): 80. 2016. Didymocarpus meeboldii W.W.Sm. \& Ramaswami, Rec. Bot. Surv. India 6: 43. 1914; Gamble, Fl. Madras 2: 989. 1924; A.N.Henry et al., Fl. Tamil Nadu Ind., Ser I: Analysis 2: 132. 1987; M.Ahmedullah \& M.P.Nayar, Endemic Pl. Indian Region 1:143. 1987; Sasidh., Biodivers. Doc. Kerala Part 6: Fl. Pl. 332. 2004; T.S.Nayar et al., Fl. Pl. Kerala 342. 2006. Lectotype (designated by Janeesha \& Nampy, 2016): INDIA, Kerala, Peermade Ghat, s.d., Meebold 12851 (CAL [CAL0000019179!]; isolecto CAL [CAL0000019180!, CAL00000 19181!, CAL0000019182!], E [E00155177!], K [K000858198 digital image!).

Fig. 14

Perennials, rosette-forming hairy herbs with rootstocks. Roots adventitious, thin. Leaves 8-13, basal, decussate; petioles 4-12 cm long, pubescent, winged; wings $2-3 \mathrm{~mm}$ broad; laminae widely elliptic-ovate, $6-16 \times 5-11.5 \mathrm{~cm}$, obtuse to acute at apex, attenuate at base, margins crenate, sometimes slightly doubly crenate, ultimate segments regular, surfaces usually rugose, pubescent on both surfaces, but more so on the younger leaves and on lower veins, becoming less so with age; veins 5-7 on each side, much branched and conspicuous beneath. Cymes axillary, 1-5 per plant, 6-9 times divided, 10-18-flowered; peduncles terete, 10-26 cm long, reddish brown, glandular hairy; bracts two at each branching point, linear, 3-4 × 0.8-1 mm, acute at apex, margins entire, pilose; pedicels terete, 4-25 mm long, slender, glandular hairy. Flowers 13-15 × 11-13 mm. Calyx 5-partite, very deeply divided or basally connate; lobes ovate to lanceolate, unequal, posterior lobe small, 2-3.1 ×0.7-0.92 mm, others $3.2-5 \times 1-1.8 \mathrm{~mm}$, slightly acute at apex, margins entire, reddish brown, glandular hairy. Corolla zygomorphic, ventricose, two lipped, glandular hairy outside, glabrous inside; tube 9-11 $\times 3.7-4.2 \mathrm{~mm}$, dark lilac with a yellow patch in the 

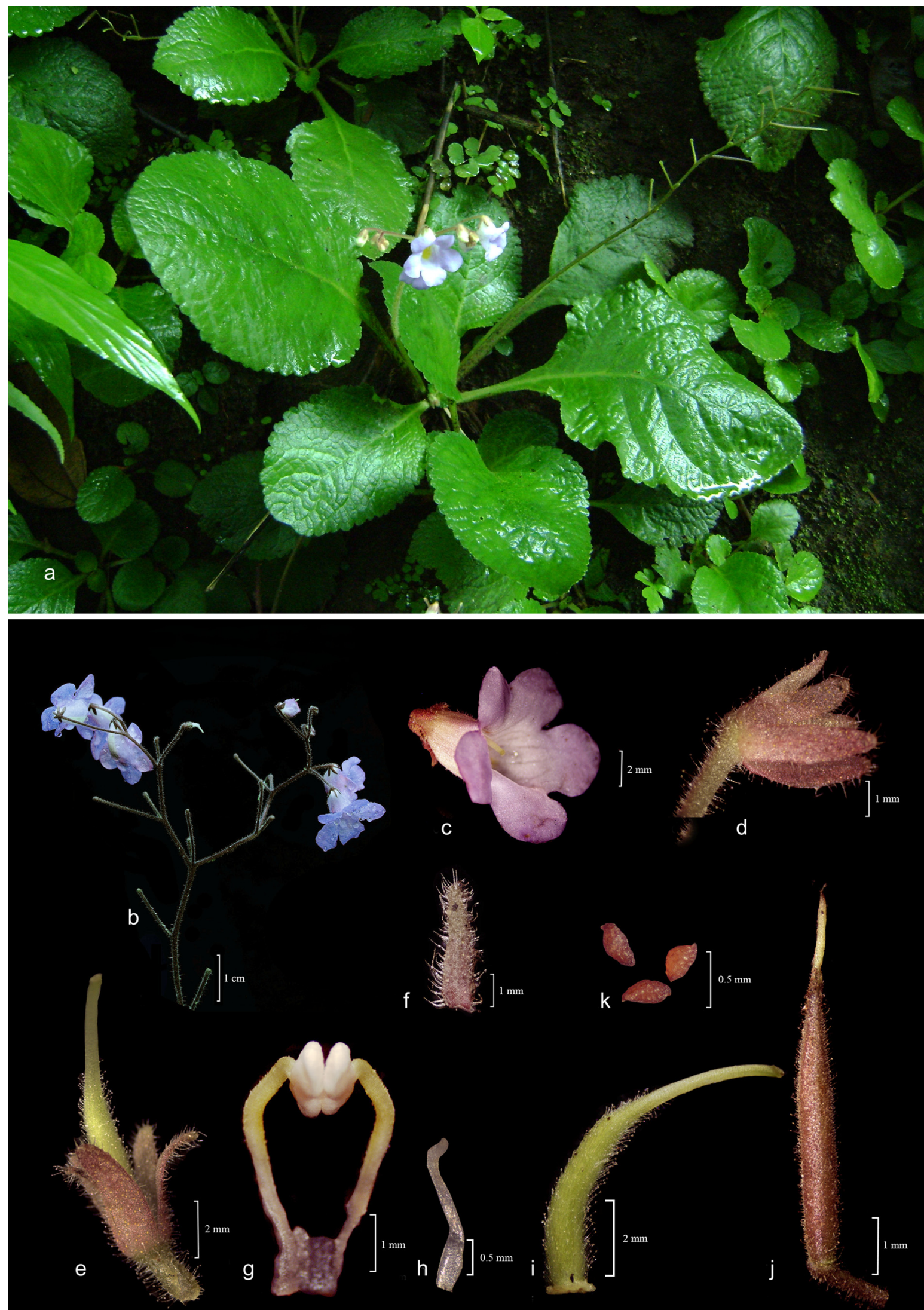

Fig. 14. Henckelia meeboldii (W.W.Sm. \& Ramaswami) A.Weber \& B.L.Burtt: a. Habit and habitat; b. A portion of the inflorescence; c. Flower; d. Calyx; e. Calyx with pistil; f. Bract; g. Stamens; h. Staminode; i. Pistil; j. Immature capsule; k. Seeds (from A.P. Janeesha \& K.M. Manudev 134202). 
throat; lobes ovate to slightly elliptic, rounded at apex, dark lilac, upper lip of two lobes, 3.7-4 ×5$5.8 \mathrm{~mm}$, lower lip of three lobes, 6-7 $\times 5-6 \mathrm{~mm}$. Stamens 2, adnate to the base of corolla, included; filaments 4-6 mm long, puberulent, green on the top and colourless towards base; anthers reniform, 1.5-1.7 × 0.9-0.95 mm, milky white, glabrous Staminodes 2; filaments $2.2-2.5 \mathrm{~mm}$ long, hyaline, glabrous; antherodes $0.42 \times 0.36 \mathrm{~mm}$, hyaline, glabrous. Ovary ovoid to oblong, 4.8-6.5 × 0.9$1.2 \mathrm{~mm}$, green, glandular hairy, sometimes slightly curved; style terete, 4-5.5 mm long, green, glabrous; stigma obliquely subcapitate, 0.3-0.35 × 0.2-0.25 mm, papillate, pale green. Capsules cylindrical, straight or slightly curved, 14-35 × 1.3$1.5 \mathrm{~mm}$, plagiocarpic, dehiscing loculicidally along the dorsal side, reddish brown, tomentulose with persistent stigma. Seeds numerous, elliptic, 0.38$0.45 \times 0.2-0.22 \mathrm{~mm}$, obtuse to slightly acute at apex, truncate at base; testa dark brown, reticulate.

Flowering \& fruiting: Flowering from July to October and fruiting from September to November.

Habitat: On moist slopes, in association with Adiantum philippenes L. (Pteridaceae), Christella sp. (Thelypteridaceae), Selaginella sp. (Selaginellaceae) and Cyanotis villosa Schult.f. (Commelinaceae).

Distribution: Endemic to the southern Western Ghats (Fig. 17).

Specimens examined: INDIA, Kerala, Ernakulam district, Shoolamudi, 11.07.2016, A.P. Janeesha, Manu Philip \& K. Shinoj 137692 (CALI); Idukki district, Mannavan shola, 24.05.2015, K.M. Manudev, S. Syam Radh \& A.P. Janeesha 137619; Munnar, 10.09.2013, A.P. Janeesha \& Santhosh Nampy 134214; near Kuttikanam-Kumali road, 30.06.2016, A.P. Janeesha \& P.S. Sreeja 137662; Neryamangalam to Adimali route, 25.07.2013, A.P. Janeesha \& K.M. Manudev 134202; OnnamthodeMathikettanshola National Park, 08.05.2015, S. Syam Radh \& A.P. Janeesha 137613 (CALI); Peerumedu, 31.08.1992, A. Nazarudeen 14386; Ibid., 12.09.2009, Geethakumary 58596 (TBGT); Pothumade-Munnar, 25.07.2013, A.P. Janeesha \&
K.M. Manudev 134203; Pynavu, 29.06.2016, P.S. Sreeja \& A.P. Janeesha 137667 (CALI); Upputhara, 30.09.1981, C.N. Mohanan 72086 (MH); Kottayam district, near Ottayitti, 26.06.2016, P.S. Sreeja \& A.P. Janeesha 137661; Vagamon churam, 07.08.2014, A.P. Janeesha \& A.P. Roshan 134273, 134274 , 134275; Ibid., 03.10.2015, C.Pramod \& A.P.Janeesha 137645; Vagamon churam-200 mtr after view point, 07.08.2014, A.P. Janeesha, A.P. Roshan \& Santhosh Nampy 134276 (CALI). Tamil Nadu, Madura district, Palani-Kodaikanal ghat road, 27.11.1985, K.M. Matthew \& N. Rajendran 43308; Tiger shola-acroos to Korappar, 01.08.1986, K.M. Matthew \& M. Charles 46094 (RHT).

Conservation status: This species is widely distributed in central areas of Kerala and some parts in western Tamil Nadu, with an Extent of Occurrence (EOO) of c. $3800 \mathrm{~km}^{2}$. More than 240 mature plants were observed from nine populations across Kerala. The estimated Area of Occupancy (AOO) for all localities is an estimated $48 \mathrm{~km}^{2}$. The majority of the localities are not in protected areas and are subjected to human disturbance due to plantation activities and tourism. Because if the small number of mature plants we consider the species to be of Endangered (EN) D, according to IUCN (2019) criteria.

Notes: Ramaswamy (1914) described the corolla tube and filaments as glabrous. But in the present investigation, the outside of the corolla tube was found to be glandular hairy and the filaments bearded. The species is morphologically close to $H$. humboldtiana, but can be distinguished by its broadly elliptic to ovate leaves, glandular indumentum on the pedicel, calyx lobes, corolla tube and ovary.

Henckelia missionis (Wall. ex R.Br.) A.Weber \& B.L.Burtt, Beitr. Biol. Pflanzen 70: 350. 1998[“1997”], nom. cons. prop;; T.S.Nayar et al., Fl. Pl. Western Ghats 1: 528. 2014. Didymocarpus missionis Wall. ex R.Br., Pl. Jav. Rar. 119. 1839; Rama Rao, Fl. Pl. Travancore 295. 1914; Gamble, Fl. Madras 2: 988. 1924; A.N.Henry \& Swamin., J. Bombay Nat. Hist. Soc. 76: 373. 1977; A.N.Henry 
et al., Fl. Tamil Nadu Ind., Ser I: Analysis 2: 132. 1987; M.Ahmedullah \& M.P.Nayar, Endemic Pl. Indian Region 1: 143. 1987; M.P.Nayar \& Sastry, Red Data Book Indian Pl. 3: 157. 1990; K.S.Walter \& H.J.Gillet, IUCN Red List Threatened Pl. 291. 1997; Gopalan \& A.N.Henry, Endemic Pl. India 114. 2000; T.S.Nayar et al., Fl. Pl. Kerala 342. 2006. Roettlera missionis (Wall. ex R.Br.) Kuntze, Revis. Gen. Pl. 2: 476. 1891. Type (cons. prop.): INDIA, Tamil Nadu, Kanyakumari district, KilaviarumalaiBalamore, 480 m, 28.07.1977, A.N. Henry 49421 (MH!).

Henckelia membranacea (Bedd.) Janeesha \& Nampy comb. nov. Didymocarpus membranacea Bedd., Icon. Pl. Ind. Or. 1: 39. 1874. Lectotype (designated here): Beddome's Icon Pl. Ind. Or. 1: t. 176. 1874. Figs. 15 \& 16 Perennials, hairy herbs with rootstocks. Roots adventitious, thin. Stems reduced. Leaves 6-10, basal, decussate; petioles 11-16 cm long, pubescent, not winged; laminae ovate to elliptic, 7.9-12.1 × $5.2-7.3 \mathrm{~cm}$, membranaceous, acute to acuminate at apex, obliquely cordate at base, margins entire, with white pellucid hairs, strigose on the upper surface, surfaces rough; veins usually 6 on each side, much branched and conspicuous beneath. Cymes axillary, 1-5 per plant, 3-5 times divided, 6-10flowered; bracts two, linear, $4 \mathrm{~mm}$ long; peduncles terete, 20-29 cm long, with scaly hairs towards apex; pedicels terete, 6-9 $\mathrm{mm}$ long, slender, pubescent. Flowers 20-25 mm long. Calyx 5partite, very deeply divided or basally connate; lobes linear to lanceolate, 5-8 mm long, acute at apex, margins entire, pilose. Corolla zygomorphic, subinfundibuliform, upper lip of two lobes, lower lip of three lobes, glabrous; tube pale lilac with a yellow patch in the throat; lobes pale lilac, ovate, rounded at apex. Stamens 2, adnate to the base of corolla, included, glabrous; anthers reniform, bearded. Staminodes 2; antherodes present. Ovary ovoid; style terete, glabrous; stigma obliquely subcapitate. Capsules cylindrical, 27-33 mm long, plagiocarpic, dehiscing loculicidally along the dorsal side, with persistent stigma. Seeds not seen.
Flowering \& fruiting: Flowering from May to July and fruiting from July to September.

Habitat: On moist slopes and in rock crevices.

Distribution: Endemic to the southern Western Ghats (Fig. 17).

Specimen examined: INDIA, Tamil Nadu, Kanyakumari district, Kalikesam river side, Balamore, 700 m, 31.08.1976, A.N. Henry 48151 (MH).

Conservation status: The population status at the type locality (A.N. Henry 49421) is presently uncertain, since no plants were found during recent fieldwork in 2014. The situation of the nearby population at Kalikesam river side at Balamore (A.N. Henry 48151) is presently unknown. Because of these uncertainties, the species should be regarded as Data Deficient (DD) according to IUCN guidelines (IUCN, 2019).

Typification: Henckelia missionis (Wall. ex R.Br.) A.Weber \& B.L.Burtt (Gesneriaceae) was first named (nom. nud.) as "Didymocarpus? missionis" by Wallich (1829). Subsequently Brown (1839) validated the preceding name with a description ("Caule brevissimo, pedunculis axillaribus scapiformibus, folis cordato ovatis") and as type, he referred to "Wall. List. no. 639". Kuntze (1891) transferred this species to Roettlera as R. missionis (Wall. ex R.Br.) Kuntze while Weber and Burtt (1998[“1997”]) placed this species in Henckelia as H. missionis (Wall. ex R.Br.) A.Weber \& B.L.Burtt. Meanwhile, Beddome (1874: 39, t. 176) described another species, viz., Didymocarpus membranaceus Bedd. from Pachemallai hills (Tamil Nadu) in South India as "leaves all radical, ... membranaceous, from obliquely cordate-ovate to cordate-lanceolate, corol-tube subinfundibuliform, ........ Clarke (1883) synonymised Beddome's species under D. missionis and this treatment was followed by subsequent authors (Clarke, 1885[“1884"]; Gamble, 1924; Henry et al., 1987). This species is endemic and very rare in South India (Nayar \& Sastry, 1990; Walter \& Gillet, 1997), and has been re-collected 


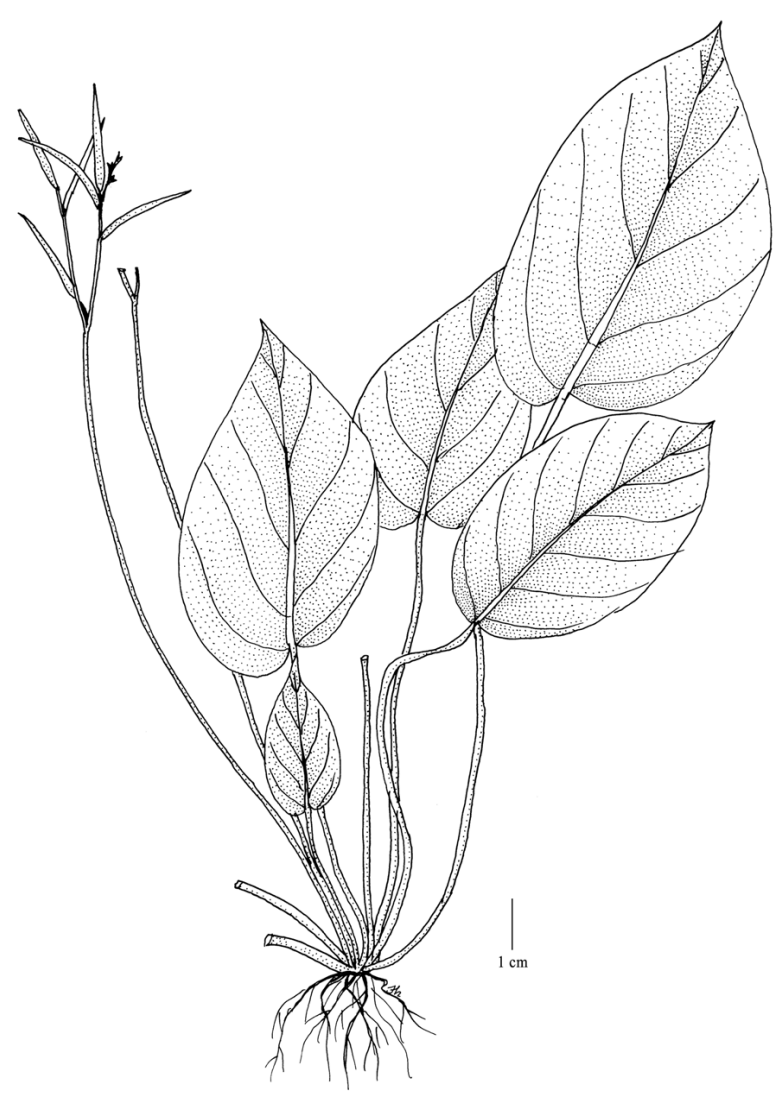

Fig. 15. Henckelia missionis (Wall. ex R.Br.) A.Weber \& B.L.Burtt (from A.N. Henry 49421, drawn by A.P. Janeesha).

only twice from Kanyakumari district in Tamil Nadu (Madras Herbarium: Henry 48151, 49421).

While examining "Wallich List. No. 639" (as cited by Brown l.c.), it was found that Brown's (l.c.) citation refers to Arenaria globiflora Wallich (1829: 19), belonging to Caryophyllaceae. It was found that Wallich's Didymocarpus missionis is more likely referable to "Wall. List. No. 6396" (probably a typographical error by Brown). According to Stafleu and Cowan (1988), Wallich's main collections are kept at $\mathrm{K}(\mathrm{K}-\mathrm{W})$. After a thorough search in various herbaria, two sheets of "Wall. No. 6396" were found, one each at BM and K. The lone sheet in BM (BM000617800 digital image!) has two flower buds separately mounted and carries two labels with annotations. The top one, probably the original label reads "Didymocarpus missionis Wall. Cat 6396 Herb Madras" and the second one, made by curators at BM "Wallich 6396: TYPE

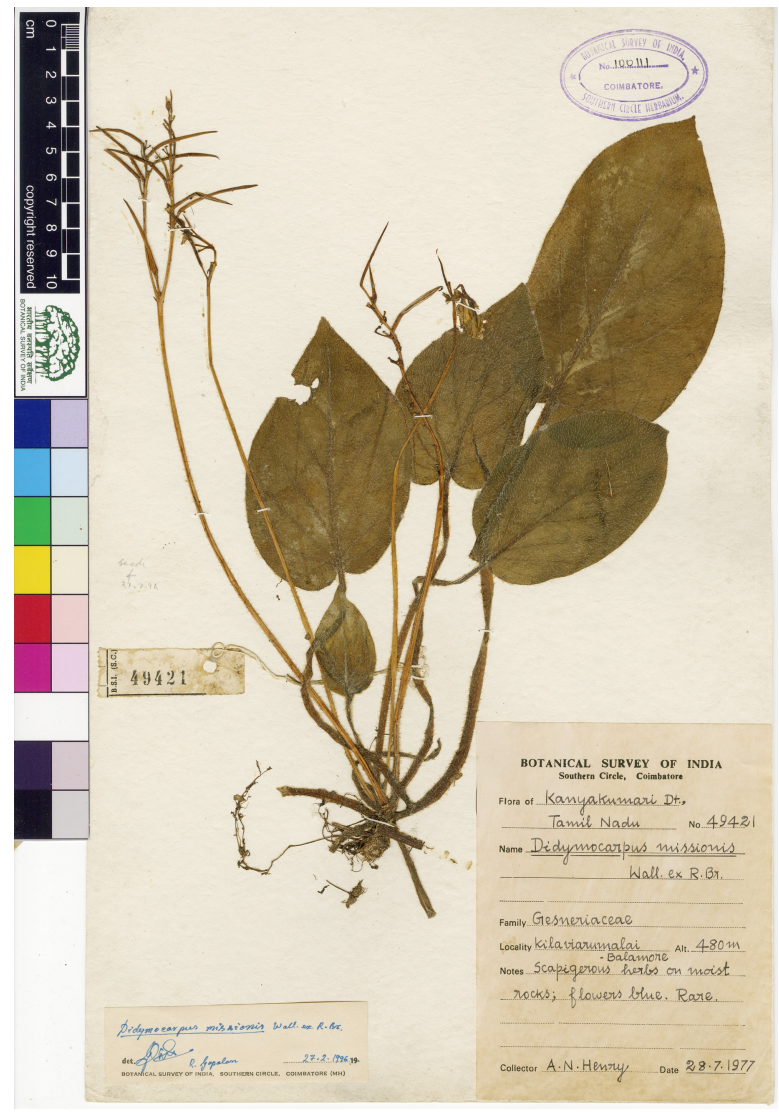

Fig. 16. Type (conserved) of Henckelia missionis (Wall. ex R.Br.) A.Weber \& B.L.Burtt. @ Botanical Survey of India, Kolkata, Reproduced with permission.

SPECIMEN Didymocarpus Wall. ex R.Br., Cyrtandreae: 119 (1839)" respectively. The flower buds are not, however, unquestionably referable to H. missionis. The sheet at K (K001123777 digital image!) has a single specimen with three scapes and a few leaves. There is an inscription on the sheet, possibly by C.B. Clarke written in pencil: 'Didymocarpus missionis Wall. C.B.Clarke (= D. membranaceus Beddome Ic. t. 176)'. This sheet also carries a Wallich label on which is written: "6396 Didymocarpus? missionis Wall (caet. spec. 777-793). Hb. Madras". However, the ovate leaves with cordate base, long pedicels, infundibuliform corolla, and persistent calyx undoubtedly indicate that the specimen belongs to Jerdonia indica Wight (Wight, 1848: 10, t. 1352) (Gesneriaceae). The original description by Brown (1839) is applicable equally to $J$. indica also. Since the type specimens of $H$. missionis is demonstrably ambiguous and doesn't 


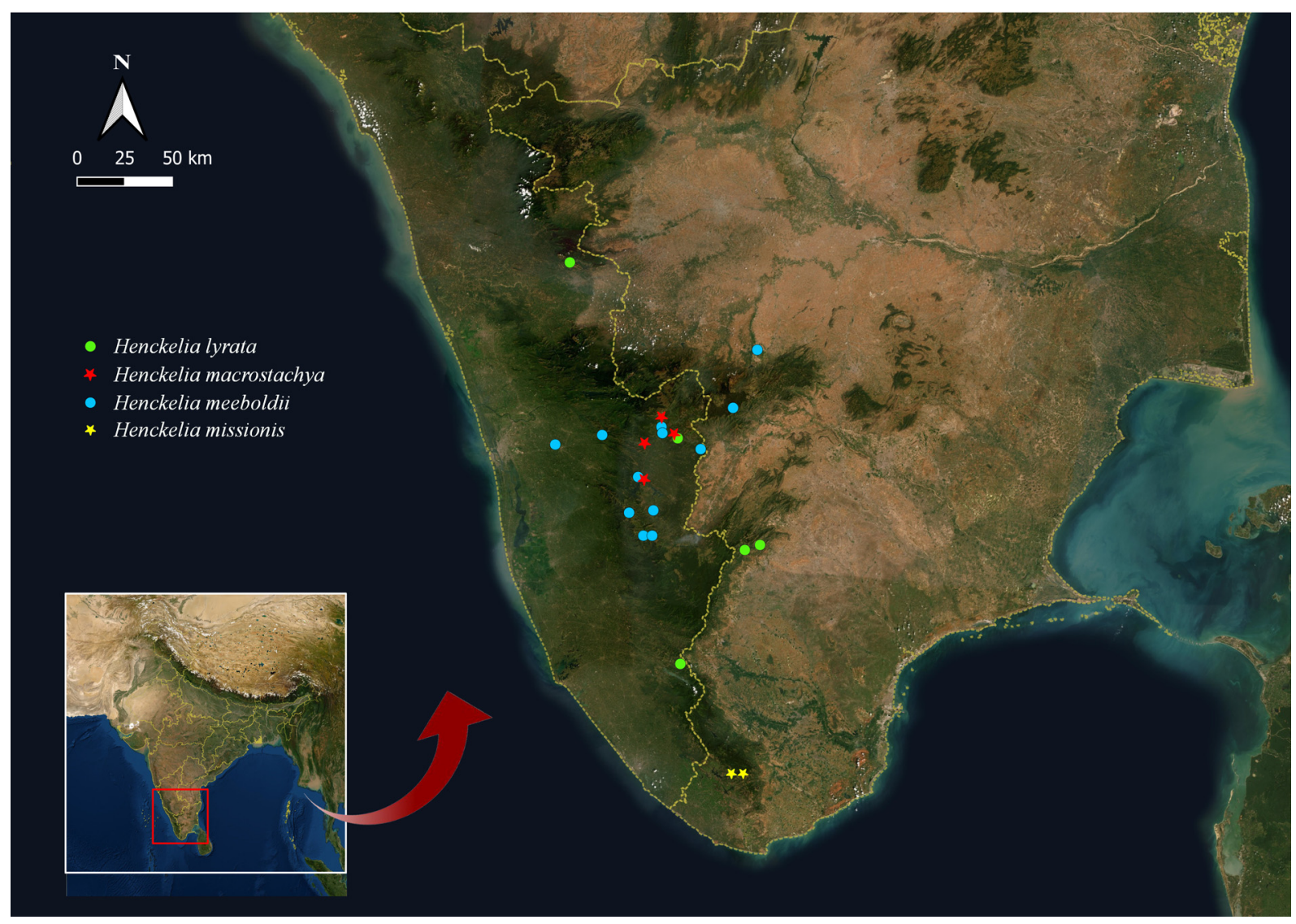

Fig. 17. Distribution of Henckelia lyrata (Wight) A.Weber \& B.L.Burtt, H. macrostachya (E.Barnes) A.Weber \& B.L.Burtt, H. meeboldii (W.W.Sm. \& Ramaswami) A.Weber \& B.L.Burtt and H. missionis (Wall. ex R.Br.) A.Weber \& B.L.Burtt in South India (drawn using QGIS v.3.12.1).

permit a precise identification and application of the name, the name is illegitimate (Art. 52.1) and a proposal is under preparation to conserve the name with a conserved type according to Articles 14.1 and 14.9 of ICN (Turland et al., 2018).

Beddome (1874) described Didymocarpus membranacea Bedd. based on his specimens from "Pachemallay (2000 feet elevation), in South Travancore ghats", India. According to TL-2 (Stafleu \& Cowan, 1976), the herbarium and type materials of R.H. Beddome are mainly at BM, some perhaps at $\mathrm{K}$ and CAL. Even after extensive search no relevant materials have been found in these herbaria. Hence the illustration in the protologue (Bedd., Icon Pl. Ind. Or. 1: t. 176. 1874) is selected here as the lectotype, according to articles 9.3 and 9.4 of ICN (Turland et al., 2018).

Henckelia ovalifolia (Wight) A.Weber \& B.L.Burtt, Beitr. Biol. Pflanzen 70: 351. 1998[“1997”]; T.S.Nayar et al., Fl. Pl. Western
Ghats 1: 529. 2014; Janeesha \& Nampy, Phytotaxa 268(1): 84. 2016. Didymocarpus ovalifolius Wight, Icon. Pl. Ind. Orient. 4: 24. t. 1351. 1848, Ill. Ind. Bot. 2: 131. t. 142, fig. 6. 1850; C.B.Clarke in Hook.f., Fl. Brit. India 4: 354. 1885[“1884”]; Rama Rao, Fl. Pl. Travancore 295. 1914; Gamble, Fl. Madras 2: 988. 1924; M. Ahmedullah \& M.P.Nayar, Endemic Pl. Indian Region 1: 143. 1987; A.N.Henry et al., Fl. Tamil Nadu Ind., Ser I: Analysis 2: 132. 1987; Manilal, Fl. Silent Valley 200. 1988; M.Mohanan \& A.N.Henry, Fl. Thiruvananthapuram Dist. 338. 1994; Gopalan \& A.N.Henry, Endemic Pl. India 117. 2000; M.Mohanan \& Sivad., Fl. Agasthyamala 495. 2002; Sasidh., Biodivers. Doc. Kerala part 6: Fl. Pl. 332. 2004; T.S. Nayar et al., Fl. Pl. Kerala 342. 2006. Roettlera ovalifolia (Wight) Kuntze, Revis. Gen. Pl. 2: 476. 1891. Lectotype (designated by Janeesha \& Nampy, 2016): INDIA, Tamil Nadu, Courtallam, 08.1835, Wight 558 (E [E00155178 digital image!]; 
isolecto E [E00179262 digital image!], K [K000858199 digital image!]).

Fig. 18

Perennials, rosette-forming herbs with rootstock. Roots adventitious. Stem reduced. Leaves 8-18, decussate; petioles 4-14 cm long, hairy throughout, not winged; laminae usually ovate-broadly ovate, rarely orbicular, $3.3-12.8 \times 2.5-7.7 \mathrm{~cm}$, slightly acute to obtuse at apex, unequal at base, margins crenate to serrate, white strigose or tomentose above and on the lower veins; veins 7-10 on each side, much branched and conspicuous beneath. Cymes axillary, 2-5 per plant, 2-3 times divided, 6-8flowered; bracts two, oblanceolate, $6.38 \times 1.34 \mathrm{~mm}$, white tomentose hairy out; peduncles terete, 9-22.5 $\mathrm{cm}$ long, green-pale reddish brown, hairy throughout; pedicels terete, 5-10 mm long, narrow, hairy. Flowers 12-20 × 8-15 mm. Calyx 5-partite, very deeply divided or basally connate; lobes linear to lanceolate, unequal, posterior lobe small, 4.2$5.1 \times 0.8-1 \mathrm{~mm}$, others 5.8-6.8 × 1.1-1.2 mm, slightly acute at apex, margins entire, reddish brown, white tomentose outside. Corolla zygomorphic, ventricose, two lipped, white hirsute outside, glabrous inside; tube 7.2-11.3 × 5-5.8 mm, very pale violet to brilliant purple, deep purple at the throat and a yellow blotch at the base of the tube; lobes very widely ovate, slightly acute to obtuse at apex, violet to brilliant purple, upper lip of two lobes, $6.5-7.2 \times 5.8-6.6 \mathrm{~mm}$, lower lip of three lobes, $8-8.4 \times 7.1-7.25 \mathrm{~mm}$. Stamens 2 , adnate to the base of the corolla, included; filaments 5.3$5.7 \mathrm{~mm}$ long, puberulent towards the apex, light yellowish green towards the top and milky white towards the base; anthers reniform, 1.7-1.9 × 0.65$0.8 \mathrm{~mm}$, yellowish brown, glabrous. Staminodes 2; filaments $3.5-3.7 \mathrm{~mm}$ long, hyaline, glabrous; antherodes not prominent. Ovary narrowly ovoid to cylindrical, tapering to a style, 4.9-5 × 1.1-1.21 $\mathrm{mm}$, green towards the base and reddish brown towards the apex, hairy; style terete, 4-4.1 × 0.35$0.40 \mathrm{~mm}$ long, hyaline, hairy throughout up to the middle; stigma obliquely subcapitate, $0.68 \times 0.38$ $\mathrm{mm}$, papillate, milky white. Capsules linear, straight, 36-44 × 2-2.2 mm, plagiocarpic, dehiscing loculicidally along the dorsal side, reddish brown, hairy with persistent stigma and calyx. Seeds numerous, elliptic, 0.49-0.59 × 0.21-0.24 mm, acute at apex, truncate at base; testa dark brown, reticulate.

Flowering \& fruiting: Flowering from June to September and fruiting from August to October (up to February).

Habitat: In moist shady areas of dense evergreen forest, in humus rich soil or on fallen, moss covered tree trunks. Grows in association with Impatiens travancorica Bedd. (Balsaminaceae), Christella sp. (Thelypteridaceae), Osbeckia sp. (Melastomataceae) and some grass species.

Distribution: Endemic to the southern Western Ghats (Fig. 17).

Specimens examined: INDIA, Kerala, Idukki district, Kallar Reserve Forest, 16.07.1989, s.coll. 90687 (MH); Periyar-Koruthode, 25.09.1996, Jomy Augustine 16811 (CALI); Kollam district, Thenmala, 15.06.1984, N. Mohanan 165 (TBGT); Palakkad district, Nillikkal South, 08.06.1982, Prasannan 10392 (CALI); Thiruvananthapuram district, Agasthyamala, 24.06.1993, N. Mohanan 11454 (CALI, TBGT); Ibid., 19.10.1993, G. Gangaprasad \& S. William Decruse 18419 (TBGT); Ibid., 06.06.2014, K.M. Manudev \& A.P. Janeesha 138919, 138925; Ibid., 21.10.2016, K. Shinoj \& A.P. Janeesha 137658 (CALI); Pongalapara, 25.08.1990, N. Mohanan 10044 (TBGT); Ponmudi, 10.10.2014, K.P. Vimal, M.G. Prasad \& A.P. Janeesha 137602 (CALI); Western slopes of Agasthyamala, 06.10.1973, J. Joseph 44608 (MH); Wayanad district, Gurukula botanical sanctuary (cultivated), 25.11.2014, A.P.Janeesha \& S. Resmi 137609; Periya, 25.08.1984, R.T. Balakrishnan 40450; Thalapaya, 10.02.1986, R.T. Balakrishnan 41944 (CALI). Tamil Nadu, Kanyakumari district, Muthukuzhivayal, 06.08.1977, A.N. Henry 49610 (MH); Ibid., 01.09.1981, K.M. Matthew 17788 (RHT); Tirunelveli district, Agasthyamala, 22.06.1901, C.A. Barber 2918; s.loc., 1807, R.H. Beddome 164 (MH). 

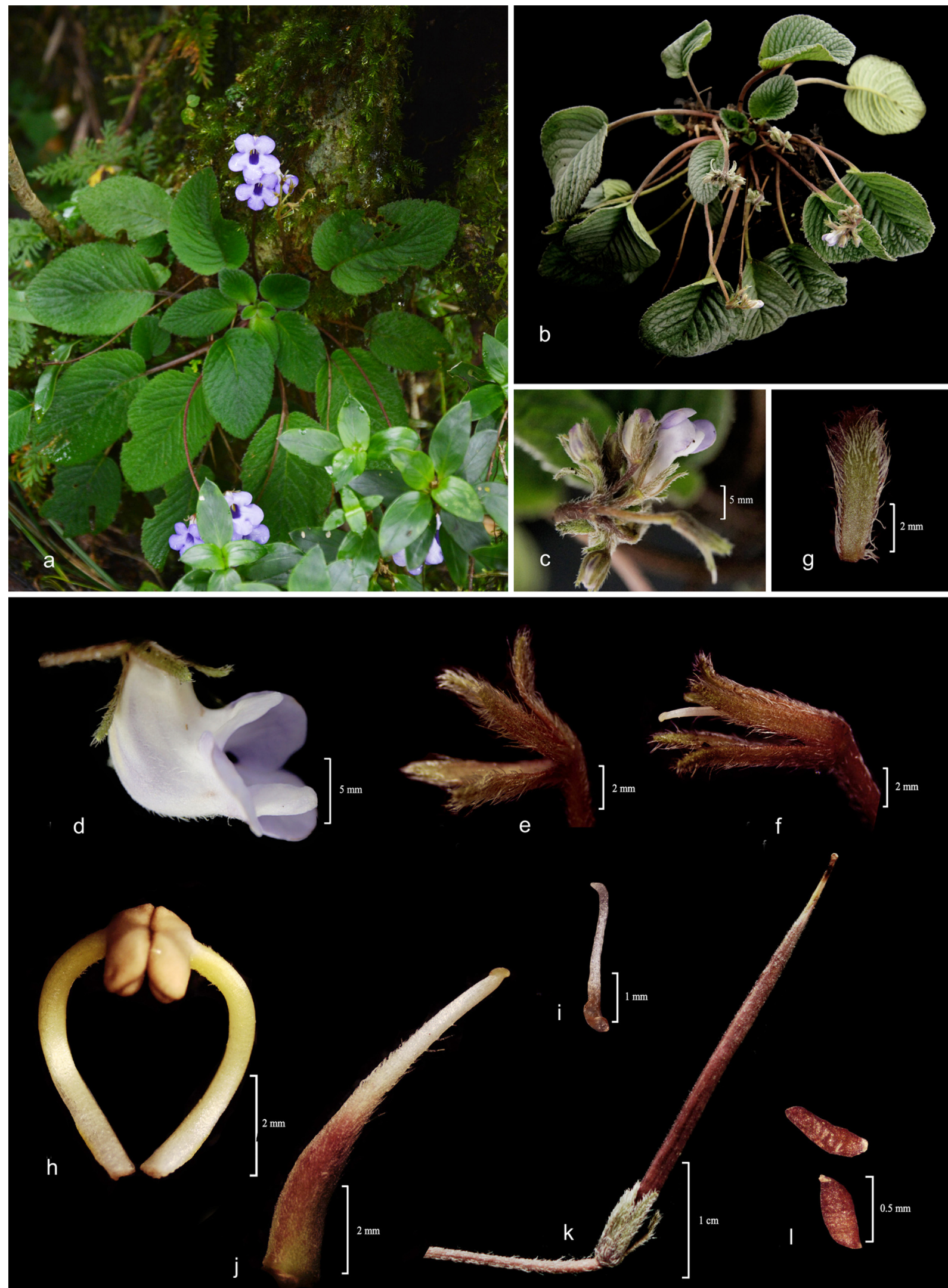

Fig. 18. Henckelia ovalifolia (Wight) A.Weber \& B.L.Burtt: a. Plant in natural habitat; b. Habit; c. A portion of the inflorescence; d. Flower; e. Calyx; f. Calyx with pistil; g. Bract; h. Stamens; i. Staminode; j. Pistil; k. Immature capsule; I. Seeds (a from K.P Vimal, M. G. Prasad \& A.P. Janeesha 137602; b-I from K.M. Manudev \& A.P. Janeesha 138919). 
Conservation status: This species is known from eight locations in seven districts in Kerala and Tamil Nadu with a narrow South-North distribution. The estimated Extent of Occurrence (EOO) is c. 1100 $\mathrm{km}^{2}$ and Area of Occupancy (AOO) is $32 \mathrm{~km}^{2}$. The authors surveyed three locations and found a total of only 81 mature plants. Since these locations are inside protected areas, the chances for a decline due to anthropogenic activity is small. The situation of just under half of the localities is known. However, when extrapolating the findings on mature plants to the entire distribution range, it may justify categorisation of the species as Endangered (EN), D (IUCN, 2019).

Notes: Henckelia ovalifolia and H. missionis are similar in having simple ovate, long petioled leaves, large flowers and long fruits. However, $H$. ovalifolia can be identified by its leaves with serrate margins and strigose hairs, scapes hairy throughout, hirsute corolla, puberulent filaments and a hairy style.

Henckelia pradeepiana Nampy, Manudev \& A.Weber., Rheedea 22(2): 119. 2012; T.S.Nayar et al., Fl. Pl. Western Ghats 1: 529. 2014. Type: INDIA, Kerala, Kozhikode district, Muthappanpuzha, N $11^{\circ} 26.6992 '$, E 07605.2882', 470 m, 19.08.2010, Santhosh Nampy \& K.M. Manudev 3102 (holo CALI!; iso DEV!, W!).

Fig. 19

Perennating tuberous herbs. Tubers $1-2.5 \mathrm{~cm}$ diam., discoid. Leaves usually 1 , rarely up to 3 ; petioles $1.8-5.6 \mathrm{~cm}$ long, sparsely glandular hairy, not winged; laminae broadly ovate or elliptic, oblong-ovate, 9-16 × 5.5-9 cm, acute at apex, slightly oblique or cordate at base, margins entire to serrulate, glabrous above, glandular hairy below, membranaceous, glandular hairy on the margins, surfaces smooth; veins usually 7 on each side, much branched and conspicuous beneath. Cymes axillary, 1-3 per plant, 1-6 times divided, 2-19-flowered, glandular hairy; peduncles terete, 5-20 cm long, green; bracts two at each branching point, lanceolate to triangular, 4.8-6.1 × 1.2-1.7 mm, acute at apex, margin entire, slightly hairy; pedicels terete, 1-3.8 cm long, slender, glandular hairy.
Flowers c. $10 \times 7 \mathrm{~mm}$. Calyx 5-partite, basally connate; lobes ovate-lanceolate, unequal, posterior lobe small, 3-4.1 × 1-1.25 mm, others 4.3-7 × 1.3$2.1 \mathrm{~mm}$, keeled, blunt at apex, margins entire, green, glabrous. Corolla campanulate, two lipped, glabrous inside and outside (but with a few hairs at the corolla mouth); tube 4-5.4 × 5.2-5.6 mm, lilac to pale lilac with a yellow patch in the throat, with 7-10 purplish streaks; lobes ovate to rounded at apex, lilac to pale lilac, upper lip of two lobes, 2$3.1 \times 3.4-4.51 \mathrm{~mm}$, lower lip of three lobes, 3-3.8 $\times 3.6-4.3 \mathrm{~mm}$. Stamens 2 , adnate to the base of corolla, included; filaments $2.4-3.3 \mathrm{~mm}$ long, glabrous, greenish yellow; anthers reniform, 1.3$1.5 \times 0.53-0.73 \mathrm{~mm}$, milky white, glabrous Staminodes 2; filaments 1-1.2 mm long, hyaline, glabrous; antherodes ovate to oblate, c. $0.31 \times 0.3$ mm. Ovary ovoid, 2-2.4 × 1.35-1.75 mm, green, glabrous; style terete, 4-5.22 mm long, green, glabrous; stigma subpeltate, lower lip strongly expanded and slightly emarginate at apex, 1.7-2.1 $\times 0.5-0.87 \mathrm{~mm}$, papillate, yellow to yellowish green Capsules ovoid to subglobose, 5.6-6.3 × 5-6 mm, plagiocarpic, dehiscing by a longitudinal slit on the upper side, greenish brown, with persistent calyx and a prominent beak. Seeds numerous, elliptic, $0.4-0.52 \times 0.15-0.2 \mathrm{~mm}$, acute at both ends; testa dark brown, reticulate.

Flowering \& fruiting: Flowering from July to August and fruiting from August to September.

Habitat: On moist rocks and mountain slopes, found in association with mosses and ferns.

Distribution: Endemic to the southern Western Ghats (Fig. 20).

Specimens examined: INDIA, Kerala, Kozhikode district, Muthappanpuzha, N $11^{\circ} 26.6992^{\prime}$, E $76^{\circ} 05.2882 ', 470$ m, 18.07.2010, K.M. Manudev 3022 (DEV); Ibid., 19.08.2010, A.K. Pradeep 90089 (CALI); Ibid., 19.08.2010, K.M. Manudev \& Santhosh Nampy 3105; Ibid., 17.09.2011, Santhosh Nampy \& K.M. Manudev 4547 (DEV); Ibid., 12.08.2014, A.P. Janeesha \& Santhosh Nampy 134287, 134288; Ibid., 15.06.2015, A.P. Janeesha \& S. Resmi 137624; Muthappanpuzha-Kundanthode, 15.06.2015, A.P. 


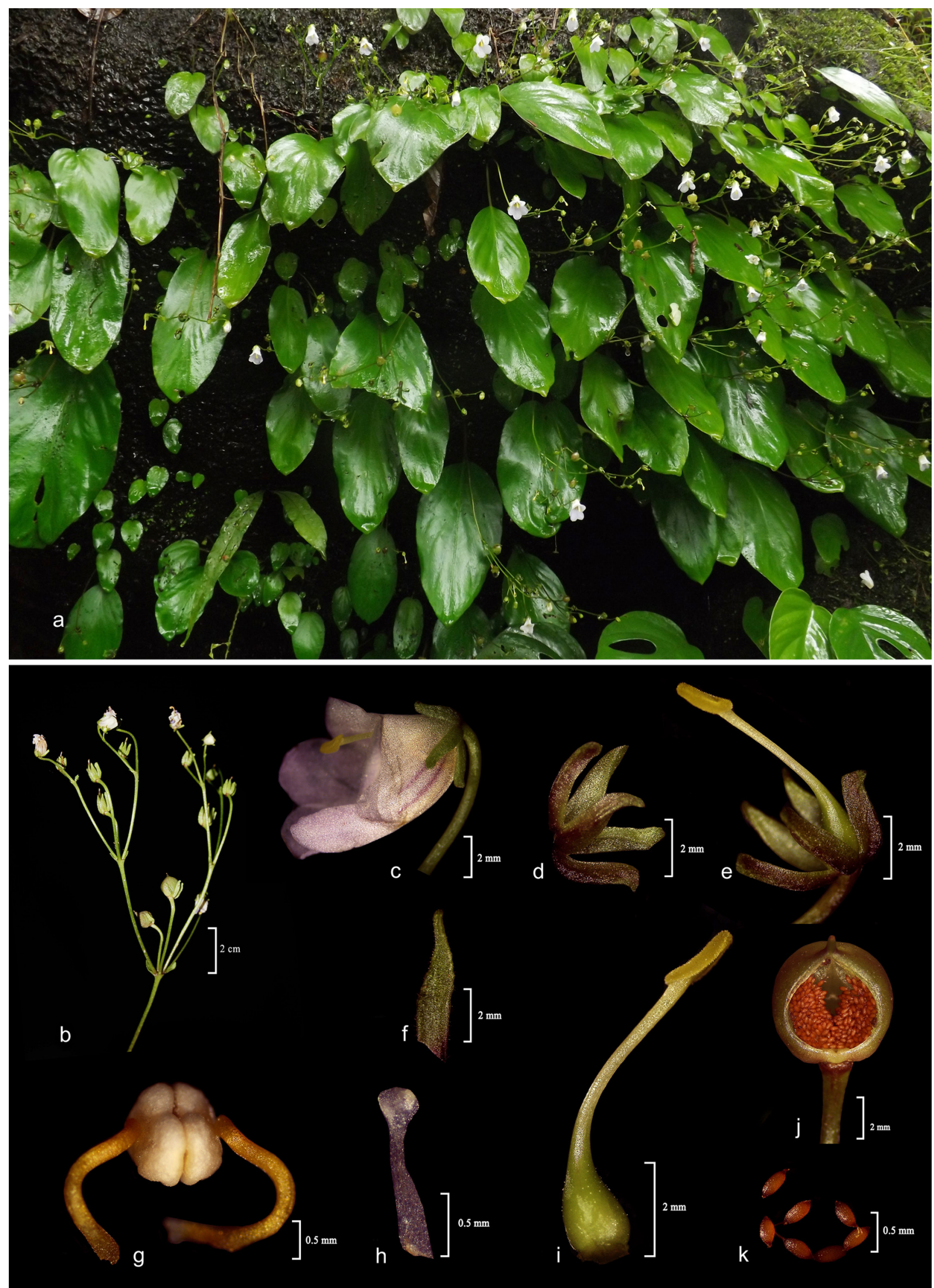

Fig. 19. Henckelia pradeepiana Nampy, Manudev \& A.Weber: a. Habit and habitat; b. A portion of the inflorescence; c. Flower; d. Calyx; e. Calyx with pistil; f. Bract; g. Stamens; h. Staminode; i. Pistil; j. Dehiscing capsule; k. Seeds (from A.P. Janeesha \& Santhosh Nampy 134287). 


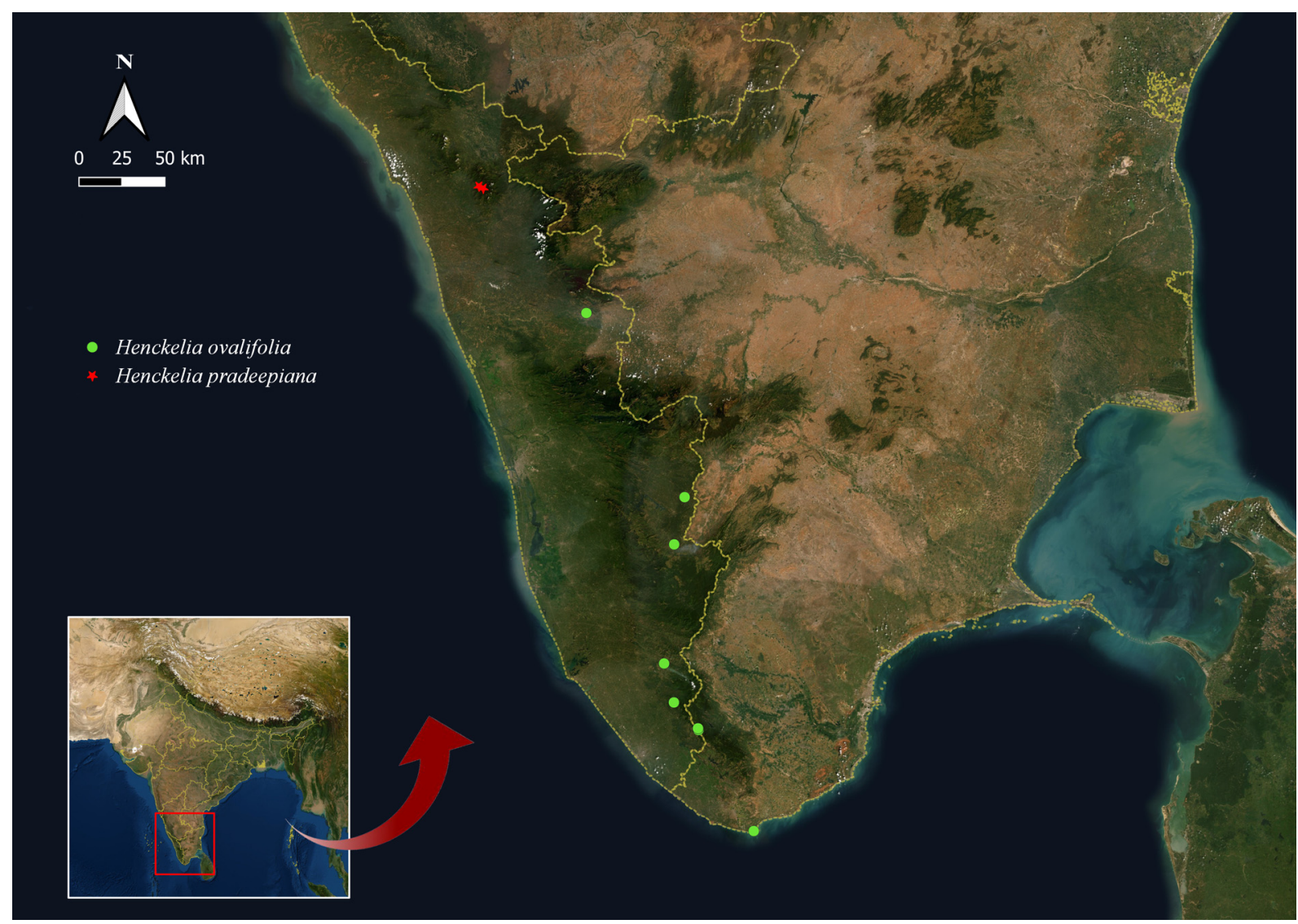

Fig. 20. Distribution of Henckelia ovalifolia (Wight) A.Weber \& B.L.Burtt and H. pradeepiana Nampy, Manudev \& A.Weber in South India (drawn using QGIS v.3.12.1).

Janeesha \& S. Resmi 137623; way to VellarimalaOlichuchattam, N $11^{\circ} 25.7842^{\prime}$, E $76^{\circ} 05.1732^{\prime}$, 1160 m, 19.09.1997, A.K. Pradeep 56009 (CALI).

Conservation status: The species is currently known only from the type locality which is an unprotected area, with two subpopulations separated by a distance of 2 kilometres. The locality has a total of 79 mature plants, with the possibility of future disturbances due to human activities since the area is near a stream which is used by local people for their daily needs. The Area of Occupancy (AOO) is less than $10 \mathrm{~km}^{2}$. The species may be categorized as Critically endangered (CR), B2ab(ii,iii,iv,v), D (IUCN, 2019).

Notes: This species has several unusual features not found in any other Henckelia species in South India or the genus as a whole. This is the only species of Henckelia having tubers to enable the plants to overcome unfavourable conditions or periods. The plants usually have only one leaf, but sometimes up to 3 arranged alternately. The stigma is unusual for South Indian Henckelia in that they are chiritoid, with the upper lobe reduced and the lower one undivided and linguate (Weber et al., 2011; Manudev et al., 2012). The capsules are globose in contrast to the linear to cylindrical ones in other South Indian Henckelia species or any other species in the genus, but are plagiocarpic and dehisce similar to other South Indian Henckelia. They are reminiscent of those found in species of Loxocarpus, another genus previously included in Henckelia (Weber \& Burtt, 1998[“1997”]) but recently resurrected (Weber et al., 2011; Middleton et al., 2013).

Henckelia repens (Bedd.) A.Weber \& B.L.Burtt, Beitr. Biol. Pflanzen 70: 354. 1998[“1997”]; T.S.Nayar et al., Fl. Pl. Western Ghats 1: 529. 2014. Didymocarpus repens Bedd., Icon. Pl. Ind. Or. 1: 24. t. 120. 1874; C.B.Clarke in Hook.f., Fl. Brit. India 4: 354. 1885[“1884”]; Rama Rao, Fl. Pl. Travancore 
295. 1914; Gamble, Fl. Madras 2: 987. 1924; M.Ahmedullah \& M.P.Nayar, Endemic Pl. Indian Region 1: 143. 1987; A.N.Henry et al., Fl. Tamil Nadu Ind., Ser I: Analysis 2: 133. 1987; M.Mohanan \& A.N.Henry, Fl. Thiruvananthapuram Dist. 338. 1994; Gopalan \& A.N.Henry, Endemic Pl. India 121. 2000; M.Mohanan \& Sivad., Fl. Agasthyamala 496. 2002; Sasidh., Biodivers. Doc. Kerala Part 6: Fl. Pl. 332. 2004; T.S.Nayar et al., Fl. Pl. Kerala 342. 2006. Rottlera repens (Bedd.) Kuntze, Revis. Gen. Pl. 2: 477. 1891. Lectotype (designated by Vitek et al., 2000): INDIA, Tinnevelly and Travancore hills, 3000 feet elevation, 09.1868, Beddome 46 (K [K000858200 digital image!]; isolecto $\mathrm{MH}$ [36684!, $36680 !])$.

Fig. 21

Perennial creeping herbs, rooting at nodes and also along the internodes, hairy throughout. Roots adventitious. Stems slender, terete, hairy; internodes $6-12 \mathrm{~cm}$ long. Leaves two per node, one is highly reduced to a scale; petioles $3.5-18 \mathrm{~cm}$ long, not winged; laminae broadly ovate to sub-orbicular, $5.2-10 \times 5-8.3 \mathrm{~cm}$, acute at apex, cordate at base, margins doubly crenate, hairy; veins usually 5-6 on each side, much branched and conspicuous beneath. Cymes few-flowered, usually one from each node, 1-3 times divided, 2-4-flowered; peduncles 4-14.5 cm long, terete, green-pale reddish brown, hairy throughout; pedicels 5-10 $\mathrm{mm}$ long, terete, narrow, glandular hairy. Flowers 12-20 × 8-15 mm. Calyx 5-partite, very deeply divided or basally connate; lobes broadly lanceolate, slightly acute at apex, reddish brown, margins entire, hairy, unequal, posterior one small, 3.4-3.7 $\times 0.7-0.83 \mathrm{~mm}$, others $3.8-4.2 \times 0.83-1 \mathrm{~mm}$. Corolla zygomorphic, ventricose, two lipped, glandular hairy outside, glabrous inside; tube 12$13 \times 4.5-5.4 \mathrm{~mm}$, dark to pale lilac with a yellow blotch at the throat; lobes elliptic to oblong, rounded at apex, dark to pale lilac, upper lip of two lobes, 4.1-4.9 × 4.7-5.2 mm, lower lip of three lobes, 5-6.65 × 4.3-5.47 mm. Stamens 2, adnate to the base of the corolla, included; filaments 7.5-8 $\mathrm{mm}$ long, broad and hairy at the middle, not geniculate, yellow at the middle, other parts milky white; anthers reniform, 2-2.1 $\times 0.5-0.7 \mathrm{~mm}$, purple coloured except the cohering region of the lobes and also the region surrounding the connective. Staminodes 2; filaments $3.6-4.1 \mathrm{~mm}$ long, hyaline, hairy towards the middle; antherodes trilobed, $0.42-0.44 \times 0.36-0.37 \mathrm{~mm}$, prominent. Ovary narrowly ovoid, tapering to a style, c. $7.2 \times$ $1.21 \mathrm{~mm}$, green towards the base and reddish green towards the distal part, eglandular hairy; style terete, 4-4.4 × $0.5 \mathrm{~mm}$ long, hyaline, eglandular hairy throughout; stigma oblique, subpeltate, $1.7 \times 1.23$ $\mathrm{mm}$, glabrous. Capsules linear, straight, 20-22 $\times$ 2-2.2 mm, plagiocarpic, dehiscing loculicidally along the dorsal side, reddish brown, hairy, with persistent stigma. Seeds numerous, elliptic, 0.49$0.59 \times 0.21-0.24 \mathrm{~mm}$, slightly acute at apex, truncate at base; testa dark brown, reticulate.

Flowering \& fruiting: Flowering from (May) June to October and fruiting from August to December.

Habitat: Henckelia repens is found as an undercover in moist mixed evergreen and deciduous forests, in association with Elatostema sp., Pouzolzia sp. (both Urticaceae), Adiantum philippenes L. (Pteridaceae), Christella sp. (Thelypteridaceae), Selaginella sp. (Selaginellaceae) and some grass species.

Distribution: Endemic to the southern Western Ghats (Fig. 26).

Specimens examined: INDIA, Kerala, Kollam district, Kolatoor, s.d., s.coll. 1293 (MH); Thiruvananthapuram district, AgasthyamalaMutththatti, 06.06.2014, K.M. Manudev, A.P. Janeesha \& Santhosh Nampy 138940 (CALI); Athirumala, 12.08.1988, N. Mohanan 4227 (TBGT, CALI); Ibid., 07.06.2014, K.M. Manudev, P.G. Arun Kumar \& A.P. Janeesha 138936; Attayar, 13.05.1988, N. Mohanan 9614; Bonaccord, 27.04.1993, A. Nazarudeen 17128 (CALI); Ibid., 21.12.1987, N. Mohanan 9066 (TBGT, CALI); forest near Bonaccord, 21.08.1975, J. Joseph 46470; Ibid., 02.10.1973, J. Joseph 44493 (MH); Chemungi, s.d., Rajkumar 11775; Ibid., 18.08.1992, E.S. Santhosh Kumar \& M. Abdul Jabbar 14447; Ibid., 11.08.2011, P.M. Sheeba 66275 (TBGT); Pandimottai, 19.05.1979, M. Mohanan 61853 (MH); Pidichathamparamal, 31.05.2001, Non Wood Forest 


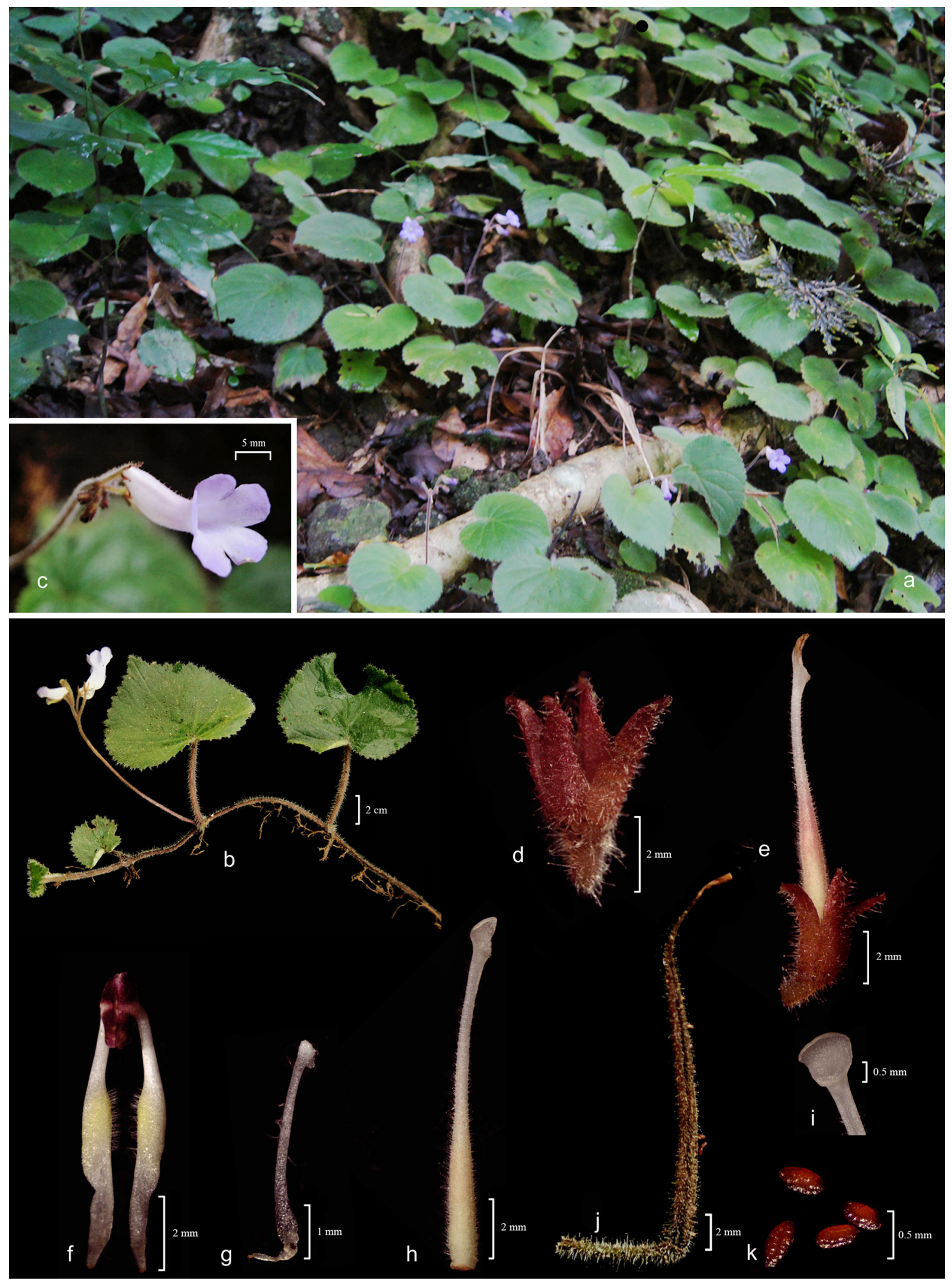

Fig. 21. Henckelia repens (Bedd.) A.Weber \& B.L.Burtt: a. Habit and habitat; b. Flowering creeping shoot; c. Flower; d. Calyx; e. Calyx with pistil; f. Stamens; g. Staminode; h. Pistil; i. Stigma; j. Capsule; k. Seeds (a \& b from K.M. Manudev, A.P. Janeesha \& Santhosh Nampy 138940 ; K.M. Manudev, P.G. Arunkumar \& A.P. Janeesha 138936). 
Product's (NWFPs) Team 46274; Pinarummodu, 25.09.2008, C.G. Vishnu 64727 (TBGT); Ponmudi, 28.11.2012, A.P. Janeesha, P.M. Shahina \& K.M. Manudev 134240; Ibid., 10.10.2014, M.G. Vimal, Prasad \& A.P. Janeesha 137603; s.loc., 21.12.1987, N. Mohanan 9066 (CALI). Tamil Nadu, Tirunelveli district, Ambasamudram to Kannikkatti, 31.12.1977, K.M. Matthew 15627; Ibid., 01.01.1978, K.M. Matthew 15796 (RHT); Chittar river-way to Agasthyamalai, 22.08.1963, A.N. Henry 16971; Kannikkatti, 12.06.1899, C.A. Barber 396; Kannikkatti to Kalivayalpil, 31.05.1901, C.A. Barber 3002; Papanasam hills, 1867, R.H. Beddome s.n.; Valayar forest, 11.07.1976, P. Bhargavan 47445; way to Ainthuthalai, 14.10.1989, R. Gopalan 90662; way to Pothgai malai, 16.10.1989, R. Gopalan 91605; way to Kalivarapil, 23.01.1991, R. Gopalan 94658; s.loc., 1877, R.H. Beddome s.n. (MH) ); s.loc., s.d., R.H. Beddome 5008, 5805 (BM digital image).

Conservation status: The species is distributed in 4 districts in southern Kerala and southern Tami Nadu and where seven locations are known. Only five of these fall in protected areas. The Extent of Occurrence (EOO) is calculated to be $c .600 \mathrm{~km}^{2}$ and the Area of Occupancy (AOO) is $28 \mathrm{~km}^{2}$. During field studies, the authors observed a total of only 52 mature plants at four locations. This might indicate that the species occurs with very few individuals in total and should belong in the Endangered category (EN), D (IUCN, 2019).

Notes: Henckelia repens is the only creeping species in South India while the other species, H. burttii D.J.Middleton \& Mich.Möller, with a very similar habit is found in Northeast India (Burtt \& Panigrahi, 1965; Wood, 1974). Henckelia repens has a longer corolla tube (up to 1.5) compared to other South Indian species. Beddome's illustration (Icon. Pl. Ind. Or. 1: 24. t. 120. 1874) and drawings on the type sheet at $\mathrm{K}$ differ with the protologue in two points. The anthers are cohering in the specimens but they are drawn in the icon and on the illustration on the type sheet as non-coherent. Similarly, the filament is broad except at the apex, but it is drawn as narrow along its entire length in the type sheet and broad at the basal region in the icon.
Henckelia wayanadensis Janeesha \& Nampy, sp. nov.

Figs. 22 \& 23a1-i1

Henckelia wayanadensis is morphologically similar to $H$. fischeri, but can be readily identified by its elliptic to widely elliptic leaves ( $v s$. ovate), ovate hispid bracts ( $v s$. lanceolate tomentose bracts), small flowers $12-14 \times 6.7-11 \mathrm{~mm}$ (vs. large flowers $11-17 \times 9-22 \mathrm{~mm}$ ), and purple glands on the ovary ( $v s$. hyaline glandular hairs on the ovary).

Type: INDIA, Kerala, Wayanad district, Kaduvakuzhi hill top, 1200 m, 08.07.2016, A.P. Janeesha \& Santhosh Nampy 137672 (holo CALI!; iso $\mathrm{MH} !)$

Perennials, rosette-forming, herbs with rootstocks. Roots adventitious, thin. Leaves 8-20, basal, usually in whorls of four; petioles $4-11.5 \mathrm{~cm}$ long, pubescent, winged; wings 3-8 mm broad; laminae elliptic to widely elliptic, $8-19.2 \times 6-12 \mathrm{~cm}$, obtuse to slightly acute at apex, attenuate at base, margins doubly crenate, ultimate segments irregular, first crenation very deep, surfaces usually rugose, white tomentose on both surfaces, becoming less so with age; veins 6-8 on each side, much branched and conspicuous beneath. Cymes axillary, 4-10 per plant, 3-5 times divided, 35-63-flowered; peduncles terete, $23-30 \mathrm{~cm}$ long, green-pale reddish brown, tomentose; bracts two at each branching point, ovate, 5.5-6.6 × 1-2 mm, slightly acute at apex, margin entire, hispid, green; pedicels terete, 4-26 mm long, narrow, tomentose. Flowers 12-14 × 6.7-11 mm, held horizontal. Calyx 5partite, very deeply divided or basally connate; lobes linear to ovate-elliptic, unequal, posterior lobe small, 2.7-3.4 × 0.5-0.9 mm, others 3.55-4.35 × 0.8-1.4 mm, green, obtuse at apex, margins entire, glandular hairy. Corolla zygomorphic, ventricose, two lipped, glandular hairy outside, glabrous inside; tube 5-9 × 3.4-4.5 mm, dark to pale lilac with a yellow blotch at the throat, swollen towards the mouth; lobes widely ovate, rounded at apex dark to pale lilac, upper lip of two lobes, 3.7-4 × 4-5.1 $\mathrm{mm}$, lower lip of three lobes, 3.9-4.4 × 4.2-5.8 mm. Stamens 2, adnate to the base of corolla, included; filaments $3.3-4.3 \mathrm{~mm}$ long, glabrous, yellow; anthers reniform, 1.2-1.7 × 0.4-0.76 mm, milky 

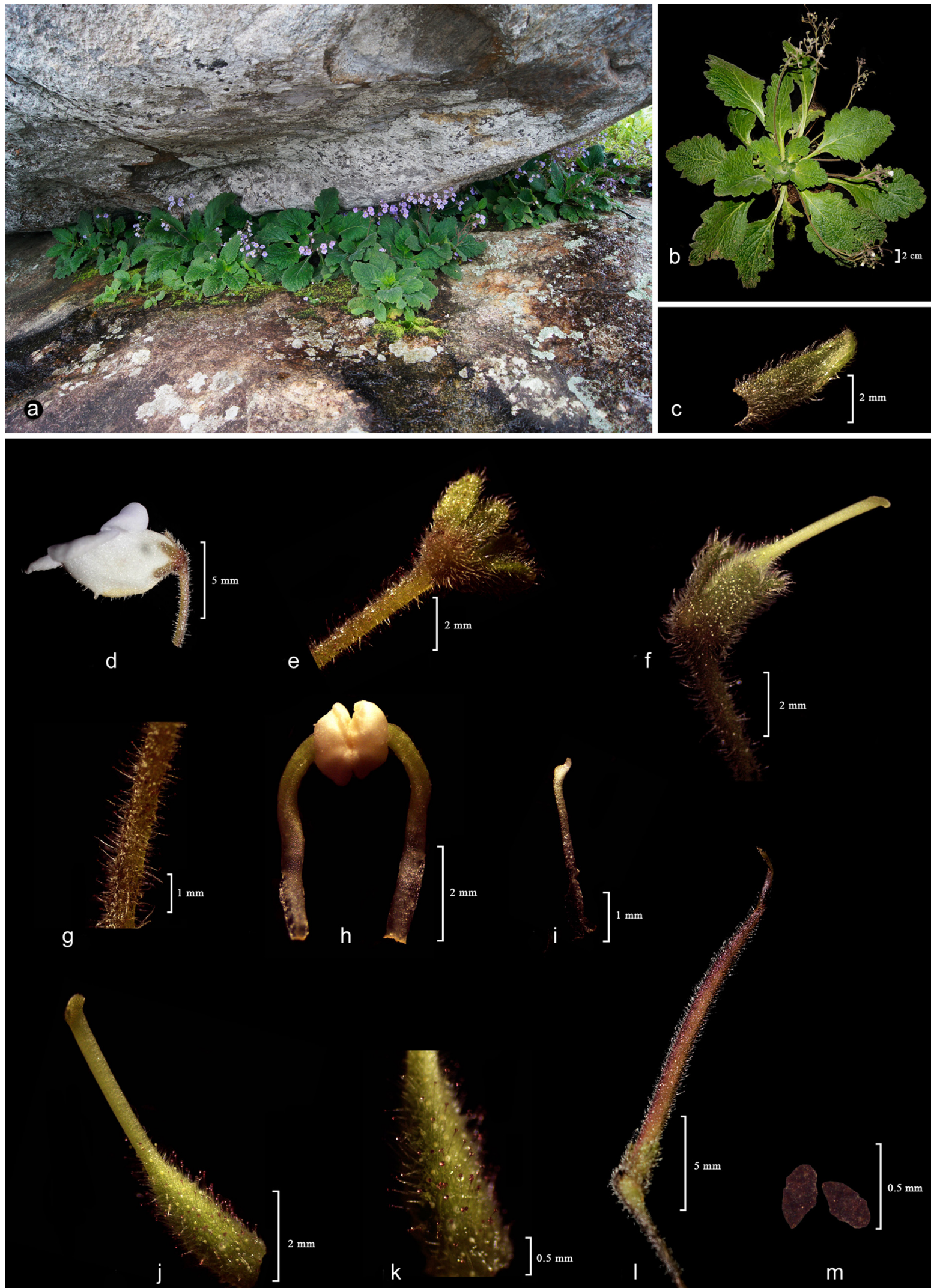

Fig. 22. Henckelia wayanadensis Janeesha \& Nampy: a. Plants in natural habitat; b. Habit; c. Bract; d. Flower; e. Calyx; f. Calyx with pistil; g. Hairs on the pedicel; h. Stamens; i. Staminode; j. Pistil; k. Purplish glandular hairs on the ovary; I. Immature capsule; m. Seeds (a-I from A.P. Janeesha \& P.G. Arunkumar 137671; m from A.P. Janeesha \& K. Haseem 137690). 

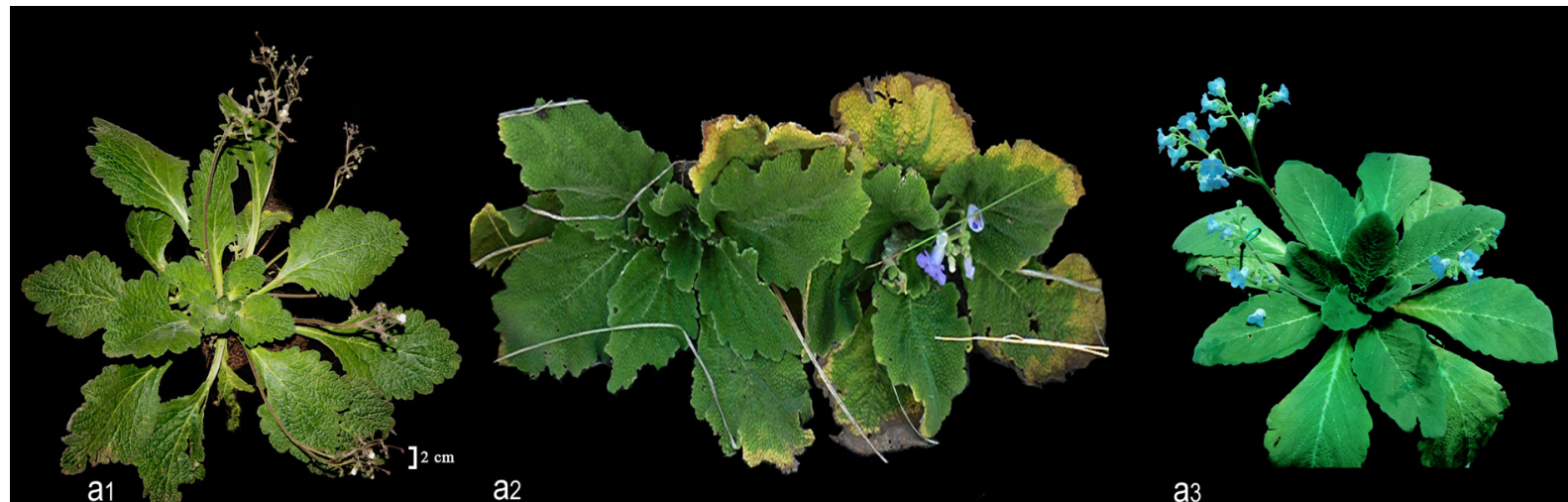

a3
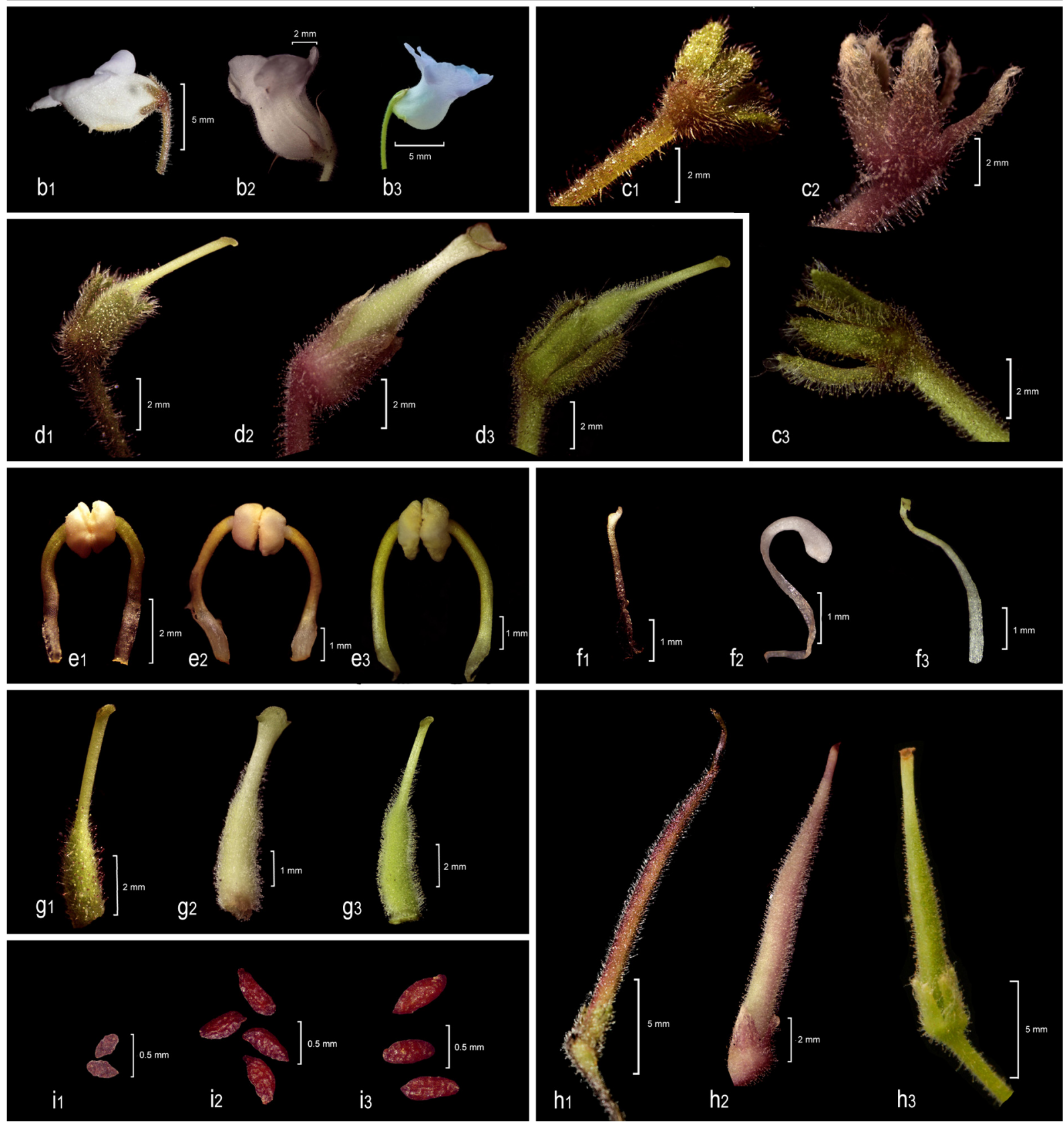

Fig. 23. Comparison of Henckelia wayanadensis Janeesha \& Nampy and H. fischeri (Gamble) A.Weber \& B.L.Burtt: a1-i1 H. wayanadensis; a2-i2 \& a3-i3 H. fischeri: a. Habit; b. Flowers; c. Calyx; d. Calyx with pistil; e. Stamens; f. Staminodes; g. Pistil; h. Immature capsules; i. Seeds. 
white to cream. Staminodes 2; filaments $1.3-3 \mathrm{~mm}$ long, hyaline, glabrous; antherodes a small knoblike structure. Ovary ovoid to oblong, 2.7-3.5 × 0.9-1.3 mm, green, glandular hairy, gland tip purplish; style terete, 2.2-3.6 mm long, green, glabrous towards apex, glandular hairy towards base; stigma obliquely subcapitate, $0.4-0.5 \times 0.22-$ $0.24 \mathrm{~mm}$, papillate, pale green. Capsules cylindrical, straight or slightly curved, 20-22 × 1.6-2 mm, plagiocarpic, dehiscing loculicidally long the dorsal side, reddish brown, glandular, hispid, with persistent stigma and calyx. Seeds numerous, elliptic, $0.65-0.72 \times 0.20-0.24 \mathrm{~mm}$, truncate at both; testa dark brown, reticulate.

Flowering \& fruiting: Flowering from July to August and fruiting from August to September.

Etymology: The species epithet 'wayanadensis' refers to the locality 'Wayanad', the floristically rich district of Kerala in which the new taxon was discovered.

Habitat: In rock crevices, usually at an elevation above $900 \mathrm{~m}$, in association with Parahemionitis sp. (Pteridaceae), Cyanotis sp. (Commelinaceae) and some moss and grass species.

Distribution: Hitherto known only from the type locality (Fig. 26).

Specimens examined: INDIA, Kerala, Wayanad district, Kaduvakuzhi hill top, 08.07.2016, A.P. Janeesha \& P.G. Arunkumar 137671; Ibid., 15.09.2016, A.P. Janeesha \& K. Haseem 137690; on the way to Kaduvakuzhi, $500 \mathrm{~m}$ away from Adivaram, 08.07.2016, A.P. Janeesha \& P.G. Arunkumar 137670 (CALI).

Conservation status: The new species is currently known only from one locality with three subpopulations in an unprotected area, separated by a distance of 50-60 meters, with a total of 61 mature plants. There is the possibility of disturbances in the future due to human activities, where local peoples are exploring the area for wood, forage grasses etc. The Area of Occupancy (AOO) is likely to be less than $10 \mathrm{~km}^{2}$. Therefore the species is categorized as Critically endangered (CR),
B2ab(iii,iv,v) according to IUCN criteria (IUCN, 2019).

Henckelia wightii (C.B.Clarke) A.Weber \& B.L.Burtt, Beitr. Biol. Pflanzen 70: 359. 1998[“1997"]. Didymocarpus rottleriana Wall., Wight, Icon. Pl. Ind. Orient. 4: 24. t. 1348. 1848. Didymocarpus rottlerianus Wall. ex R.Br. var. wightii C.B.Clarke in A.DC. \& C.DC., Monogr. Phan. 5: 101. 1883, C.B.Clarke in Hook.f., Fl. Brit. India 4: 354. 1885["1884"]. Didymocarpus wightii (C.B.Clarke) Gamble, Fl. Madras 2: 989. 1924; A.N.Henry et al., Fl. Tamil Nadu Ind., Ser I: Analysis 2: 133. 1987; M.Ahmedullah \& M.P.Nayar, Endemic Pl. Indian Region 1: 143. 1987; Sasidh., Biodivers. Doc. Kerala Part 6: Fl. Pl. 332. 2004; T.S.Nayar et al., Fl. Pl. Kerala 343. 2006, Fl. Pl. Western Ghats 1: 529. 2014. Lectotype (designated here): INDIA, Tamil Nadu, Tirunelveli district, Shevghery, Courtallam, s.d., Wight 2323 (K [K000858201 digital image!]; isolecto: E [E00155179 digital image!]).

Didymocarpus sivagiriensis Rajakumar, Selvak., S.Murug. \& Chellap., Indian J. Forest. 32(3): 481. 2009; T.S.Nayar et al., Fl. Pl. Western Ghats 1: 527. 2014, syn. nov. Henckelia sivagiriensis (Rajakumar, Selvak., S.Murug. \& Chellap.) E.S.S. Kumar, Polish Bot. J. 59(1): 149. 2014. Type: INDIA, Tamil Nadu, Tenkasi district, Thalaiyanai reserve forest, s.d., Rajakumar, Selvakumari, Murugesan $\mathcal{E}$ Chellapperumal 829 (holo JCH).

Figs. $24 \& 25$

Perennials, rosette-forming, hairy herbs with rootstocks. Roots adventitious, thick. Leaves 1220 , basal, in whorls of $4-7$, sessile to sub-sessile; laminae spatulate-obovate to elliptic, 3.8-10.7 × $1.6-3.6 \mathrm{~cm}$, obtuse to rounded at apex, attenuate at base, margins slightly crenate and undulate, surfaces smooth, velvety, with white woolly hairs on both surfaces, but more so on the younger leaves and on lower veins, becoming less so with age; veins 4-6 on each side, much branched and conspicuous beneath. Cymes axillary, dense; 2-6 per plant, 3-4 times divided, 5-13-flowered; peduncles terete, 10$18 \mathrm{~cm}$ long, greenish, glandular hairy; bracts two at each branching point, minute, completely 

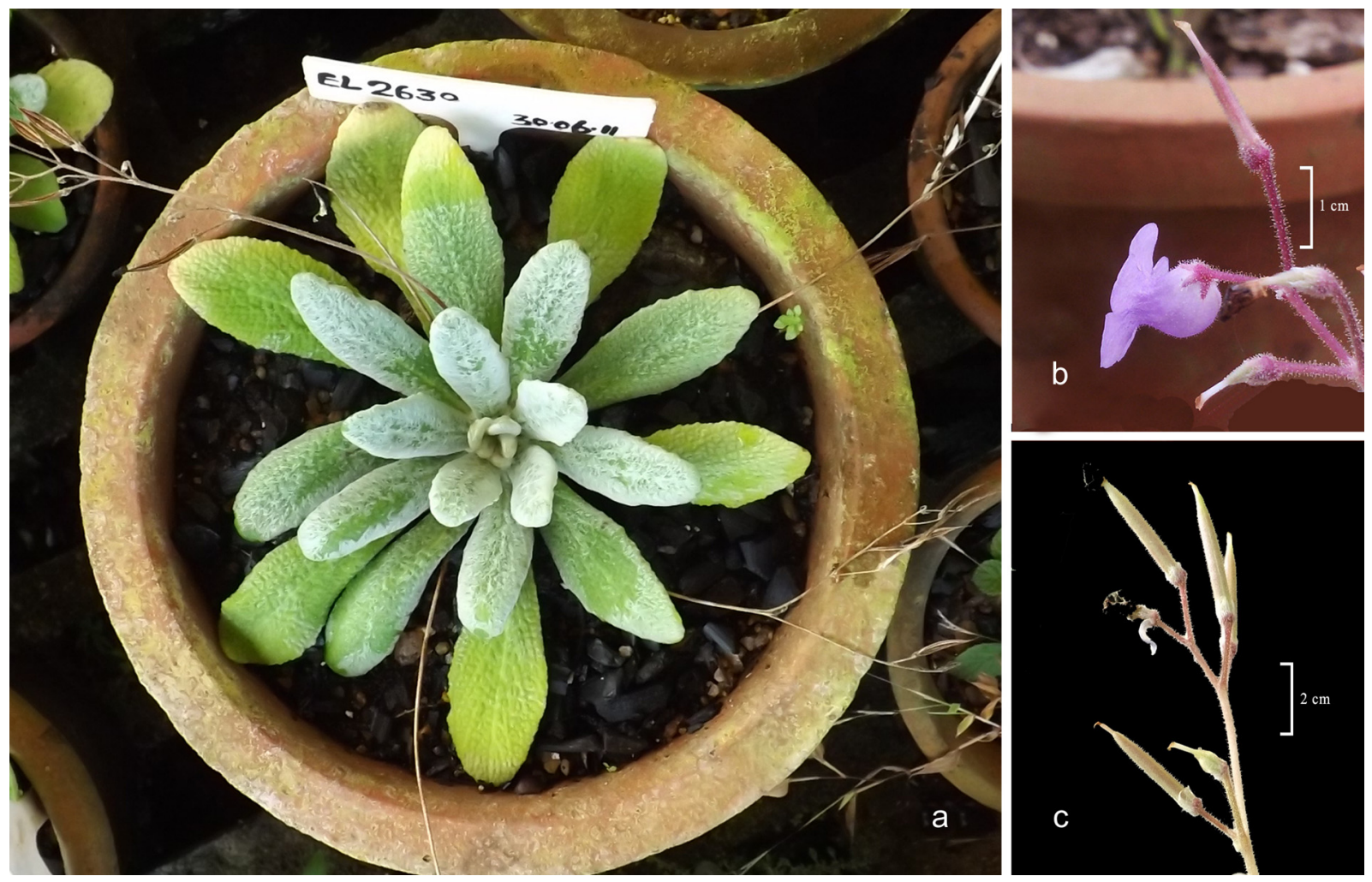

Fig. 24. Henckelia wightii (C.B.Clarke) A.Weber \& B.L.Burtt: a. Habit showing whorls of five leaves; b. Flower; c. Immature capsules (from plants cultivated at Gurukula Botanical Sanctuary, Periya).

woolly, c. $2 \mathrm{~mm}$ long; pedicels terete, 9-18 $\mathrm{mm}$ long, narrow, glandular hairy. Flowers 11-15 × 10$14 \mathrm{~mm}$. Calyx 5-partite, very deeply divided; lobes oblong-lanceolate, unequal, posterior lobe small, $1.8-2.1 \times 0.45-0.6 \mathrm{~mm}$, other lobes, $2-3 \times 0.6-0.8$ $\mathrm{mm}$, slightly acute at apex, margins entire, with white woolly hairs on the upper side. Corolla zygomorphic, ventricose, two lipped, glandular hairy outside, glabrous inside; tube lilac to pale lilac with a yellow patch in the throat; lobes orbicular, ovate to rounded at apex, lilac to pale lilac, upper lip of two lobes, lower lip of three lobes. Stamens 2 , adnate to the base of corolla, included; filaments c. $2 \mathrm{~mm}$ long, glabrous; anthers reniform, glabrous. Staminodes 3 (sometimes 2); filaments c. $1.2 \mathrm{~mm}$ long, glabrous; antherodes not seen. Ovary ovoid to cylindrical, c. $2.5 \mathrm{~mm}$ long, covered with dense white woolly hairs, completely enclosed within calyx; style terete, c. $1.3 \mathrm{~mm}$ long, glabrous; stigma obliquely subcapitate, papillate, pale green. Capsules linear-cylindrical, straight, 11-17 mm long, plagiocarpic, dehiscing loculicidally along the

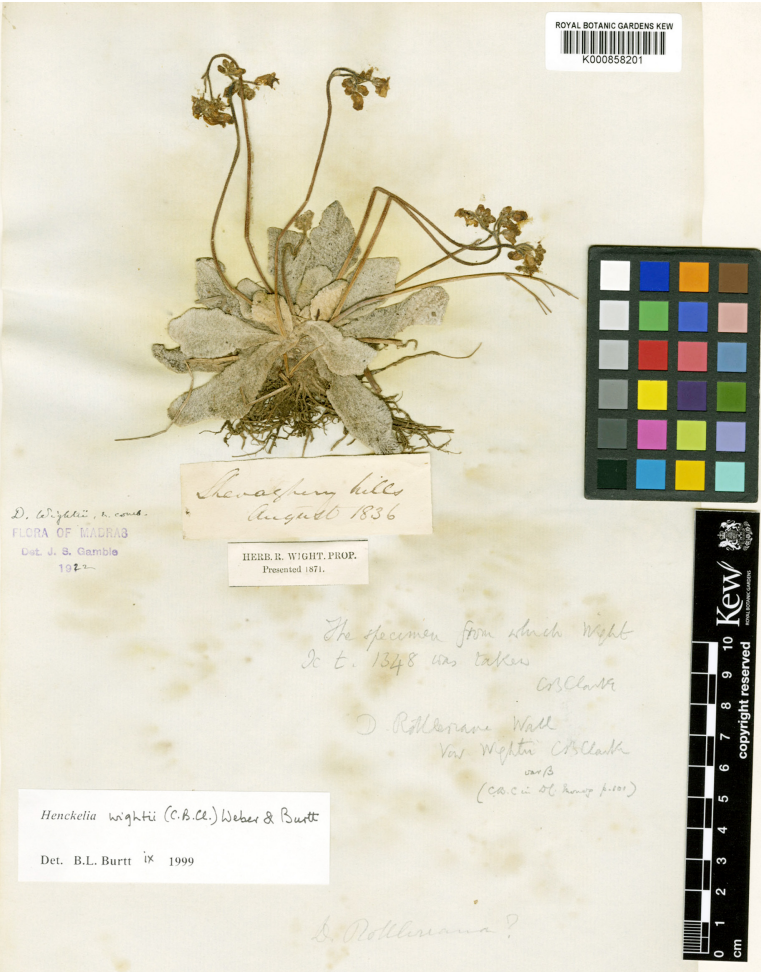

Fig. 25. Lectotype of Henckelia wightii (C.B.Clarke) A.Weber \& B.L.Burtt. (C) The Board of Trustees for The Royal Botanic Gardens, Kew. Reproduced with permission. 


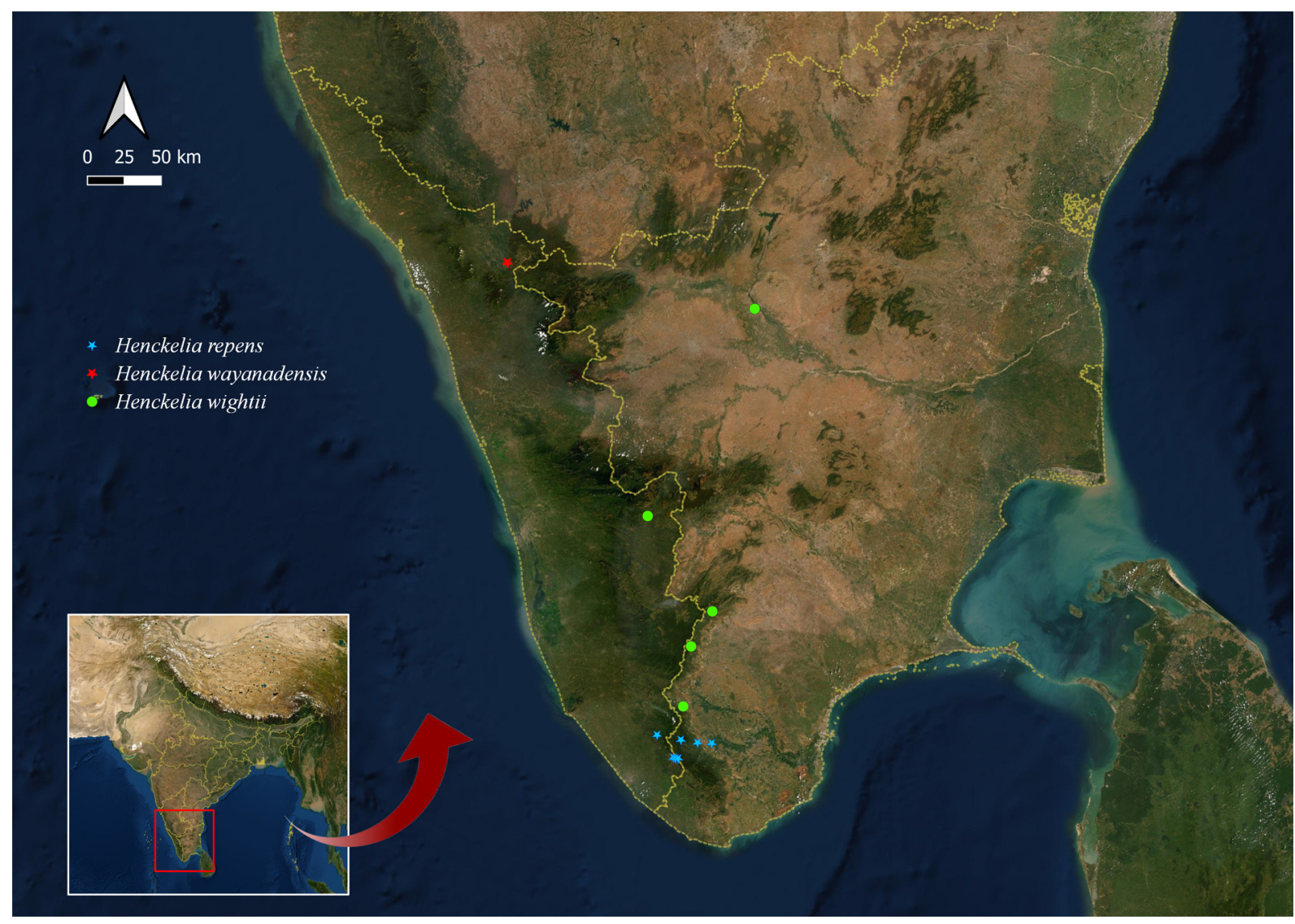

Fig. 26. Distribution of Henckelia repens (Bedd.) A.Weber \& B.L.Burtt, H. wayanadensis Janeesha \& Nampy and H. wightii (C.B.Clarke) A.Weber \& B.L.Burtt in South India (drawn using QGIS v.3.12.1).

dorsal side, green, tomentulose with persistent stigma and calyx. Seeds numerous, reticulate.

Flowering \& fruiting: Flowering from July to October and fruiting from August to November.

Habitat: On moist rocks.

Distribution: Endemic to the southern Western Ghats (Fig. 26).

Specimens examined: INDIA, Kerala, Idukki district, Karadippara-Munnar, 25.07.2013, A.P. Janeesha \& K.M. Manudev 134203 (CALI). Tamil Nadu, Erode district, Attamalai hills, 24.07.1988, N. Venkatasubramanian 1424 (FRC); Tenkasi district, New Courtallam, 16.07.2015, A.P. Janeesha, S. Resmi \& P.G. Arunkumar 137628 (CALI); Virudhunagar district, Kendiparai to Cumbumedu-Ayyanarkovil, 23.09.1971, E. Vajravelu 38720; Mudaliarattu, 11.12.1971, E. Vajravelu 39339 (MH).
Conservation status: The species is known from four districts in Kerala and Tamil Nadu where a total of five localities were known. The Extent of Occurrence (EOO) is estimated to be c. $2100 \mathrm{~km}^{2}$ and Area of Occupancy (AOO) is $20 \mathrm{~km}^{2}$. During a period between 2013 and 2017, one of the authors (JAP) visited four localities except Ayyanarkovil and was unable to observe more than 5 mature plants in two localities, i.e., Munnar and Courtallam. Thus, a decline in number of localities from five to two over five years has been observed, in (1) EOO (ii) AOO; (iv) number of locations or subpopulations and (v) number of mature individuals. Because of the very low number of plants found, we consider this species to be Critically endangered (CR), D according to IUCN guidelines, with a high risk of extinction in the near future (IUCN, 2019).

Notes: Rajakumar et al. (2009) described Henckelia sivagiriensis (as Didymocarpus sivagiriensis Rajkumar, 
Semvak., S.Murug. \& Chellap.) and differentiated it from H. wightii by its longer leaves, presence of glandular hairs, longer dichotomously branched scapes, bluish corolla, puberulous style and longer capsules. Critical examination of specimens and protologues of both these taxa revealed that the characters outlined for separating the former from the latter are intergrading (see description above) and hence $H$. sivagiriensis is reduced to synonymy of $H$. wightii.

Typification: Wight described this species based on material collected from the Shevghery hills, Courtallam in Tamil Nadu. Since Vitek et al. (2000) cited only syntypes, it cannot be considered a lectotypification. Two sheets of Wight's materials were located, while searching for the type specimens, one each in E (E00155179) and K (K000858201). The sheet K000858201 has an HRWP label and bears a field label, "Shevghery hills August 1836" and an annotation by Clarke "the specimen from which Wight Ic. 1348 was taken". The sheet itself has the no. 2323/1837 in the bottom left hand corner. The sheet in E (E00155179) has an HWP label annotated "Didymocarpus rottlerianus var. wightii" and "Herb. Wight. propr. n. 2679" in the top right hand corner and another in the lower left hand corner " 1837 , No 2323", presumably referring to the year 1837. The specimen at K (K000858201) used for illustrating this species by Wight is selected here as the lectotype and the other at E (E00155179) as the isolectotype.

\section{Acknowledgements}

The authors are grateful to Curators of BM, CAL, $\mathrm{E}$ and $\mathrm{MH}$ for permitting consultation of the specimens; Dr. J.V. Sudhakar (MH), Dr. Mark Carine (BM), Dr. K. Karthigeyan (CAL) and Dr. Lesley Walsingham $(\mathrm{K})$ for comments on the annotations on the herbarium materials; Dr. Kanchi N. Gandhi (Nomenclature Registrar, Harward University) for Nomenclatural suggestions; Dr. Anton Weber (Vienna) for literature; Dr. A.K. Pradeep (Calicut University), Dr. K.M. Manudev
(St. Joseph's College (Autonomous), Kozhikode; Gurukula Botanical Sanctuary, Periya, Wayanad and Dr. (Sr.) Jaseena Joseph (Principal, Providence Women's College, Kozhikode) for various helps; Dr. Shahina P.M., Mr. Arunkumar P.G., Ms. Resmi S, Mr. Syam Radh S., Dr. Shinoj K., Dr. Thoiba K., Dr. Smitha K., (Research Scholars, Calicut University), Mr. Habeeb Rahman, Mr. Rajeevan and Mr. Ahmadul Kabeer for helping collections. We are thankful to Dr. Michael Möller (E) and Dr. David Middleton (Singapore Botanic Gardens) for their valuable comments for improving the manuscript. We thank Dr. K.M. Prabhukumar (CMPR, Kottakkal) and Dr. M.G. Prasad (NBRI, Lucknow) for photographic images of some species of Henckelia. SN is thankful to SERB New Delhi (EMR/2016/007346 dated 01/11/2018) for funding and JAP to University Grant Commission for Fellowship.

\section{Literature Cited}

BEDDOME R.H. 1874. Cyrtandraceae. In: BEDDOME R.H. (ed.), Icones plantarum Indiae orientalis. Volume 1. Today and Tomorrows Printers and Publishers, New Delhi. pp. 24-39.

BORAH D., TARAM M., JOE A. \& S. NEELAMKAVIL 2019. Henckelia collegii-sancti-thomasii: a new species of Henckelia (Gesneriaceae) from Northeastern India. Phytotaxa 415(4): 247-251. http://dx.doi.org/10.11646/ phytotaxa.415.4.10

BROWN R. 1839. Cyrtandreae. In: BENNETT J.J. \& R.R. BROWN (eds.), Plantae Javanicae Rariores, Descriptae Iconibus Illustratae, quas in Insula Java, Annis 1802-1818. Part 2 (including t.24 from Part1). W.H. Allen, London. p.105-122.

BURTT B.L. 1954. Studies in the Gesneriaceae of the Old World I: general introduction. Notes from the Royal Botanic Garden Edinburgh 21: 185-192.

BURTT B.L. \& G. PANIGRAHI 1965. Studies in the Gesneriaceae of the Old World XXVI: a contribution to the study of Chirita. Notes from the Royal Botanic Garden Edinburgh 26(3): 265-266.

CAI L., LIU D.T., ZHANG P. \& Z.L. DAO 2019. Two new species of Henckelia (Gesneriaceae) from Southeastern Yunnan, China. PhytoKeys 130: 151-160. https://doi.org/10.3897/phytokeys.130.33988 
CANDOLLE A.L.P.P. DE 1845. Cyrtandraceae. In: CANDOLLE A.L.P.P. DE (ed.), Prodromus Systematis Naturalis Regni Vegetabilis, sive enumeratio contracta Ordinum, Generum, Specierunque Plantarum huc usque cognitarum, juxta methodi naturalis normas digesta. Volume 9. Fortin, Masson \& Co., Paris. pp. 258-286.

CLARKE C.B. 1883. Cyrtandreae. In: CANDOLLE A. DE \& C. DE CANDOLlE (eds.), Monographiae Phanerogamarum. G.Masson, Paris. pp. 1-303.

CLARKE C.B. 1885[“1884”]. Gesneriaceae. In: HOOKER J.D. (ed.), The Flora of British India. Volume 4. L.Reeve \& Co., London. pp. 336-375.

FISCHER C.E.C. 1938. Didymocarpus gambleana. Bulletin of Miscellaneous Information, (Royal Gardens, Kew) 1938(1): 36.

GAMBLE J.S. 1924. Flora of the Presidency of Madras, Scrophulariaceae to Plantaginaceae. Volume 2. Bishen Singh and Mahendrapal Singh, Dehra Dun. pp. 983-992.

HENRY A.N., KUMARI G.R. \& V. CHITRA 1987. Flora of Tamil Nadu. Series 1: Analysis, Volume 2. Botanical Survey of India, Kolkata. pp. 132-133.

IUCN STANDARDS AND PETITIONS SUBCOMMITTEE 2019. Guidelines for using the IUCN Red list categories and criteria, Version 13. Available at: http:// www.iucnredlist.org/documents/RedListGuidelines. pdf. (Accessed on 25.03.2020).

JANEESHA A.P. 2017. Taxonomic studies on family Gesneriaceae in South India supplemented with molecular data. Ph.D. Thesis, University of Calicut. pp. 1-314.

JANEESHA A.P. \& S. NAMPY 2015. Henckelia bracteata, a new species of Gesneriaceae from southern Western Ghats, India, and lectotypification of Didymocarpus humboldtianus (H. humboldtiana). Willdenowia 45(1): 5359. https://doi.org/10.3372/wi.45.45105

JANEESHA A.P. \& S. NAMPY 2016. Lectotypification of three names in the genus Henckelia Spreng. (Gesneriaceae). Phytotaxa 268(1): 80-84. http:// dx.doi.org/10.11646/phytotaxa.268.1.6

KANTHRAJ A.S., RANA T.S. \& K.N. NAIR 2020. Henckelia umbellata (Gesneriaceae), a new species from the eastern Himalaya of India. Rheedea this issue. https:/ /dx.doi.org/10.22244/rheedea.2020.30.01.08

KRISHNA G. \& P. LAKSHMINARASIMHAN 2018. A new species of Henckelia (Gesneriaceae) from Arunachal Pradesh, India. Taiwania 63(4): 397-401. https://doi.org/ 10.6165/tai.2018.63.397

KUMAR E.S.S. 2014. A new combination in Henckelia (Gesneriaceae). Polish Botanical Journal 59(1): 149. https:/ /doi.org/10.2478/pbj-2014-0002

KUNTZE, O. 1891. Revisio generum plantarum: vascularium omnium atque multarum secundum leges nomenclaturae internationales cum enumeratione plantarum exoticarum in itinere mundi collectarum. Volume 2. A. Felix, Leipzig. p. 476.

MANUDEV K.M., WEBER A. \& S. NAMPY 2012. Henckelia pradeepiana, a new species of Gesneriaceae from southern Western Ghats, India. Rheedea 22(2): 119-123.

MIDDLETON D.J., WEBER A., YAO T.L., SONTAG S. \& M. MÖLLER 2013. The current status of the species hitherto assigned to Henckelia (Gesneriaceae). Edinburgh Journal of Botany 70: 1-20. http://dx.doi.org/10.1017/ S0960428613000127

MÖLLER M. \& J.L. CLARK 2013. The state of molecular studies in the family Gesneriaceae: a review. Selbyana 31(2): 95-125.

MÖLLER M., NAMPY S., JANEESHA A.P. \& A. WEBER 2017. The Gesneriaceae of India: consequences of updated generic concepts and new family classification. Rheedea 27(1): 23-41. https://dx.doi.org/10.22244/ rheedea.2017.27.1.5

NAYAR P.H. \& A.R.K. SASTRY 1990. Red data book of Indian plants, Volume 3. Botanical Survey of India, Kolkata. p. 157.

QGIS DEVELOPMENT TEAM 2020. QGIS Geographic Information System. QGIS V.3.12.1-Bucuresti. Open Source Geospatial Foundation Project. Available at: https://qgis.osgeo.org

RAJAKUMAR T.J.S., SELVAKUMARI R., MURUGESAN S. \& N. CHELLAPERUMAL 2009. Didymocarpus sivagiriensis, a new species of Gesneriaceae from Tirunelveli district, Tamil Nadu, India. Indian Journal of Forestry 32(3): 481-483.

RAMASWAMY S.V. 1914. Didymocarpus meeboldii. Records of the Botanical Survey of India 6: 43.

RANASINGHE S., MIDDLETON D.J., ATKINS H.J., MILNE R. \& M. MÖLLER 2019. Typification of Sri Lankan Gesneriaceae. Edinburgh Journal of Botany 76(2): 221-229. https://doi.org/10.1017/S0960428619000027

RANASINGHE S., MILNE R., JAYASEKARA R., RUBASINGHE S. \& M. MÖLLER 2016. Henckelia wijesundarae (Gesneriaceae), a new endemic species from Sri Lanka, and lectotypification of Chirita walkerae and C. walkerae var. parviflora. Willdenowia 46(2): 213-224. https://doi.org/10.3372/wi.46.46202

RATTER J.A. \& H.T. PRENTICE 1967. Chromosome numbers in the Gesneriaceae III. Notes from the Royal Botanic Garden Edinburgh 27: 205-209.

SIMPSON M.G. 2006. Plant Systematics, First edition. Elsevier Academic Press, Burlington, USA. pp. 345-540.

SINHA B.K. \& S. DATTA 2016. Taxonomic account of the family Gesneriaceae in Northeast India. Nelumbo 58: 1-43. https://doi.org/10.20324/nelumbo/v58/2016/ 105932 
SIRIMONGKOL S., PARNELL J. A.N., HODKINSON T.R., MIDDLETON D.J. \& C. PUGLISI 2019. Five new species of Henckelia (Gesneriaceae) from Myanmar and Thailand. Thai Forest Bulletin, Botany 47(1): 38-54. https://doi.org/10.20531/tfb.2019.47.1.08

SPRENGEL K. 1817. Anleitung zur Kenntniss der Gewächse, Second edition, Volume 2(1). C.A. Kümmel, Halle. p. 502.

STAFLEU F.A. \& R.S. COWAN 1976. Taxonomic Literature: A selective guide to botanical publications and collections with dates, commentaries and types, Second edition, Volume 1. A-G. Regnum Vegetabile 94. Bohn, Scheltema \& Holkema, Utrecht. pp. 162-164. https:// doi.org/10.5962/bhl.title.48631

STAFLEU F.A. \& R.S. COWAN 1983. Taxonomic Literature: A selective guide to botanical publications and collections with dates, commentaries and types, Second edition, Volume 4. P-Sak. Regnum Vegetabile 110. Bohn, Scheltema \& Holkema, Utrecht. p. 933. https:// doi.org/10.5962/bhl.title.48631

STAFLEU F.A. \& R.S. COWAN 1988. Taxonomic Literature: A selective guide to botanical publications and collections with dates, commentaries and types, Second edition, Volume 7. W.Z. Regnum Vegetabile 116. Bohn, Scheltema \& Holkema, Utrecht. pp. 37-41. https:/ /doi.org/10.5962/bhl.title.48631

THATHACHAR T. 1942. Studies in Gesneriaceae, gametogenesis and embryogeny of Didymocarpus tomentosa Wight. Journal of the Indian Botanical Society 21: 185-193.

THIERS B. (continuously updated). Index Herbariorum: A global directory of public Herbaria and associated staff. New York Botanical Garden's Virtual Herbarium. Available at: http://sweetgum.nybg.org/science/ih (Accessed on 17.11.2019).

TURLAND N.J., WIERSEMA J.H., BARRIE F.R., GREUTER W., HAWKSWORTH D.L., HERENDEEN P.S., KNAPP S., KUSBER W.H., LI D.Z., MARHOLD K., MAY T.W., M NEILL J., MONRO A.M., PRADO J., PRICE M.J. \& G.F. SMITH (eds.) 2018. International Code of Nomenclature for Algae, Fungi, and Plants (Shenzhen Code) Adopted by the Nineteenth International Botanical Congress Shenzhen, China, July 2017.
Regnum Vegetabile 159, Volume 38. Glashütten, Koeltz Botanical Books, Glashütten. pp. 1-254. https://doi.org/ 10.12705/Code. 2018

VAHL M.H. 1804. Enumeratio Plantarum: vel abalii, vela $b$ ipso observatarum, cum earum differeniüs specificis, synonymis selectis et descriptionbus succinctis, Volume 1. J.H. Schubothe, Havniae. p. 88.

VITEK E., WEBER A. \& B.L. BURTT 2000. Names, types and current placement of the species hitherto referred to Didymocarpus, Loxocarpus, Codonoboea, Platyadenia and Henckelia (Gesneriaceae). Annalen des Naturhistorischen Museums in Wien 102B: 477-530.

WALLICH N. 1819. Art. XXVI: Notice of the progress of Botanical Science in Bengal, being the substance of a letter from Dr. Wallich, superintendent of the Botanical Garden near Culcutta, to Francis Hamilton. Edinburgh Philosophical Journal 1: 377-380.

WALLICH N. 1829. A numerical list of dried specimens of plants in the East India Company's Museum collected under the superintendence of Dr. Wallich of the Company's botanic garden at Calcutta. London.

WALTER K.S. \& H.J. GILLET 1997. Red list of threatened plants. The World Conservation Union, Gland. UK. p. 291.

WEBER A. \& B.L. BURTT 1998[“1997”]. Remodelling of Didymocarpus and associated genera (Gesneriaceae). Beiträge zur Biologie der Pflanzen 70: 293-363.

WEBER A., MIDDLETON D.J., FORREST A.L., KIEW R., LIM C.L., RAFIDAH A., YAO T.L., SONTAG S., TRIBOUN P., WEI Y.G. \& M. MÖLLER 2011. Molecular systematic and remodelling of Chirita and associated genera (Gesneriaceae). Taxon 60(3): 767-790. https://doi.org/10.1002/tax.603012

WIGHT R. 1848. Icones plantarum Indiae orientalis. Volume 4(2). J.R.I. Wood, Madras. p. 10.

WOOD D. 1974. A revision of Chirita (Gesneriaceae). Notes from the Royal Botanic Garden Edinburgh 33(1): 123-205.

YANG B., DING H.B., FU K.C., YUAN Y.K., YANG H.Y., LI J.W., ZHANG L.X. \& Y.H. TAN 2019. Four new species of Gesneriaceae from Yunnan, Southwest China. PhytoKeys 130: 183-203. https://doi.org/10.3897/ phytokeys.130.34001 